\title{
Slender Ca II H fibrils observed by SUNRISE/SuFI
}

\author{
Dissertation \\ zur Erlangung des mathematisch-naturwissenschaftlichen Doktorgrades \\ "Doctor rerum naturalium" \\ der Georg-August-Universität Göttingen \\ im Promotionsprogramm PROPHYS \\ der Georg-August University School of Science (GAUSS)
}

\author{
vorgelegt von \\ Ricardo Jorge Maranhas Gafeira \\ aus Melo, Portugal
}

Göttingen, 2017 
Betreuungsausschuss

Prof. Dr. Sami K. Solanki

Max-Planck-Institut für Sonnensystemforschung, Göttingen, Germany

Prof. Dr. Laurent Gizon

Institut für Astrophysik, Georg-August-Universität Göttingen, Germany

Dr. Andreas Lagg

Max-Planck-Institut für Sonnensystemforschung, Göttingen, Germany

Mitglieder der Prüfungskommision

Referent: Prof. Dr. Sami K. Solanki

Max-Planck-Institut für Sonnensystemforschung, Göttingen, Germany

Korreferent: Prof. Dr. Laurent Gizon

Institut für Astrophysik, Georg-August-Universität Göttingen, Germany

Weitere Mitglieder der Prüfungskommission:

Prof. Dr. Hardi Peter

Max-Planck-Institut für Sonnensystemforschung, Göttingen, Germany

Prof. Dr. Jean Niemeyer

Institut für Astrophysik, Georg-August-Universität Göttingen, Germany

Prof. Dr. Wolfram Kollatschny

Institut für Astrophysik, Georg-August-Universität Göttingen, Germany

Prof. Dr. Ariane Frey

Institut für Astrophysik, Georg-August-Universität Göttingen, Germany

Tag der mündlichen Prüfung: 31. Januar 2018 


\section{Contents}

$\begin{array}{ll}\text { Summary } & 5\end{array}$

$\begin{array}{ll}\text { Zusammenfassung } & 7\end{array}$

1 Introduction 9

1.1 Connection between the photosphere and

1.2 Chromospheric jets and fibril like structures . . . . . . . . . . . 15

1.2.1 General properties of small scale chromospheric jet and fibril-like structures . . . . . . . . . . . . . . 16

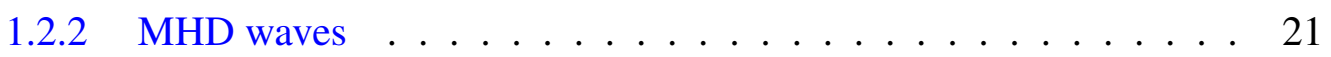

1.3 The SunRISE Observatory . . . . . . . . . . . . . . . . . . . . . 24

1.3.1 SUNRISE Filter Imager (SUFI) . . . . . . . . . . . . . . 27

1.3 .2 Data reduction . . . . . . . . . . . . . . 28

2 Paper I- Morphological properties of Ca II H slender fibrils 31

2.1 Abstract . . . . . . . . . . . . . . . . . . 31

2.2 Introduction . . . . . . . . . . . . . . . 31

2.3 Data . . . . . . . . . . . . . . . . . . 32

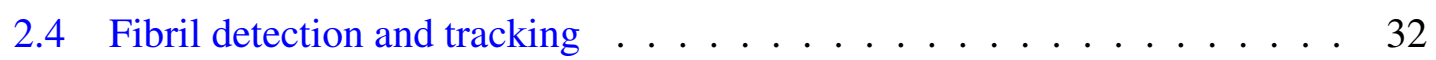

2.5 Fibril morphology . . . . . . . . . . . . . . . . . . . . . . . . . . . . . . 35

2.5 .1 Lifetime . . . . . . . . . . . . . 35

2.5 .2 Width . . . . . . . . . . . . . . . 39

2.5 .3 Length . . . . . . . . . . . . . . . . . . . . . . . . . . . . .

2.5 .4 Curvature . . . . . . . . . . . . . . . . 42

2.5 .5 Brightness variation $\ldots \ldots \ldots \ldots . \ldots . \ldots 42$

2.6 Discussion and conclusions . . . . . . . . . . . . . . . . 47

3 Paper II- Oscillations on width and intensity of slender Ca II H fibrils 51

3.1 Abstract ............................. 51

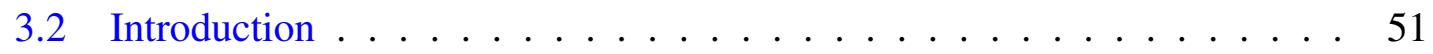

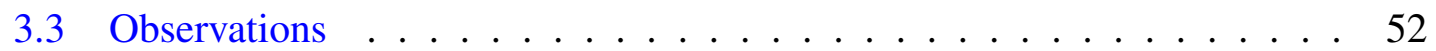

3.4 Analysis and results . . . . . . . . . . . . . . . . . 54

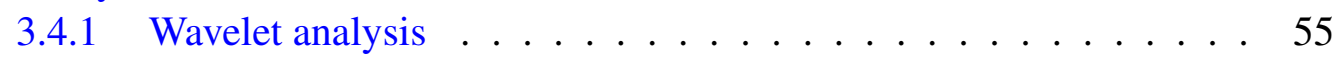

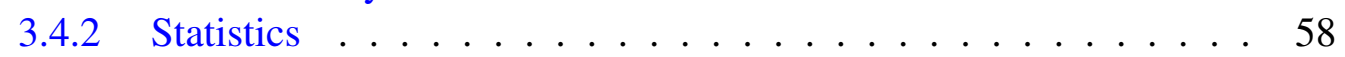

3.5 Discussion and conclusions . . . . . . . . . . . . . . . . 60 
4 Conclusion and Outlook $\quad 63$

$\begin{array}{ll}\text { Bibliography } & 67\end{array}$

A Transverse Oscillations in Slender Ca II H Fibrils Observed with SUNRISE/SUFI 81

A.1 Introduction . . . . . . . . . . . . . . . 81

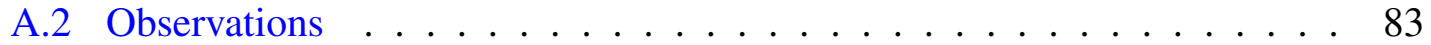

A.3 Analysis and Results . . . . . . . . . . . . . . . . . . . 84

A.3.1 Image Restoration and Fibril Identification . . . . . . . . . . . . . 85

A.3.2 Transverse Oscillations . . . . . . . . . . . . . . . . . . 87

A.3.3 Wave Analysis . . . . . . . . . . . . . . . . 89

A.3.4 Statistics .................... 93

A.3.5 Energy Budget . . . . . . . . . . . . . . . . 96

A.4 Discussion and Conclusions . . . . . . . . . . . . . . . . 97

$\begin{array}{ll}\text { Publications } & 103\end{array}$

$\begin{array}{lr}\text { Acknowledgements } & 105\end{array}$

$\begin{array}{ll}\text { Curriculum vitae } & 107\end{array}$ 


\section{Summary}

This thesis is devoted to analyzing the morphological and oscillatory properties of slender $\mathrm{Ca}$ II H fibrils (SCFs) in the lower solar chromosphere. The aim of this study is to deepen our understanding of the physical nature of these structures and their relevance for the energetics in the solar chromosphere and the layers above.

For this purpose, we use seeing-free high spatial and temporal resolution data in the line core of the $\mathrm{CaIIH}$ line, which is formed in the chromosphere. A time series of narrow-band images in this line of almost 1 hour was obtained during the second science flight of the the SUNRISE observatory by the SUNRISE Filter Imager (SUFI).

In the first part of the thesis we identify and track 598 elongated bright structures (fibrils), observed in the Ca II $\mathrm{H}$ intensity images, in order to estimate their morphological properties. We obtain an average width of around $180 \mathrm{~km}$, a length between 500 and $4000 \mathrm{~km}$, an average lifetime of $\approx 400 \mathrm{~s}$, an average curvature of $0.002 \operatorname{arcsec}^{-1}$, and a maximum lifetime of $\approx 2000 \mathrm{~s}$. We identify two different types of brightness evolution in the SCFs. In one type the intensity increases starting from the center and moving to the edges, and in the other type the increase in intensity first becomes visible near one or both of the ends and expands from there toward the center of the fibril. The results from these studies allow us to compare some of the properties of the SCFs with other chromospheric structures such as spicules of type I and II, or Ca II K fibrils.

The second part of this work is dedicated to the study of the oscillatory behavior of SCFs using a wavelet analysis. One analysis focuses on the oscillations in width and intensity. These parameters are measured at several positions along the fibrils and their periods and phase speeds are analyzed. The width and intensity oscillations show overlapping distributions with median values for their periods of $32 \pm 17 \mathrm{~s}$ and $36 \pm 25 \mathrm{~s}$, respectively. For the phase speeds of these perturbations we obtain values of $11_{-11}^{+49} \mathrm{~km} \mathrm{~s}^{-1}$ for width and $15_{-15}^{+34} \mathrm{~km} \mathrm{~s}^{-1}$ for the intensity. By computing the cross-power spectrum between the two oscillations we observe that in most of the cases they show either an anti-phase or in-phase behavior, with the anti-phase case being much more frequent. This indicates that both oscillations are probably caused by the same wave mode. By comparing the oscillatory behavior and the local sound and Alfvén speeds, we conclude that the most likely wave mode to explain all measurements is the fast sausage mode. A few cases are also compatible with slow sausage mode waves.

Transverse motions of the SCFs were also studied. We obtained median amplitudes and periods on the order of $2.4 \pm 0.8 \mathrm{~km} \mathrm{~s}^{-1}$ and $83 \pm 29 \mathrm{~s}$, respectively. We find that the transverse waves usually propagate along the SCFs with a median phase speed of $9 \pm 14 \mathrm{~km} \mathrm{~s}^{-1}$. In some cases waves propagating in both directions, and also standing waves are observed. This oscillatory behavior can be then described by the so-called kink 
waves that, for the computed wave parameters, can carry an energy flux of $\approx 15 \mathrm{~kW} \mathrm{~m}^{-2}$, which is sufficiently high to heat the chromosphere. 


\section{Zusammenfassung}

Diese Arbeit widmet sich der Analyse der morphologischen Eigenschaften und der Oszillationen sogenannter slender $\mathrm{Ca}$ II $H$ fibrils (SCFs) in den niedrigen Schichten der Chromosphäre der Sonne. Das Ziel dieser Studie ist es, das Verständnis über die physikalische Natur dieser Strukturen zu vertiefen und deren Bedeutung für die Energetik der Chromosphäre und den darüberliegenden Schichten zu ergründen.

$\mathrm{Zu}$ diesem Zweck verwenden wir Daten aufgenommen im Kern der Ca II H Linie, die in der unteren Chromosphäre gebildet wird. Diese Daten sind frei von atmosphärischen Störungen und zeichnen sich durch höchste räumliche und zeitliche Auflösung aus. Eine fast einstündige Zeitserie schmalbandiger Aufnahmen in dieser Linie konnte während des zweiten Fluges des SUNRISE Observatoriums mit dem SUNRISE Filter Imager (SUFI) gewonnen werden.

Im ersten Teil dieser Arbeit werden 598 langgezogene, helle Strukturen (Fibrilen) in den $\mathrm{Ca}$ II H Intensitätsbildern identifiziert und zeitlich verfolgt, um deren morphologischen Eigenschaften zu bestimmen. Dabei erhalten wir eine für die Breite dieser Strukturen einen Mittelwert von $180 \mathrm{~km}$, eine durchschnittliche Lebensdauer von $\approx 400 \mathrm{~s}$, eine durchschnittliche Krümmung von $0.002 \mathrm{arcsec}^{-1}$, und eine maximale Lebensdauer von $\approx 2000$ s. Zwei unterschiedliche Arten von Helligkeitsvariationen in diesen SCFs wurden gefunden. In einem Fall steigt die Intensität in den Fibrilen zuerst in deren Zentrum und breitet sich dann entlang des Fibrils aus, im anderen Fall beginnt die Helligkeitssteigerung in einem oder beiden Fußpunkten und wandert dann zum Zentrum des Fibrils. Die Resultate dieser Studie erlauben eine Gegenüberstellung der Eigenschaften von SCFs mit anderen chromosphärischen Strukturen, zum Beispiel Spikulen vom Typ I oder II, oder auch den Ca II K Fibrilen.

Der zweite Teil der Arbeit widmet sich der Studie der Oszillationen der SCFs mittels einer Wavelet-Analyse. Die erste Analyse konzentriert sich dabei auf die Oszillation der Helligkeit und der Breite der SCFs. An mehreren Positionen entlang der Fibrilen werden diese Oszillationen bestimmt und daraus ihre Perioden und Phasengeschwindigkeiten abgeleitet. Die Oszillationen in beiden Parametern zeigen überlappende Perioden von $32 \pm 17 \mathrm{~s}$ für die Helligkeitsvariation und $36 \pm 25 \mathrm{~s}$ für die Variation der Breite. Die Phasengeschwindigkeit dieser Wellen konnte auf $11_{-11}^{+49} \mathrm{~km} \mathrm{~s}^{-1}$ für die Breite und auf $15_{-15}^{+34} \mathrm{~km} \mathrm{~s}^{-1}$ für die Helligkeit bestimmt werden. Durch die Berechnung des sogenannten cross-power Spektrums zwischen den beiden Oszillationen konnten wir beobachten, dass sie in den meisten Fällen in Phase oder in Gegenphase ablaufen, wobei die gegenphasige Oszillation bei weitem überwiegt. Dies deutet darauf hin, dass beide Oszillationen vom selben Wellentyp verursacht werden. Durch den Vergleich mit der lokalen Schall- und Alféngeschwindigkeit konnten wir schlussfolgern, dass es sich bei den beobachteten Os- 
zillationen um sogenannte fast-sausage-mode Wellen handelt. Enige Fälle sind auch kompatibel mit dem slow-sausage-mode Typ.

Es wurden auch die transversalen Bewegungen der SCFs untersucht. Dabei erhalten wir mittlere Amplituden im Bereich von $2.4 \pm 0.8 \mathrm{~km} \mathrm{~s}^{-1}$ und Perioden von $83 \pm 29 \mathrm{~s}$. Wir konnten die mittlere Phasengeschwindigkeit dieser transversalen Bewegung entlang der SCFs auf $9 \pm 14 \mathrm{~km} \mathrm{~s}^{-1}$ bestimmen. In einigen Fällen pflanzen sich die Wellen in beide Richtungen fort, aber auch stehende Wellen konnten beobachtet werden. Diese Oszillationen können als sogenannte kink-Wellen bezeichnet werden. Mit den berechneten Parametern der Oszillation können diese Wellen einen Energiefluss von $\approx 15 \mathrm{~kW} \mathrm{~m}^{-2}$ beinhalten, eine Energiemenge, die für die Heizung der Chromosphäre ausreichend ist . 


\section{Introduction}

Since the invention of the telescope in the $17^{\text {th }}$ century the solar atmosphere has been subject of observational, theoretical and computational studies that allowed for great advances in understanding of the solar features, and their relation with the solar magnetic field.

Together with the increase of knowledge about the Sun, the techniques and the technologies available to the scientists evolved in time. This led to the discovery of the stratification of the solar atmosphere in the end of the $19^{\text {th }}$ century by using, for example, spectroscopic techniques.

Based on physical properties (temperature, density, spectral emission, ...) the solar atmosphere can be divided in four layers: the photosphere, chromosphere, transition region and the most external layer, the corona (Figure 1.1).

In Figure 1.2 we can see the different physical properties of these layers, the plasma density, temperature, and the electron density as a function of height. Note that this is a vastly simplified representation of the true solar atmosphere and that the values given below are also horizontally averaged (e.g., temperature and density in the corona can vary by over an order of magnitude). The plasma density is characterized by a relatively steady decrease from the photosphere to the top of the chromosphere (from around $10^{-4}$ down to $10^{-9} \mathrm{~kg} \mathrm{~m}^{-3}$ ), and then a drastic decrease in the transition region to values of $10^{-11} \mathrm{~kg} \mathrm{~m}^{-3}$, and an average value of $10^{-12} \mathrm{~kg} \mathrm{~m}^{-3}$ throughout most of the corona. At the same time, the temperature decreases from values near $6000 \mathrm{~K}$ at the visible pho-

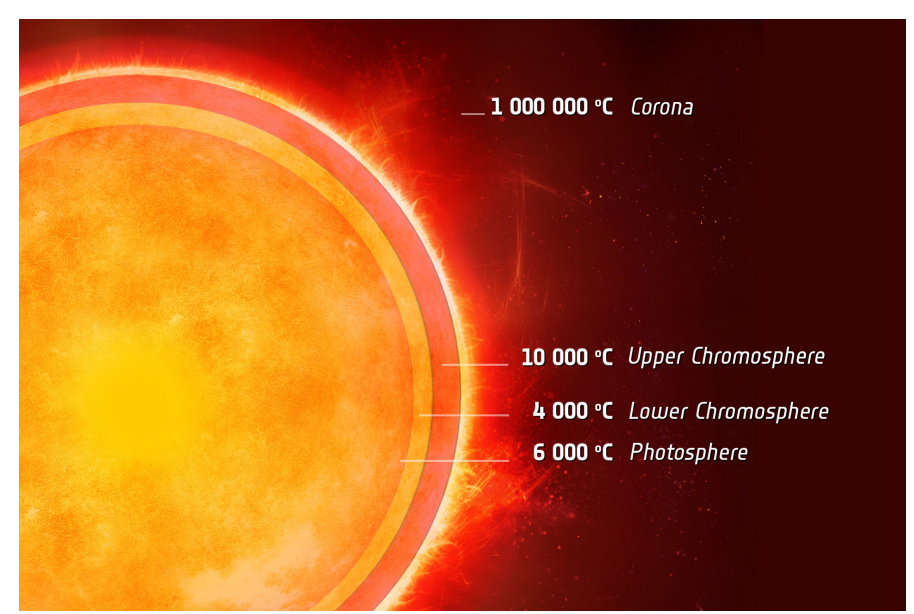

Figure 1.1: Solar atmospheric structure. The different layers are characterized by their characteristic temperatures. Image: ESA 


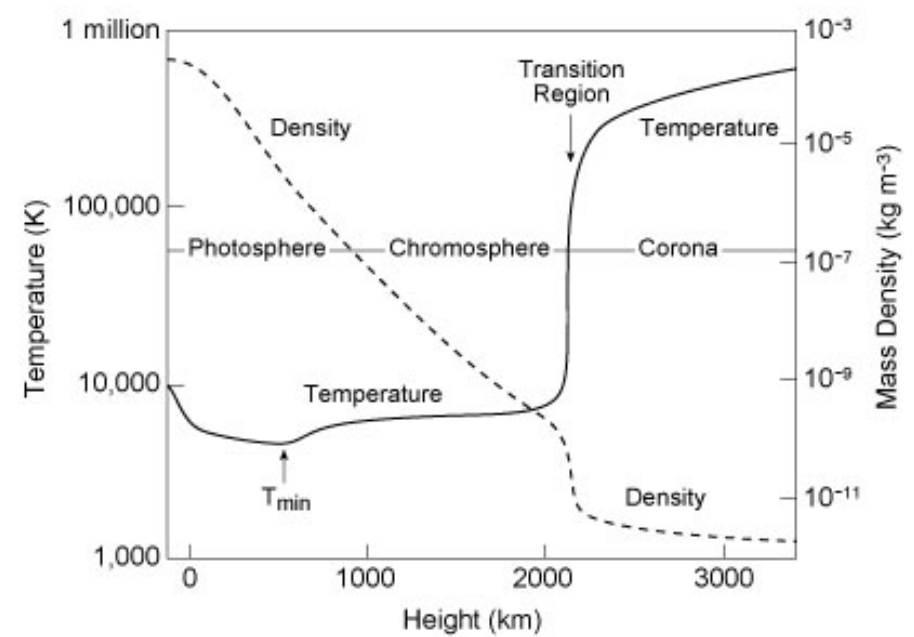

Figure 1.2: Average temperature and density profiles in the solar atmosphere (Courtesy of Eugene Avrett, Smithsonian Astrophysical Observatory).

tosphere to a minimum value of roughly $4400 \mathrm{~K}$ about $500 \mathrm{~km}$ higher up. After that, the temperature increases with height, slowly at first, then extremely rapidly in the narrow, so-called transition region, less than $100 \mathrm{~km}$ thick, between the chromosphere and corona, from about $10000 \mathrm{~K}$ to about one million $\mathrm{K}$. This increase in temperature creates the condition for a strong emission in the $\mathrm{H} \alpha$ line, which in a strict sense is defining the chromosphere. Explaining the causes of this observed increase in the temperature in the outer layers of the solar atmosphere (the chromospheric and coronal heating problem) is still one of the biggest open issues in the solar physics. The electron density decreases by two orders of magnitude in the photosphere, then rises again slightly in the chromosphere, and finally decreases following the same trend as the plasma density. Another important physical property of the solar atmosphere is the plasma $\beta$, defined as the ratio between the gas pressure $(p)$ and the magnetic pressure $\left(\beta=2 \mu_{0} p / B^{2}\right)$. This quantity defines the two different regimes where the dynamics are dominated by the gas $(\beta>1)$ and where they are dominated by the magnetic field $(\beta<1)$. In the Figure 1.3 we see that in the photosphere the plasma $\beta$ is mainly $>1$, whereas it becomes increasingly smaller in the chromosphere and corona.

Due to its location and characteristics the chromosphere plays an important role in the dynamics of the whole solar atmosphere. Large scale structures present in the chromosphere and in the photosphere, like sunspots, $\mathrm{H} \alpha$ filaments, or the network fields have been studied in great detail in the $20^{\text {th }}$ century, but the understanding of these large scale features requires the study of their fine structure. Many of these small-scale structures, in particular fibrils or jet-like structures, play an important role in the coronal dynamics either by the constant plasma flow that they can inject into the upper layers (Pereira et al. 2012), or by the energy that propagates or even dissipates therein Withbroe and Noyes (1977).

The development of the new instruments and new telescopes brought unprecedented spatial, and spectral resolution and temporal stability. A highlight in this development is the SUNRISE observatory (Solanki et al. 2010). SUNRISE takes advantage of the seeingfree observing conditions in the stratosphere, to record data, for example in the $\mathrm{Ca}$ II $\mathrm{H}$ 


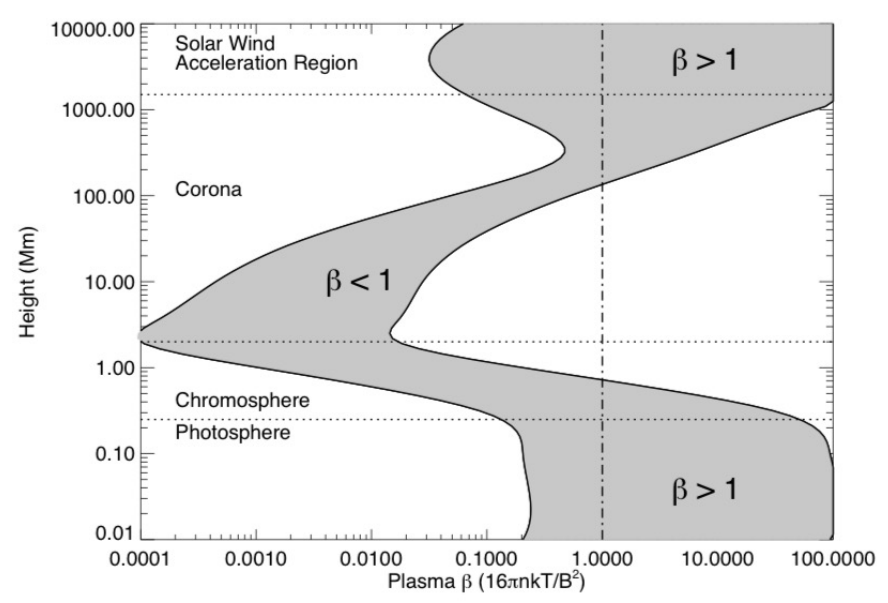

Figure 1.3: Plasma $\beta$ as function of height for magnetic field from 150 to $2500 \mathrm{G}$ represented by the grey area. Taken from Gary (2001).

line, with highest spatial and spectral resolution for a long period of time for the first time.

Within the framework of this thesis, we focus on the analysis of bright elongated structures in the chromosphere that are observed in the data set recorded during the second scientific flight of the SUNRISE observatory. I present their morphological properties and oscillatory behaviour. The outcome of this work will help to improve the understanding of the physical processes in the lower chromosphere and consequently the coupling between the photosphere to the atmospheric layers above.

\subsection{Connection between the photosphere and chromosphere}

The small-scale chromospheric fibrils are the main topic of this thesis. In this chapter we concentrate on the layer, where these structures are rooted, the solar photosphere, and its influence on the chromospheric layers.

The photosphere is the lowest part of the solar atmosphere and many of the most prominent solar features are present there. For example, sunspots, pores or plage regions, that are characterized by the interaction between the photospheric plasma and the magnetic field (Hale 1908) have been observed for centuries. The magnetic field that drives these structures is controlled by the solar dynamo (Brandenburg and Subramanian 2004; Charbonneau 2010). A review summarizing the variety of magnetic features in the solar photosphere is given by Solanki et al. (2006).

Observing the Sun in white light outside active regions shows that the surface is dominated by a granular pattern of bright cells and dark lanes, created by the convective motion of the plasma (see left panel in Figure 1.4). The typical lifetime of these granular cells is around 10 minutes and their sizes are around $1 \mathrm{Mm}$ (Hirzberger 2003). The cells are characterised by central upflows with velocities in the range of $1 \mathrm{~km} \mathrm{~s}^{-1}$. This rising hot plasma cools down at the surface and flows back in narrow intergranular lanes marking the boundaries of the granular cells with typical velocities of $2 \mathrm{~km} \mathrm{~s}^{-1}$. 

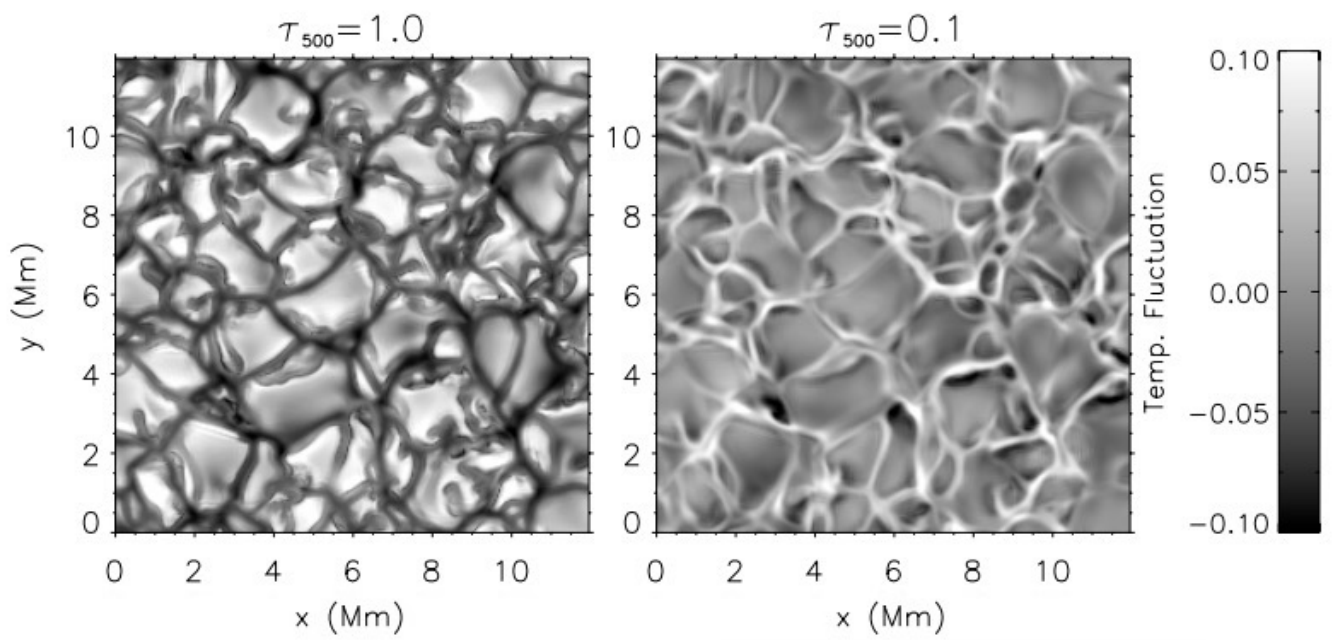

Figure 1.4: The granular pattern of the quiet Sun as seen in temperature fluctuations at constant optical depth. Left: photospheric granulation pattern taken from a magnetohydrodynamical (MHD) simulation at optical depth unity $\left(\tau_{500}=1\right)$; right: reversed granulation pattern from the same simulation at $\tau_{500}=0.1$. Adapted from (Cheung et al. 2007).

The high plasma- $\beta$ causes the convective motions to be essential for the distribution of the magnetic fields close to the solar surface. There in the so-called internetwork part of the quiet Sun, where the so-called small-scale turbulent dynamo creates magnetic fields in the near-surface layers of the Sun (Vögler and Schüssler 2007; Graham et al. 2009). These fields are concentrated in the intergranular lanes by the horizontal motion at the granular surface if the internetwork elements live long enough. The polarities of the internetwork fields in the intergranular lanes are therefore randomly distributed, and opposite polarity intergranular lanes are likely to be connected by small-scale loops, forming a low lying canopy (see Figure 1.5). Since the typical lifetime of granules is in the order of only 10 minutes, these small-scale loops are extremely dynamic. These loops are visible in $\mathrm{H} \alpha$ as dark elongated structures (Mooroogen et al. 2017).

The small scale magnetic flux concentrations that are observed in these quiet-sun regions have lifetimes between the ones of the granules themselves up to around 2 to 4 hours with a typical size of $2 \mathrm{Mm}$ and a magnetic flux up to $10^{18} \mathrm{Mx}$ (Anusha et al. 2017; Smitha et al. 2017).

In the upper layers of the solar photosphere this granulation pattern undergoes a significant change. The dark intergranular lanes become brighter than the granular cells. This pattern is called reversed granulation (see right panel in Figure 1.4), and is well visible in the line core of strong photospheric lines or in chromospheric lines like the $\mathrm{Ca}$ II $\mathrm{H}$ line (Rutten et al. 2004).

In the ascending phase, the overshooting gas expands adiabatically leading to a decrease in temperature. Due to the increase in pressure in the centres of the granules, caused by the accumulation of rising, no longer buoyant gas (the region above the solar surface is convectively stable), the gas is accelerated sideways. At the edge of the granule, where it meets horizontally flowing gas from other granules, pressure builds up again and, the gas starts to falls down into the solar interior being compressed adiabatically. 


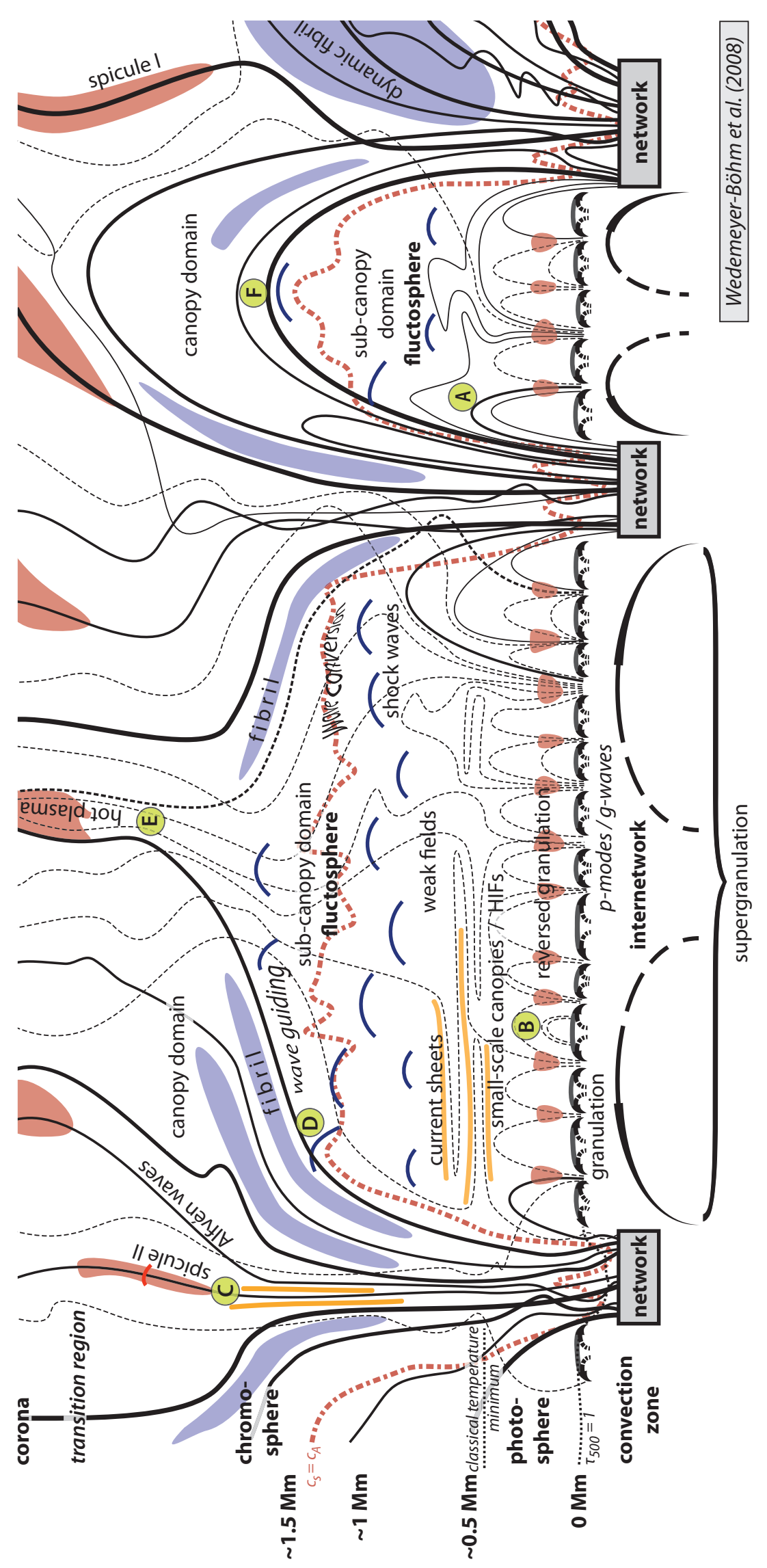

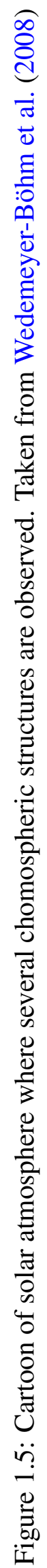


This process of compression increases the gas temperature, creating the reversal of the brightness pattern in layers around $200 \mathrm{~km}$ above the $\tau=1$ level as shown in Figure 1.5 or in $\mathrm{Ca}$ II $\mathrm{H}$ intensity maps.

On a larger scale of around 10 to $50 \mathrm{Mm}$, the magnetic fields in the quiet Sun are organized in the so-called network. The network structure is caused by large convective cells called supergranules (Leighton et al. 1962), having lifetimes in the order of days and typical horizontal motions in the range of $0.5 \mathrm{~km} \mathrm{~s}^{-1}$. The upflow and downflow velocities are in the order of 50 and $100 \mathrm{~m} \mathrm{~s}^{-1}$, respectively. Similarly to what happens in the granulation the horizontal motion concentrates the magnetic field at the edges of the cells, particularly the interception of different cells (Wang et al. 1995; Schrijver et al. 1997). Liu et al. (1994) estimated that the life time of the network elements are about 50 hours. Please note that this value is highly dependant on the used method.

The size range of these magnetic patches varies from 1 to $10 \mathrm{Mm}$ with a typical magnetic flux of about $10^{18}$ to $10^{19} \mathrm{Mx}$ (Brown et al. 2001) and with magnetic fields with strengths in the $1.5 \mathrm{kG}$ range. The network fields might be connected by strong horizontal magnetic field (Lites et al. 2008), the so-called canopy fields (e.g. Jones and Giovanelli 1982; Solanki and Steiner 1990).

To produce the even stronger magnetic field present in sunspots, pores or plage, the emergence of magnetic flux is required. At this moment two main different theories can explain this process. The first one, that for decades was the standard theory, is based on the rising of buoyant flux tubes to the surface (Schüssler et al. 1994). One key element of this model is amplification and conversion of the poloidal magnetic field into a toroidal field in the tachocline by the differential rotation. The high magnetic pressure in these flux tubes causes a low plasma pressure, making the flux tubes buoyant and rising to the surface, where they form bipolar active regions. The field is then dragged by the meridional flow first to the poles and later again to the bottom on the convective zone as a poloidal field and the process repeats. This cycle is the 11 years solar cycle, that is characterised by periods of strong activity at the solar surface alternating with quiet periods (Hathaway et al. 1994).

According to an alternative, distributed dynamo model (see review of Charbonneau 2010) the magnetic field generation occurs all over the convection zone, due to turbulent effects being important in addition to differential rotation. Such a dynamo generates diffuse large-scale fields everywhere in the convection zone. These magnetic fields are postulated to become unstable near the surface and form active regions due to another turbulent effect (Brandenburg et al. 2016).

Active regions containing these strong magnetic fields are observed to live from days up weeks and contain several pores and/or sunspots (see Figure 1.6). They show a wide range in diameter from 15 to $150 \mathrm{Mm}$, and a magnetic flux around $10^{22} \mathrm{Mx}$.

Sunspots and pores are surrounded by so-called plage regions, extending several $\mathrm{Mm}$ around them. They appear as a bright ring within active regions in low-resolution observations. These areas are characterized by a high density of $\mathrm{kG}$ features (Howard and Stenflo 1972; Frazier and Stenflo 1972; Stenflo 1973) in form of small flux tubes (Solanki 1993a) with diameters of around $100 \mathrm{~km}$ or less in the quiet-sun internetwork regions, and several hundred $\mathrm{km}$ in plage regions. Due to the small size of these flux tubes, they can be modelled as a thin flux tube, i.e., where the horizontal size of the tube is smaller than the pressure scale height (Spruit 1976; Defouw 1976). (Buehler et al. 2015) estimate 


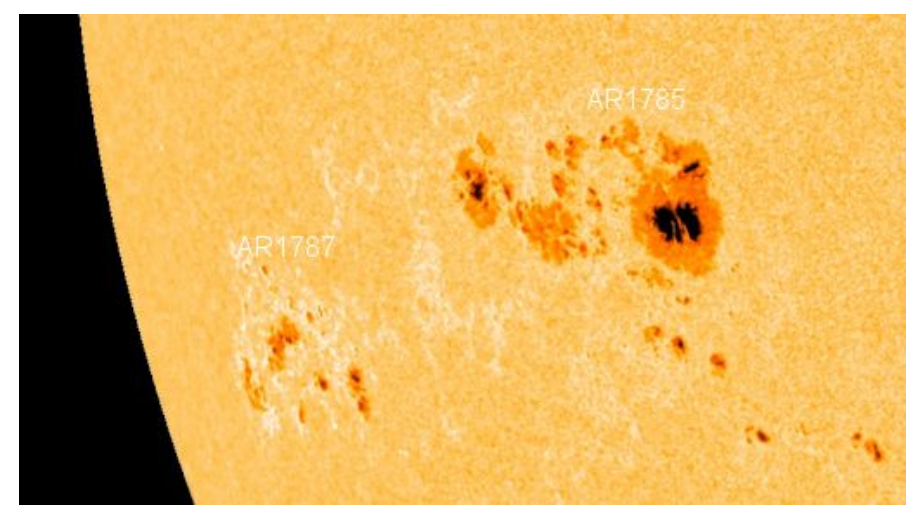

Figure 1.6: Active region constituted by big sunspots and small pores, and surrounded by the bright plage regions. Credit: NASA's Solar Dynamics Observatory.

that the inclinations of the flux tubes are preferentially vertical with angles between 10 to 15 degrees and magnetic fields of $\approx 1500 G$ at $\log (\tau)=-0.9$. This vertical orientation is a result of the buoyancy of the flux tubes (Schüssler 1986).

All these magnetic field concentrations finally reach up to the chromosphere (Pietarila, A. et al. 2010) where they create several different features depending on the characteristics of the magnetic field and their location (Wedemeyer-Böhm et al. 2008). The data analyzed in this thesis are coming from such plage regions.

\subsection{Chromospheric jets and fibril like structures}

As mentioned in Sect. 1, the solar chromosphere is a highly warped layer on top of the solar photosphere with an average thickness of about $1.5 \mathrm{Mm}$ (Wedemeyer-Böhm et al. 2008). In the upper chromosphere the structure and dynamics are dominated by the magnetic field (plasma $\beta<1$ ) contrary to the lower chromosphere and photosphere $(\beta>1)$.

A prominent feature of the solar chromosphere above plage and network fields are small-scale, elongated fibrils, well visible in chromospheric lines like the Ca II H\&K or the $\mathrm{H} \alpha$ lines. In this section we describe these highly dynamic chromospheric structures, appearing in the literature as mottles, fibrils, straws, rapid blue excursions (RBEs), and spicules (see Tsiropoula et al. 2012, and Sect. 1.2.1). These structures possibly play a major rule in the energy and mass transport from the lower to the upper layers of the Sun (see, e.g., Pereira et al. 2012). Observations using filtergraph instruments allow to characterize the morphological properties and dynamics of these structures. More recently, spectroscopic and spectropolarimetric observations allow to infer the physical processes and conditions like density, temperature and plasma flows within these structures.

The SUNRISE data used in this work, reached a new level in terms of spatial resolution. This allows us to apply wave analysis techniques in order to identify the wave modes (that we describe in more detail in section Sect. 1.2.2) present in these elongated structures. According to some models, the energy transported by such waves is high enough to heat the solar chromosphere (Withbroe and Noyes 1977; Vernazza et al. 1981).

Despite all the observational and computational studies, the formation and the role in the mass and energy transport to the solar corona of chromospheric jets and fibril-like 
structures are still not well understood. To improve the understanding it is essential to investigate their detailed morphological properties and their dynamics.

\subsubsection{General properties of small scale chromospheric jet and fibril- like structures}

Figure 1.5 illustrates the complexity of the chromosphere, containing structures of all sizes, from filaments spanning over half a solar radius to the features barely resolvable with modern solar telescopes. In this work I concentrate on the small-scale structures. Here is a short overview of some terms used in the literature:

\subsubsection{Mottles}

Mottles are usually referred as highly dynamic, hair-like jets observed on disk in the quiet Sun in chromospheric spectral lines like $\mathrm{H} \alpha$ or the $\mathrm{Ca}$ II lines. They typically appear as dark, elongated structures, and are best observed in the wings of the $\mathrm{H} \alpha$-line $( \pm 0.5 \AA$ around the line core). However, some observational studies report of the existence of bright mottles in the same location as the dark mottles. One possible explanation suggested by Banos and Macris (1970) is that the bright mottles are the base of the dark structures. Contradicting this explanation, Alissandrakis and Macris (1971) claim that the two types of mottles are completely different structures. With the analysis of the new high resolution observations it has been speculated that the bright mottles are likely to be the bright background under the dark mottles (Tsiropoula et al. 2012).

Mottles are the most common elements seen in $\mathrm{H} \alpha$ that constitute the highly-warped and dynamic chromosphere. They display a complex geometric pattern, outlining the boundaries of the chromospheric network. The latter is well visible in the Ca lines (although the mottles themselves are not). Based on their location and number they can be divided in two different categories. Small groups, called chains, that are created by almost parallel structures emanating along the common boundary of two supergranular cells. Second, larger groups called rosettes, consist of usually radially expanding structures around the common boundary area of three or more supergranular cells. Tanaka (1974) also found that about $30 \%$ of all the dark mottles appear in pairs or can be resolved into double structures in $\mathrm{H} \alpha$ wing observations.

The dimension of mottles spans from 1 to $10 \mathrm{Mm}$ and width around $500 \mathrm{~km}$ (Bray and Loughhead 1974) and they show different shapes: oval, round, filament- or archlike, and lumpy, (Sawyer 1972). The elongated, filament- or arch-like ones are the most common. Due to the highly dynamic behaviour the life time of these structures is difficult to determine. The values range from 3 to 15 min (Bratsolis et al. 1993).

\subsubsection{Fibrils}

Fibrils are small-scale, dark, elongated structures observed on the solar disc in the plage within active regions (see Figure 1.7 for an example). Similarly to mottles they are also highly dynamic. Unfortunately, the same term is also used for two other types of larger scale structures: dark structures (in $\mathrm{H} \alpha$ ) on top of the sunspot penumbrae that expand radially from the centre of the umbra with typical lengths in the range of ten $\mathrm{Mm}$, and struc- 


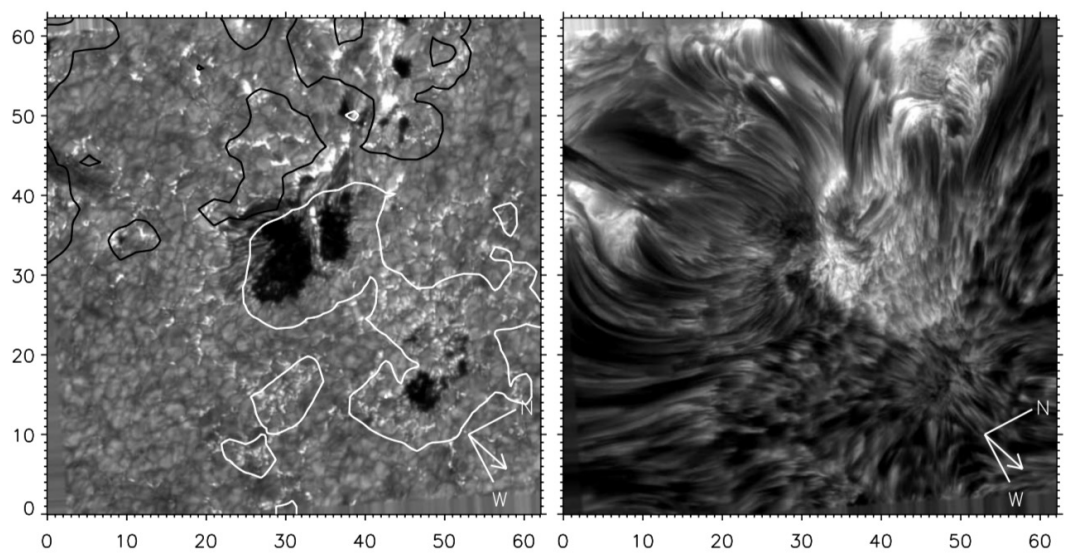

Figure 1.7: Different types of fibrils observed in $\mathrm{H} \alpha$ (right panel). The longer fibrils tend to originate in the umbra / penumbra of the sunspot (see $\mathrm{H} \alpha$ wideband image on the left), the shorter ones are more concentrated in the plage of the active region. Adapted from De Pontieu et al. (2007).

tures that do not show a jet-like behavior in the vicinity of active region plage (Tsiropoula et al. 2012). The latter two are probably part of a low-lying canopy connecting the opposite polarity areas within an active region. This thesis analyses in detail the short fibrils in the plage of active regions.

The analysis of filtergrams of $\mathrm{H} \alpha$ fibrils in plage regions shows their structural similarity to the ones in the quiet Sun. For example, structures similar to rosettes and mottles in the quiet Sun, rooted in network bright points (NBPs), are also existing in plage regions at smaller scales (Tsiropoula et al. 2012). The principal differences between the two regimes are on the one hand a larger number density of small-scale magnetic field concentrations in plage compared to the number density of NBPs and on the other hand the bigger size of the fibrils observed in plage than in the network.

This possible relationship between the structures observed in the quiet and active chromosphere was analyzed by Foukal (1971b). He proposed that there is a gradual change between the quiet-sun mottles, oriented close to the vertical, and the more horizontally oriented fibrils in plage regions.

Fibrils are not only visible in the $\mathrm{H} \alpha$ line observed in active regions, but also in other chromospheric lines like Ca II $\mathrm{K}$ as bright structures. A nice example is the publication of Pietarila et al. (2009) who used high resolution images obtained at the SST with a narrow band filter $(1.5 \AA)$ centered at the Ca II K line. They identify that the endpoints of some of these very thin structures coincide with magnetic field concentrations in the photosphere. This connection is more difficult to establish in strong plage regions due to the high density of magnetic field concentrations. In such regions, like the one in box B of Figure 1.8, where we expect the magnetic field to be more vertical, the identified fibrils are smaller and denser than in the rest of the field of view of Figure 1.8.

At the boundaries of the plage regions the fibrils are then oriented radially outwards around the active region towards the quiet Sun. There they are longer and more inclined. Pietarila et al. (2009) concluded that these bright structures are formed by the underlying magnetic field - a result that is also suggested by Jafarzadeh et al. (2017) for the bright fibrils observed in $\mathrm{Ca}$ II H. They found a strong correlation between the fibril orientation 


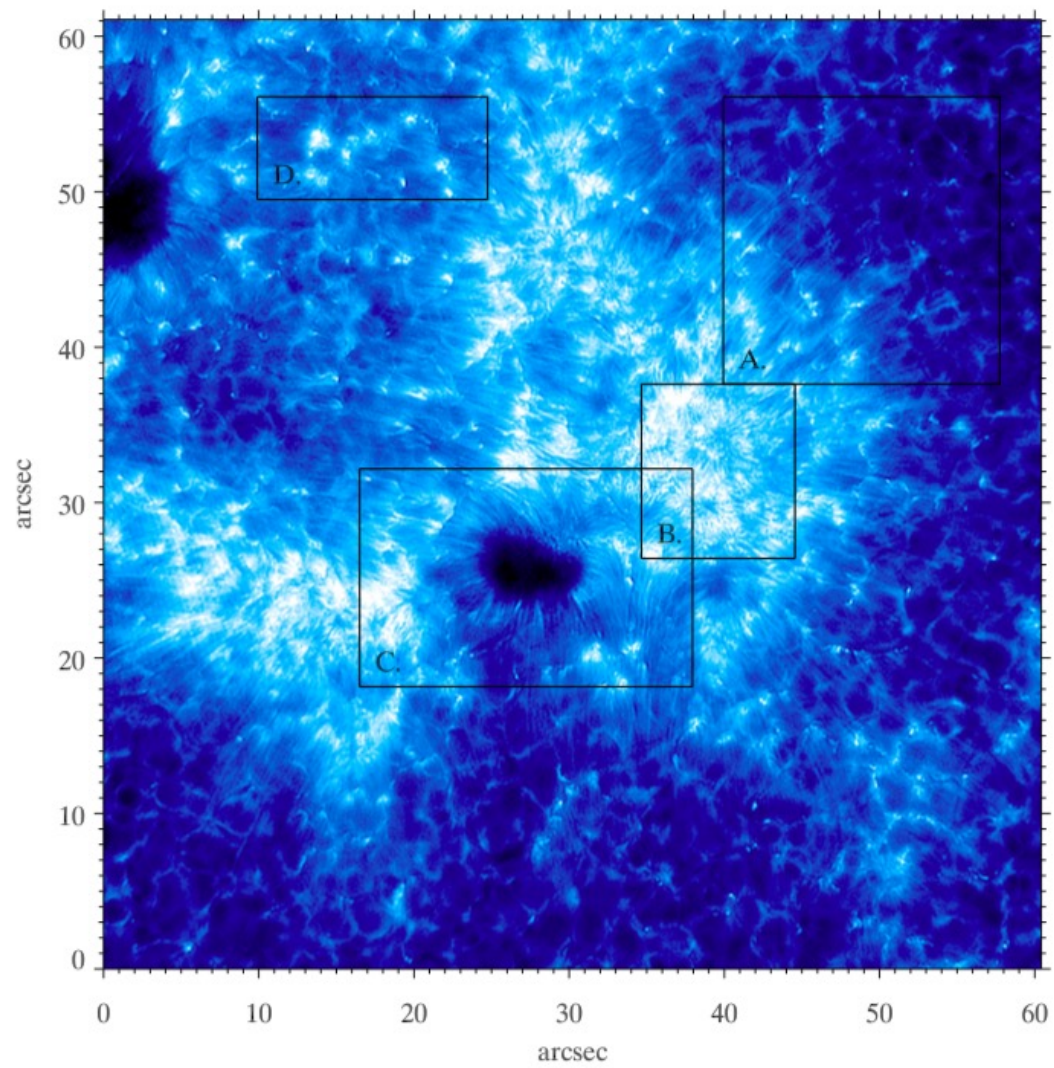

Figure 1.8: Ca II K filtergram of a small active regions. The boxes identify different solar regions of interest. Box $\mathrm{A}$ is placed over the boundary between the plage region and the quiet Sun. Box B shows only the plage region. Box C contains a small pore. Box D represent a region between two magnetic polarities.

Taken from Pietarila et al. (2009).

and the magnetic field lines obtained by magnetic field extrapolation from photospheric vector magnetograms.

Foukal (1971a) for the first time compared some morphological properties of $\mathrm{H} \alpha$ fibrils and spicules. He saw a similarity of these two structures in terms of lifetime, density, temperature, and velocity, but reported on higher lengths for spicules and stronger magnetic fields in fibrils. However, this description is still speculative.

The typical fibrils show a huge range of their morphological properties. The lifetime varies from less than a minute to 20 minutes, the width between few hundreds of $\mathrm{km}$ to $2 \mathrm{Mm}$, and the length between few hundreds of $\mathrm{km}$ to $15 \mathrm{Mm}$. A subcategory of these fibrils are the so-called dynamic fibrils, (DF, Grossmann-Doerth and Schmidt 1992; Hansteen et al. 2006). These DFs are shorter than the typical fibrils and shorter lived. They are mainly observed in the vicinity of plage regions or strong magnetic field concentrations.

DFs, when observed in $\mathrm{H} \alpha$, have life times of 120 to $650 \mathrm{~s}$ with an average value of $290 \mathrm{~s}$, typical sizes of 400 to $5200 \mathrm{~km}$ and a mean length of $1250 \mathrm{~km}$, and widths between 120 and $380 \mathrm{~km}$ that remain almost constant during the fibril lifetime. De Pontieu et al. (2007) identify a variation of these properties depending on their location, conclud- 
ing that the fibrils observed in denser plage regions are shorter in length and lifetime. Pietarila et al. (2009) estimated also some properties for the bright fibrils observed in the Ca II K line. They estimate the width to lie between 75 and $150 \mathrm{~km}$ and a length of approximately $900 \mathrm{~km}$. Anan et al. (2010) used observations in Ca II H (band width of $3 \AA$ ) from the Hinode Solar Optical Telescope (SOT) / Broadband Filter Imager (BFI) (Tsuneta et al. 2008) to analyze the brightness and the morphological properties of these structures. They identify two classes of brightness variations, where $80 \%$ of them show a parabolic evolution, i.e. a slow brightening followed by a slow fading, and $10 \%$ a "fade out" behavior. The lengths of these fibrils were estimated to be between $0.8 \mathrm{Mm}$ and $2.3 \mathrm{Mm}$, and the lifetimes between $100 \mathrm{~s}$ and $400 \mathrm{~s}$.

\subsubsection{Rapid Blueshifted Excursions}

Rapid Blueshifted Excursions (RBEs) are observed on disk structures that are characterized by a blueshift or a fast, asymmetric broadening in the blue wing of chromospheric lines like $\mathrm{H} \alpha$ and Ca II IR (Langangen et al. 2008). Common properties observed in both spectral lines are their short mean lifetime of $45 \pm 13 \mathrm{~s}$ and a typical width of $0.5 \mathrm{Mm}$. However, some of the properties depend on the line the RBEs are observed in: the ones observed in $\mathrm{H} \alpha$ are on average longer than the ones observed in Ca II IR (3 Mm compared to $2 \mathrm{Mm})$. The blueshift is approximately $35 \mathrm{~km} \mathrm{~s}^{-1}$ in $\mathrm{H} \alpha\left(15 \mathrm{~km} \mathrm{~s}^{-1} \mathrm{Ca}\right.$ II $)$, and the Doppler width of the blueshifted component is $13 \mathrm{~km} \mathrm{~s}^{-1}$ compared to $7 \mathrm{~km} \mathrm{~s}^{-1}$ in Ca II (Langangen et al. 2008; Rouppe van der Voort et al. 2009).

One possible explanation for this rapid blueshifts was suggested by Langangen et al. (2008) who used a Monte Carlo simulation to show that the observed lineshifts are likely to be caused by a combination of different orientations of spicules and changes in opacity in the upper chromosphere.

Using data from the CRisp Imaging SpectroPolarimeter (CRISP; Scharmer et al. 2008) at the Swedish 1-m Solar Telescope (SST Scharmer et al. 2003a), (Wang et al. 1998) analyzed in detail very similar, but longer lived events in $\mathrm{H} \alpha$ and Ca II IR. They estimate that the density of these structures if observed at the limb is around 1.9 per linear

arcsecond. Judge et al. (2011) estimate that the number of RBEs in the Sun is $\approx 10^{5}$ at any given time. They also suggested that the RBEs could be the counterparts of type-II spicules on disk, since they are visible in both spectral lines.

\subsubsection{Spicules}

Spicules are bright, elongated, jet-like structures that are observed off-limb (Beckers 1968, 1972; Sterling 2000) in lines like $\mathrm{H} \alpha$ and $\mathrm{Ca}$ II H, showing the same behavior in both lines (Pereira et al. 2016). The first to use the term spicule was Roberts (1945) when analyzing $\mathrm{H} \alpha$ off limb chronograph images. He observed that these jets reach heights of around $10 \mathrm{Mm}$ and last between 2 and 11 minutes. Even earlier, in 1877, observations of jet-like structures were already reported by Father Angelo Secchi. Lippincott (1957) classified spicules in two categories. The first one, called porcupine, contains spicules with a size of $18 \mathrm{Mm}$. The porcupines tend to be oriented radially outwards from a common point. The second category, called wheat, has a size of $140 \mathrm{Mm}$. The wheat have a dominant inclination direction with respect to the limb direction. She also computed 
for the first time the propagation velocity of the material within these spicules that range from a few $\mathrm{km} \mathrm{s}^{-1}$ up to $60 \mathrm{~km} \mathrm{~s}^{-1}$.

After these early discoveries, spicules were for decades not in the focus of scientific interest. One reason for this might be that the lack of high-resolution data made it difficult to gain new insight into these jet-like structures, for example to specify the relation of the off-limb spicules to their on-disk counterparts. These counterparts must exist, but are extremely difficult to be observed, posing a challenge even for modern solar observations. Some early studies reported that spicules are present only in quiet-sun regions at the limb. However, jet-like structures have also been found in the quiet Sun on disk. These structures are called mottles (see Sect. 1.2.1.1), and they possibly present the on-disk counterparts of spicules. Other possible candidates are for example dynamic fibrils (see Sect. 1.2.1.2). All of these structures are important contributors to the chromospheric activity.

Using high-resolution data taken with the Hinode BFI of the SOT (Tsuneta et al. 2008) in the Ca II H line, De Pontieu et al. (2007) categorized spicules into two classes with different physical properties. They call these two classes type-I and type-II spicules. Note that this classification is different from the porcupine and wheat classification of (Lippincott 1957).

Type-I spicules are characterized by a parabolic brightness evolution in the sense that if a slit is put along the axis of the spicule and its evolution is plotted then it shows a parabolic track vs. time, i.e., it decelerates while rising to a maximum height and then accelerates while falling. This is reminiscent of a ballistic trajectory of emitting material along the spicule and a similar behavior during the rise and fall phase. They are relatively long-lived with a lifetime from 150 to $400 \mathrm{~s}$ and propagation velocities from 15 to $40 \mathrm{~km} \mathrm{~s}^{-1}$. They reach maximum heights from 2.5 to $11 \mathrm{Mm}$, and inclination angles between vertical to the solar surface and up to 40 degrees. This type of spicules is commonly observed in active regions (Pereira et al. 2012). Their properties indicate that a magnetoacoustic shock wave can be a possible driver of these structures. Also some studies suggest that mottles are the possible on-disk counterparts of these structures (Rouppe van der Voort et al. 2007).

Type-II spicules just show a brightening during the rising phase, fading away after reaching their maximum length. They are shorter lived, with lifetimes ranging only from 20 to $150 \mathrm{~s}$, and have higher propagation velocities from 30 to $110 \mathrm{~km} \mathrm{~s}^{-1}$. Their maximum heights range from 1.5 to $12 \mathrm{Mm}$, and inclination angles are similar to the ones of type-I spicules. Usually, they are observed in quiet-sun regions or coronal holes showing some small differences between both locations in terms of lifetime and velocities (Pereira et al. 2012). Comparing some morphological properties of RBEs (see Sect. 1.2.1.3) and type-II spicules (see Table 1.1) a similarity between these structures is evident. This indicates that RBEs can be the on-disk counterparts of this type of spicules.

Despite the spicule's ubiquitous presence in all solar lower atmospheric observations the estimation of their number density is still a question under debate. Due to the fact that the number of observed spicules is highly dependent on the quality of the observation, the estimations still disagree by around one order of magnitude. (Beckers 1972) estimated that the number of spicules always present on the whole solar surface is around $10^{6}$ spicules at the lowest layers with a decreasing number with increasing height. Athay (1976) computed their number at a height of around $5 \mathrm{Mm}$ at any given time to be 56000 . 
More recent studies analyze in more detail the distribution of type I and II spicules. Judge and Carlsson (2010) estimate that at a given moment there exist $2 \times 10^{7}$ type-II spicules, distributed to 1600 spicules within each supergranule. On the other hand Moore et al. (2011) estimate that this number is much smaller (only around 50 spicules per supergranule). This discrepancy shows that the detection of these structures still poses a challenge even for modern solar observations, possibly requiring improved instrumentation to settle this issue in the future.

Table 1.1: Overview of small scale elongated chromospheric structures

\begin{tabular}{|c|c|c|c|}
\hline property & mottles & \multicolumn{2}{|c|}{ fibrils } \\
\hline length & 1 to $10 \mathrm{Mm}$ & \multicolumn{2}{|c|}{ few hundreds of $\mathrm{km}$ to $15 \mathrm{Mm}$} \\
\hline width & $500 \mathrm{~km}$ & \multicolumn{2}{|c|}{ few hundreds of $\mathrm{km}$ to $2 \mathrm{Mm}$} \\
\hline lifetime & 3 to $15 \mathrm{~min}$. & \multirow{2}{*}{\multicolumn{2}{|c|}{$\begin{array}{l}\text { less than a min. to } 20 \mathrm{~min} \text {. } \\
\text { on disk }\end{array}$}} \\
\hline location & on disk & & \\
\hline spectral lines & $\mathrm{H} \alpha, \mathrm{Ca}$ II & \multirow{2}{*}{\multicolumn{2}{|c|}{$\begin{array}{c}\mathrm{H} \alpha \\
\text { active regions }\end{array}$}} \\
\hline photospheric root & edges of supergranules & & \\
\hline & $\mathrm{RBE}$ & spicule I & spicule II \\
\hline length & $1.2 \mathrm{Mm}$ & $0.5-3 \mathrm{Mm}$ & $1-6 \mathrm{Mm}$ \\
\hline width & $500 \mathrm{~km}$ & $100 \mathrm{~km}$ & $100 \mathrm{~km}$ \\
\hline lifetime & $45 \pm 13 \mathrm{~s}$ & $100-500 \mathrm{~s}$ & $20-100 s$ \\
\hline location & on disk & $\operatorname{limb}$ & $\operatorname{limb}$ \\
\hline spectral lines & $\mathrm{Ca}$ II IR and $\mathrm{H} \alpha$ & $\mathrm{H} \alpha, \mathrm{Ca}$ II H & $\mathrm{H} \alpha, \mathrm{Ca}$ II H \\
\hline photospheric root & Edges of network & & \\
\hline
\end{tabular}

\subsubsection{MHD waves}

Common to most of the structures described in the previous section is that they can show oscillatory behaviours in the form of transversal, longitudinal or torsional magnotohydrodynamical (MHD) waves. In this section I give a brief overview of the MHD wave types relevant for the small-scale elongated structures, their properties and their relevance to important questions like their ability to act as a transport agent for energy to heat up the higher layers of the solar atmosphere.

Different types of oscillations have been observed in the solar atmosphere in magnetised and field-free regions (see, e.g., Narayanan 2013). The restoring forces are the magnetic field (Bogdan et al. 2003), the gravity, and pressure.

In this chapter we concentrate on the description of waves with the magnetic field as the restoring force, since the elongated, small-scale structures are clearly of magnetic nature (Jafarzadeh et al. 2017). Depending on the type of the excitation, the interplay between the pressure and the magnetic properties we can identify 3 different types of oscillations or modes: kink-, sausage- and Alfvén-type waves that are illustrated in Figure 1.9.

In the ideal case of a static vertical thin flux tube embedded in a stratified and isothermal medium, implying a constant pressure scale height $\left(H_{p}\right)$ inside and outside of the tube, the so-called thin flux-tube approximation is valid. The height dependence $(z)$ of 

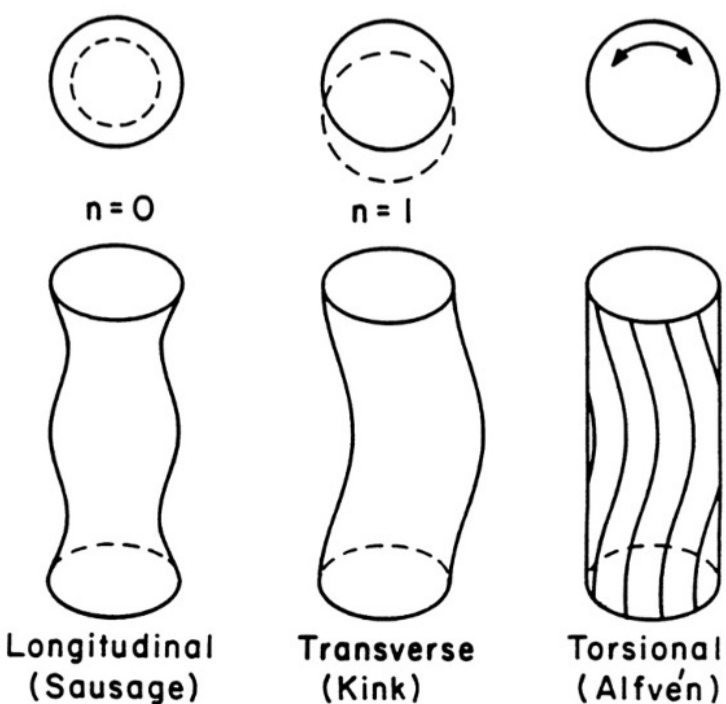

$n=1$

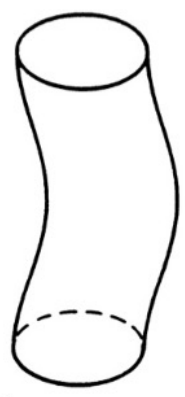

Transverse

(Kink)

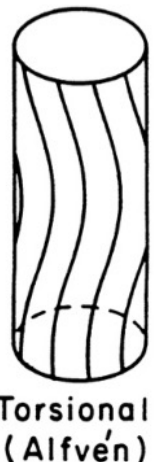

Figure 1.9: Cartoon of the possible MHD wave modes in a thin flux tube from Solanki (1993b).

the tube pressure $(p)$, magnetic field $(B)$, and radius $(R)$ is given by (see Solanki 1993b; Defouw 1976)

$$
\begin{gathered}
p(z)=p e^{-z / H_{p}} \\
B(z)=B_{0} e^{-z / 2 H_{p}} \\
R(z)=R_{0} e^{-z / 4 H_{p}}
\end{gathered}
$$

The equations 1.1 and 1.2 show that the pressure and magnetic field strength decrease exponentially with increasing height, whereas equation 1.3 shows that the radius of the flux tube increases with height.

The oscillations of and within such a flux tube can be studied assuming a small adiabatic perturbation with a velocity that is small compared to the propagation speed of the perturbation. The wave velocity can be described by

$$
v(z, t)=v_{0} e^{z / 4 H_{p}} e^{\mathrm{i}(w t-k z)}
$$

where $w$ is the frequency and $k$ the wave number. This equation is valid for all 3 wave modes and allows us to derive the respective dispersion relations.

The Alfvén mode shows a linear dispersion relation:

$$
w=v_{A} k
$$

where $v_{A}$ is the Alfvén speed that is defined as

$$
v_{A}=\frac{B}{\sqrt{4 \pi \rho}}
$$


The Alfvén mode is characterized by an incompressible torsional motion and can be excited by flux tubes twisted by rotating flows.

For the kink mode the dispersion relation is

$$
w^{2}=C_{K}^{2} k^{2}+w_{c, k}^{2}
$$

where $C_{k}$ is the kink mode propagation velocity for high frequencies with respect to the kink cutoff frequency, and $w_{c, k}^{2}$ is the kink cutoff frequency. This type of mode is characterised by a transversal displacement of the flux tube and is excited for example by the buffeting of granules.

The dispersion relation for the sausage mode is given by

$$
w^{2}=C_{T}^{2} k^{2}+w_{c, T}^{2}
$$

where $C_{T}$ is the so-called tube speed for high frequencies compared to the cutoff frequency $\left(w_{c, T}^{2}\right)$. This mode is characterized by longitudinal motions along the tube and can be excited by tube compression from opposite sides.

Some of these modes can be observed in the structures described in Sect. 1.2.1 and are summarized in Table 1.1.

The relevance of these waves to small-scale fibrilar structures is described in three examples described in the following subsections. For a more complete review please see Zaqarashvili and Erdélyi (2009); Rutten (2012); Tsiropoula et al. (2012).

\subsubsection{Kink waves in dynamic fibrils}

The first observation of kink waves in the solar atmosphere was made by Pasachoff et al. (1968), who found them in $\mathrm{H} \alpha$ fibrils. Since then this type of oscillation has been observed in several structures. They are of high relevance for solar physics research, since recent studies suggest that kink waves, at least when observed in the photosphere or chromosphere, have enough energy to heat the quiet corona (He et al. 2009; Jafarzadeh et al. 2017)

Pietarila et al. (2011) analyzed dynamic fibrils observed in the chromospheric Ca II $8542 \AA$ line using CRISP. They were able to analyze the transverse motions of these structures in time, and clearly identified these motions to be kink waves. The waves have periods between 2 and 3 minutes with a mean value of $135 \mathrm{~s}$, an apparent speed of $1 \mathrm{~km} \mathrm{~s}^{-1}$, and a phase speed of around $290 \mathrm{~km} \mathrm{~s}^{-1}$. Using the approximation of a vertical thin flux tube and the expression given by Nakariakov and Verwichte (2005) that relates the magnetic field and the wave period they were able to estimate the magnetic field strength inside the fibrils to vary from 220 to $330 \mathrm{G}$ from one fibril to another. All these values are similar to the ones observed by He et al. (2009) for spicules at heights below $2 \mathrm{Mm}$.

\subsubsection{Alfvén waves in spicules}

By analyzing off-limb Ca II H images taken by Hinode/SOT, de Pontieu et al. (2007) observed that spicules show an ubiquitous transverse oscillatory behavior. Comparing the observation with the results of a self-consistent 3D radiative MHD simulation, they conclude that this displacement is caused by Alfvén waves propagating along the spicules 
that are excited in the photosphere by the granular motion. The computed periods for these waves were between 100 and $500 \mathrm{~s}$ with amplitudes that range from 10 to $25 \mathrm{~km}$. In the same study they estimate that these waves can transport enough energy flux to the solar corona and drive the solar wind and possible contribute to the quiet corona heating.

\subsubsection{Sausage waves in $\mathrm{H} \alpha$ fibrils}

Morton et al. (2012) used $\mathrm{H} \alpha$ data taken by the Rapid Oscillations in Solar Atmosphere (ROSA) imager Jess et al. (2010) to analyze intensity and width variations along the $\mathrm{H} \alpha$ fibrils. They found an anti-phase periodic behaviour of these two quantities with periods of $197 \pm 8 \mathrm{~s}$, phase speeds of $67 \pm 15 \mathrm{~km} \mathrm{~s}^{-1}$ and apparent velocity amplitudes of 1-2 $\mathrm{km} \mathrm{s}^{-1}$. The observed perturbation is traveling at speeds similar to the Alfvén speed that leads to the interpretation of these waves as a fast MHD sausage mode. The antiphase correlation between the variation in intensity and width illustrates the compressive nature of this wave mode.

The authors also conclude that the sausage wave is in the so-called leaky regime. That means that the wave can radiate energy away from the wave guide, i.e., the magnetic flux tube, with decay times on the order of the wave period. It is the task of further studies to perform a careful analysis of the amount of energy that is carried and dissipated by this type of wave.

\subsection{The SunRISE Observatory}

The SUNRISE observatory (Solanki et al. 2010; Barthol et al. 2011) is a balloon-borne scientific mission with a 1-meter Gregorian telescope (see Figure 1.10). Its scientific goal is the observation of the photosphere and the chromosphere at highest spatial resolution and with long temporal stability. The main science goals of Sunrise as defined in Barthol et al. (2011) are:

1. What are the origin and the properties of the intermittent magnetic structure, including the kilo-Gauss concentrations?

2. How is the magnetic flux brought to and removed from the solar surface? What is the role played by local dynamo action and reconnection processes?

3. How does the magnetic field assimilate and provide energy to heat the upper solar atmosphere?

4. How does the variable magnetic field modify the solar brightness?

Questions (1) and (3) are directly related to the topic of this thesis. The small scale structures described in this work outline the connection between the photosphere and the chromosphere and are of high relevance for answering the questions related to the energy transport to the higher layers of the solar atmosphere. A key will be to investigate how these structures interact and how they influence the energy balance between the magnetic field, plasma motion, heat and radiation transfer.

The location of the observatory in the stratosphere allows for observations in the ultraviolet wavelength region from 200 to $400 \mathrm{~nm}$, a region not accessible from the ground. In 
addition, the earth atmosphere at this height provides almost seeing-free conditions which allowed for the recording of the highest quality data in this spectral range ever.

The observatory flew twice. The first flight lasted from 8-13 June 2009. SUNRISE was launched from Kiruna, northern Sweden, and landed on Somerset island, northern Canada and with a mean altitude of $36 \mathrm{~km}$ (Solanki et al. 2010). The second flight took place from 12-17 June 2013. SUNRISE was launched from Kiruna, northern Sweden, and landed on the peninsula of Boothia in northern Canada, with mean flight altitude of 36 $\mathrm{km}$ (Solanki et al. 2017). The technical differences between the first and the second flight where small. Mainly some of the instrumentation were updated and new UV filters were used.

The scientific payload of SUNRISE consists of two instruments: the SUNRISE Filter Imager (SUFI Gandorfer et al. 2011) for observations in the range between 200 and $400 \mathrm{~nm}$, including the Mg II K and Ca II H lines broad with FHWM of $1.8 \AA$ and narrow band with FHWM of 1.1 A filter) lines.

The second instrument is the Imaging Magnetograph eXperiment (IMaX Martínez Pillet et al. 2011), based on a tunable Fabry-Pérot filter sampling various wavelengths in the highly Zeeman-sensitive Fe I line at $525.02 \mathrm{~nm}$.

The work described in this thesis focuses on the analysis of SUFI filtergrams. In this section we briefly describe the instrument SUFI (see Sect. 1.3), and the reduction of the data gathered by this instrument (Sect. 1.3.2), with special focus on the observations in the $\mathrm{Ca}$ II $\mathrm{H}$ line.

The two SUNRISE flights resulted in many high-impact scientific articles, and in two special issues of the Astrophysical Journal Supplements (ApJS). Here I repeat the summary of the articles of the second special issue as given by Solanki et al. (2017). This literal repetition also contains the summary of the two papers being part of this thesis (see Sect. 2 and Sect. 3):

1. Centeno et al. (2017) analyzed emerging flux regions with a detailed description of the interrelated dynamics of the gas and the field, including the

reports on two emerging flux events, describing in greater detail than previously possible the interrelated dynamics of the gas and the field, as well as the likely occurrence of magnetic reconnection during the emergence.

2. The properties of a likely siphon flow and of the small, initially low-lying loop connecting magnetic elements with a pore are deduced by Requerey et al. (2017a) and the 3D structure of the magnetic field lines and hence of the flow vector are determined.

3. The properties and dynamics of moving magnetic features (MMFs) on one side of the largest pore in the IMaX FOV are deduced by Kaithakkal et al. (2017) and contrasted with the properties of MMFs around sunspots.

4. The proper motion of magnetic bright points in different parts of the quiet Sun and of an active region are analyzed by Jafarzadeh et al. (2017). They find very different behaviors depending on the location, with the features moving strongly superdiffusively in the internetwork, diffusively in the network and in between these extremes in the active region. 
5. Riethmüller et al. (2017) present a novel inversion technique employing magnetohydrodynamic (MHD) simulations to provide the model atmospheres needed to compute synthetic Stokes vectors that reproduce the observed Stokes parameters. They illustrate the quality of the inversions by applying the technique to the SUNRISE II polarimetric data.

6. The properties of the slender fibrils dominating the SUFI Ca II H images are determined by Gafeira et al. (2017b), who show that the fibrils live much longer than a simple analysis would suggest, if one takes into account that they often fade away and reappear after some time.

7. The discovery of ubiquitous transverse waves travelling along these fibrils and carrying copious amounts of energy is reported by Jafarzadeh et al. (2017), while Gafeira et al. (2017a) present the discovery of compressible waves travelling along the fibrils, which they identify as sausage waves.

8. Jafarzadeh et al. (2017) find evidence that these slender fibrils seen in Ca II H outline a canopy of magnetic field lying below that known from $\mathrm{H} \alpha$ and Ca II 8542 A fibril observations.

9. Chitta et al. (2017) observed that coronal loops are rooted in regions with mixedpolarity fields. They provide evidence for flux cancellation and presence of inverse Y-shaped jets (signatures of magnetic reconnection) at the base of coronal loops that might supply (hot) plasma to the overlying coronal loop. They suggest a revision of the traditional picture in which each loop footpoint is smoothly connected to unipolar regions on the solar surface.

10. Danilovic et al. (2017) compare an Ellerman bomb observed by SUNRISE II with a similar, simulated event in which magnetic reconnection occurs at the location of emerging flux. The 3D radiation-MHD simulation reveals the complexity of the underlying physical process and the limitations of the observational data. Thus, the SUNRISE/IMaX data cannot determine the height at which magnetic reconnection takes place. The authors also show, however, that the velocity and magnetic vector measured at the high resolution of SUNRISE/IMaX reveals how shortcomings of the MHD simulations can be overcome.

11. Wiegelmann et al. (2017) have computed general linear magneto-static equilibria of the magnetic field and gas using the SUNRISE II/IMaX observations as a boundary condition. In this way they obtain the magnetic field structure in the upper atmosphere without having to assume the validity of the force-free assumption in the solar photosphere. They computed linear magneto-static equilibria for all the IMaX frames of the active region, without the problems faced when modeling the magnetic field in different atmospheric layers of the quiet Sun.

12. Such papers include the investigation by Requerey et al. (2017b) in which the authors uncover the tight connection between concentrated magnetic fields and convectively driven sinks in the quiet Sun. 
13. Kahil et al. (2017) probe the relationship between brightness contrast at UV and visible wavelengths and the magnetic flux in the quiet Sun, finding that the contrast keeps increasing with magnetic flux, unlike most earlier observational results, but in qualitative agreement with MHD simulations.

14. Jafarzadeh et al. (2017) characterize the wave modes observed at two heights in magnetic bright points, including both compressible waves seen in brightness fluctuations and transverse waves obtained from proper motions.

15. The short travel times suggest large wave speeds. A new estimate of the flux emergence rate in the quiet Sun is obtained by Smitha et al. (2017). Compared with the emergence rate deduced from Hinode/SOT data using the same technique, the emergence rate obtained from SUNRISE I data is around an order of magnitude larger.

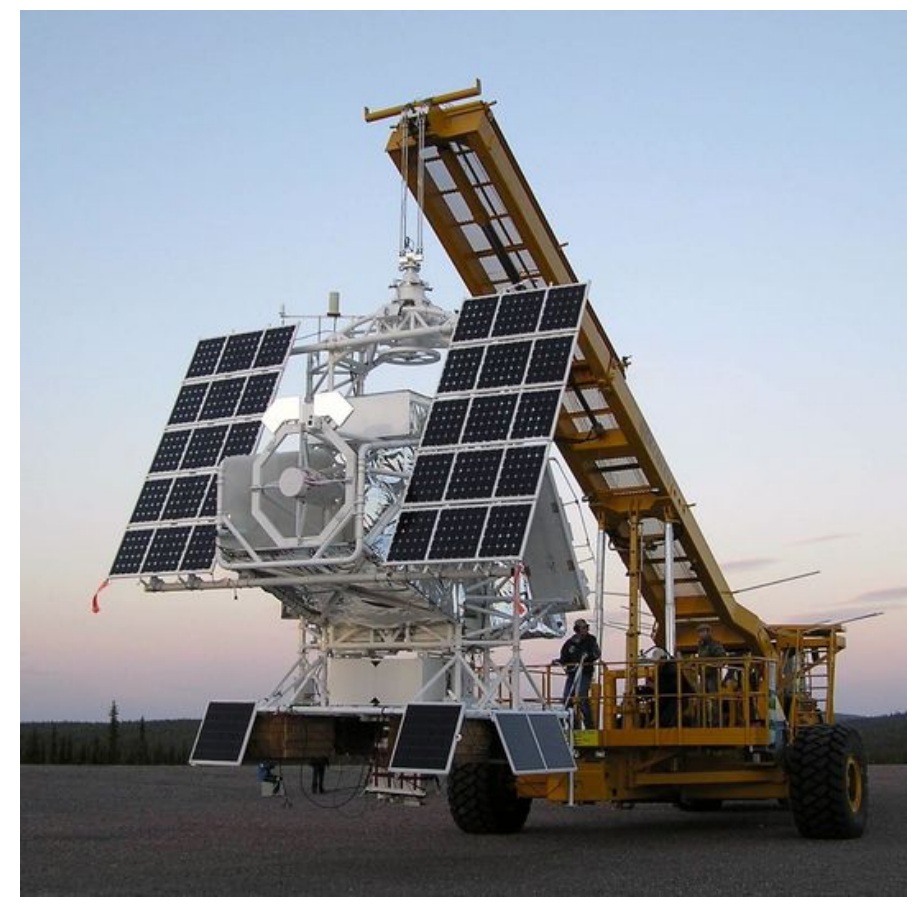

Figure 1.10: SUNRISE observatory on the launch pad in Kiruna before the first launch in June 2009. From Barthol et al. (2011)

\subsubsection{SUNRISE Filter Imager (SUFI)}

To fulfill the SUNRISE scientific goals described in the previous section, SUFI was designed to observe the solar photosphere and chromosphere at the diffraction limit of the 1-meter telescope, which corresponds to a resolution of $0.05 \operatorname{arcsec}(35 \mathrm{~km}$ on the Sun) at $214 \mathrm{~nm}$. With this requirement SUFI was designed to take images providing information about small-scale structures, and the kinetic and thermodynamic properties of the plasma within these structures. 
SUFI can take images at 3 different spectral bands using five filters. During the second SUNRISE flight these filters were: (1) around $214 \mathrm{~nm}$ with a full-width at half maximum (FWHM) of $10 \mathrm{~nm}$ probing the upper photosphere and lower chromosphere, (2) a filter around the $\mathrm{Mg}$ II K line at $279.5 \mathrm{~nm}$ with a FWHM of $4.8 \AA$ probing the upper photosphere, (3) a continuum channel at $300 \mathrm{~nm}$, (4) a narrow-band $\mathrm{Ca}$ II $\mathrm{H}$ channel with a FHWM of $1.1 \AA$, and (5) a broader Ca II H channel with a FHWM of $1.8 \AA$. The latter two are centered at $397.6 \mathrm{~nm}$ and probe the mid/lower chromosphere.

The optical path of SUFI is incorporated into the Image Stabilization and Light Distribution (ISLiD) unit that splits the different wavelengths between the different instruments using a dichroic beam-splitter (see Figure 1.11). The image stabilization and pointing is carried out by the Correlation tracker and Wavefront Sensor (CWS Berkefeld et al. 2011). For more details please see Section 2 of Gandorfer et al. (2011).

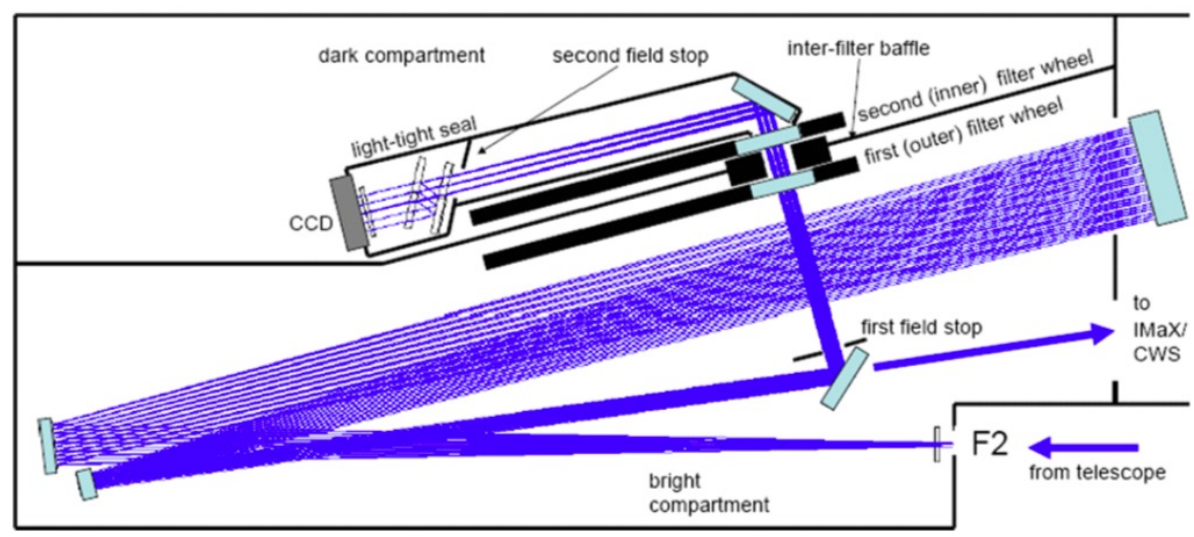

Figure 1.11: Optical layout of SUFI. The layout is optimized for low parasitic light. The two plates in front of the CCD show the phase diversity plates described in Sect. 1.3. Taken from Gandorfer et al. (2011).

The light is collected by a fast read out camera with $2048 \times 2048$ pixels with a sampling of $0.0207 \mathrm{arcsec} / \mathrm{pixel}(\approx 15 \mathrm{~km})$. The exposure time is controlled by a mechanical shutter that compensates not only for the variations of the light level difference between the disk center and the limb, but also matches exposure to the used filter and thus optimizes the contrast of the target.

Despite the near-ideal observing conditions in the stratosphere, some residual wavefront deformations are still expected, caused by unavoidable imperfections in the optical system. To correct this the SUFI camera is divided into two parts - both observing the same field-of-view (FOV) - one in focus and the other one slightly out of focus. This allows to correct the wavefront using phase diversity (PD) techniques that we describe in more detail in Sect. 1.3.2. The resulting FOV in the plane of the sky is approximately $15 \times 40$ arcsec.

\subsubsection{Data reduction}

\subsubsection{Phase-diversity technique}

Phase diversity or PD techniques (Gonsalves and Chidlaw 1979; Löfdahl and Scharmer 1994) are based on the simultaneous recording of two images of the same solar scene, 
one with an unknown wave-front deformation and a second one with the same unknown wave-front deformation plus a known aberration. This can be expressed in the set of the two following equations (Löfdahl and Scharmer 1994):

$$
d_{0}=f * t_{0}+n_{0}
$$

and

$$
d_{k}=f * t_{k}+n_{k}
$$

where $f$ is the real solar scene, $d_{0}$ and $d_{k}$ are the image with the unknown and the image with unknown plus known deformations respectively, $t_{0}$ and $t_{k}$ are the correspondent point spread functions, and $n_{0}$ and $n_{k}$ represent the noise of the measurement. The symbol $*$ indicates the convolution.

The goal of this method is to solve these equations to obtain the real solar scene $f$ by estimating the unknown wave-front deformation. The main limitation of the method comes from the noise represented by $n$, resulting in small-scale errors in the wave-front estimation. These errors lead to reconstruction artifacts in the restored images.

The SUFI approach to this method (for more details see Hirzberger et al. 2011) was to record the two images on the same detector, one in focus and the other out of focus by approximately half a wave. This is achieved by splitting the light beam by two inclined parallel glass plates located in the secondary focus. The special coating of these plates allows half of the light to pass through them without reflections and the other half to be reflected between the plates deviating the beam and increasing the optical path length leading to the desired defocus of half a wave in the image.

The PD reconstruction is the last step in the SUFI data reduction process. It is applied after flat-fielding and dark current correction. As a result we then get 3 levels of data in addition to the raw data:

- level-0: raw data, including both focused and defocused images,

- level-1: flat-field and dark-current corrected data,

- level-2: PD reconstructed data using an individual PSF estimation for each frame, and

- level-3: PD reconstructed data using a PSF estimation averaged in time over the whole observing sequence.

\subsubsection{Multi Object Multi Frame Blind Deconvolution technique}

After all the standard procedures of data reduction, other post-processing numerical techniques can be used to further increase the quality of the dataset. These techniques are usual because they correct aberrations of higher order than the Adaptive Optics (AO) and PD system can (Berkefeld 2007; Rimmele and Marino 2011)

In solar physics two techniques are commonly used for this further image improvement: the speckle reconstruction technique (Speckle Imaging, SI, Keller and von der Lühe 1992) and the Multi Object Multi Frame Blind Deconvolution technique (MOMFBD, Löfdahl 2002; van Noort et al. 2005). Both methods have their strengths and weaknesses and the choice depends on the specific application. In the case of SI the main disadvantages are 
the impossibility to estimate the degradation function, the high number of measurements needed (around 100), and performance problems if the AO is active. The MOMFBD main disadvantages are related to the computational cost and non-idela performance in low contrast images.

In this thesis we use only the second method, MOMFBD, and more precisely just the multi-frame blind deconvolution (MFBD; van Noort et al. 2005) because the reconstruction was just preformed using only one filter, and therefore only one object exists.

To apply this method some requirements need to be fulfilled. The first is, the model assumes that the wavefront degradation is linear and space-invariant. The second is that multiple images of the same object are required, and the solar scene during the recording of these images must be time invariant, i.e., the temporal evolution of the solar structures must be much slower than the time needed to record the images. This is a result of the fact that the true solar scene and the degradation function are unknown, and therefore several independent measurements of the same unchanged object are required. For a typical wideband filter at least 5 frames are required. To ensure that the space invariance is valid, the FOV needs to be smaller than the so called seeing isoplanatic patch. This implies that for lager FOVs the image needs to be divided in smaller regions which have to be restored independently.

The MFBD technique uses a set of many aberrated and noisy measurements to retrieve the unchanged real solar scene. It is described by (following van Noort et al. 2005):

$$
I_{i}=H_{a t m+t e l}^{i} I_{\odot}+N_{i}
$$

where the $I_{i}$ represent the aberrated, independent measurements, the $H_{a t m+t e l}^{i}$ represent the optical transfer function $(\mathrm{OTF}), I_{\odot}$ the unabberated solar scene, and $N_{i}$ is the Fourier transform of the noise. In the case of SUNRISE, due to the almost seeing free conditions, the only significant contribution to the OTF comes from the telescope itself.

The solution to this problem is archived by minimizing the error matrix $(L)$ using the least square differences between the recorded $I_{i}$ and the estimated ones (Paxman et al. 1996):

$$
L(k)=\sum_{k}\left(\sum_{i=0}^{N_{\text {frames }}-1}\left|I_{i}\right|^{2}-\frac{\left|\sum_{i=0}^{N_{\text {frames }}-1} I_{i}^{*} \hat{H}_{\text {atm }+ \text { tel }}^{i}\right|^{2}}{\sum_{i=0}^{N_{\text {frames }}-1}\left|\hat{H}_{\text {atm }+ \text { tel }}^{i}\right|^{2}+\gamma}\right),
$$

where the coefficients $k=\left\{k_{i, m}\right\}$ are the expansion coefficients from the seeing-aberrated wavefronts $\delta_{i}$ (see equation 1.13), $\hat{H}_{a t m+t e l}^{i}$ are the estimated OTFs and $\gamma$ is the regularization term of a simple Wiener deconvolution (Saha 2007).

The seeing-aberrated wavefront is described by

$$
\delta_{i}=\delta_{0, i}+\sum_{m=0}^{N_{\text {modes }}-1} k_{i, m} \Omega_{i, m}
$$

were $N_{\text {modes }}$ represents the number of modes, $\Omega_{i, m}$ are the tilts terms (that takes into account the deviations in the beam direction) and atmospheric Karhunen-Loéve modes represented by Zernike polynomials, and $\delta_{0, i}$ describe possible know phase differences.

In a final step the so-retrieved seeing-aberrated wavefront is used to compute the OTF and to retrieve $I_{\odot}$, the true solar scenery. 


\section{Paper I- Morphological properties of Ca II H slender fibrils ${ }^{1}$}

\subsection{Abstract}

We use seeing-free high spatial resolution Ca II H data obtained by the SUNRISE observatory to determine properties of slender fibrils in the lower solar chromosphere. In this work we use intensity images taken with the SUFI instrument in the Ca II $\mathrm{H}$ line during the second scientific flight of the SUNRISE observatory to identify and track elongated bright structures. After the identification, we analyze theses structures in order to extract their morphological properties. We identify 598 slender Ca II H fibrils (SCFs) with an average width of around $180 \mathrm{~km}$, a length between 500 and $4000 \mathrm{~km}$, an average lifetime of $\approx 400 \mathrm{~s}$, and an average curvature of $0.002 \mathrm{arcsec}^{-1}$. The maximum lifetime of the SCFs within our time series of 57 minutes is $\approx 2000 \mathrm{~s}$. We discuss similarities and differences of the SCFs with other small-scale, chromospheric structures such as spicules of type I and II, or Ca II K fibrils.

\subsection{Introduction}

Large parts of the solar surface are littered with small-scale fibrils, loops, and jets, connecting the photosphere to the chromospheric layers. These structures, seen in radiation, are thought to follow magnetic field lines (e.g., Jafarzadeh et al. 2017) anchored in photospheric magnetic flux concentrations, or in the weaker internetwork elements (Wiegelmann et al. 2010). Except for regions with large magnetic flux concentrations, such as sunspots or large pores, these structures play an important role in transporting the energy from the solar surface to the chromosphere and to the corona, either as a channel for the propagation of waves (e.g., van Ballegooijen et al. 2011), or as the location for small-scale reconnection events (Gold 1964; Parker 1972). Observations in the Ca II H and K lines at high spatial resolution (e.g., from the Swedish Solar Telescope, SST, Pietarila et al. 2009), and under seeing-free, stable conditions using the Hinode space observatory, shed new light on these small-scale structures, e.g., leading to the discovery of a new type of spicule (type-II, de Pontieu et al. 2007; Pereira et al. 2012).

\footnotetext{
${ }^{1}$ This chapter reproduces the article Morphological properties of Ca II H slender fibrils by R. Gafeira, A. Lagg, S. K. Solanki, S. Jafarzadeh, M. van Noort, P. Barthol, J. Blanco Rodríguez, J. C. del Toro Iniesta, A. Gandorfer, L. Gizon, J. Hirzberger, M. Knölker, D. Orozco Suárez, T. L. Riethmüller, and W. Schmidt, published in ApJS, 229, 2017, DOI 10.3847/1538-4365/229/1/6. Reproduced with permission of ApJS
} 
The unique observational conditions provided by the SUNRISE balloon-borne solar observatory (Barthol et al. 2011; Solanki et al. 2017) allow us to observe the solar chromosphere in the core of $\mathrm{Ca}$ II $\mathrm{H}$ at constantly high temporal and spatial resolution, without the influence of seeing. This has given us the possibility to look at the structures present in the lower chromosphere at a level of detail not achieved before. Of special interest in this work are the so-called slender Ca II H fibrils (SCFs): similar to spicules or chromospheric jets, these ubiquitous features outline the magnetic field in the lower chromosphere and offer the possibility of gaining insight into the physical processes in this layer of the solar atmosphere (Pietarila et al. 2009; Wöger et al. 2009; Jafarzadeh et al. 2017).

In this work, we develop a technique for the automatic detection of SCFs (Sect. 2.4) enabling us to investigate their statistical properties. We discuss the lifetime, width, length, curvature, and the temporal evolution of brightenings within the individual SCFs (Sect. 2.5), and compare these morphological properties to similar, small-scale structures observed with Hinode and the SST (Sect. 2.6).

\subsection{Data}

The observations on which this study is based were taken by the SUNRISE balloon-borne solar observatory (Solanki et al. 2010; Barthol et al. 2011; Berkefeld et al. 2011; Gandorfer et al. 2011; Martínez Pillet et al. 2011) during its second science flight (Solanki et al. 2017) in 2013 June, referred to as SUNRISE II. The data set used was recorded from 2013 June 12 at 23:39 UT to 2013 June 13 at 00:38 UT, and covers part of the active region NOAA 11768 including most of the following polarity, the polarity inversion line, and also an emerging flux region lying between the two opposite polarity regions. At the time it was observed the active region was still young and developing and was located at $\mu=\cos \theta=0.93$, where $\theta$ is the heliocentric angle. The heliocentric coordinates of the center of the SUNRISE Filter Imager (SUFI) field-of-view (FOV) were $x=508^{\prime \prime}$, $y=-274^{\prime \prime}$. The one hour long time series is composed of a total of 490 images taken using the SUFI (Gandorfer et al. 2011) in three wavelength bands, the Ca II H 3968 A line using a narrow-brand filter with a full width at half maximum (FWHM) of $1.1 \AA$. The integration time of each image was $500 \mathrm{~ms}$. A broader Ca II $\mathrm{H}$ channel with a FWHM of $1.8 \AA$ recorded images with an integration time of $100 \mathrm{~ms}$, and a broad-band channel centered on $3000 \AA$, with a FWHM of $50 \AA$, delivered continuum images with an integration time of $500 \mathrm{~ms}$. The data were reconstructed using the multi-frame blind deconvolution (MFBD, van Noort et al. 2005) technique to account for the image degradation by the telescope. After the MFBD reconstruction, the data had a spatial resolution close to the diffraction limit of the SUNRISE telescope, which is at the wavelength of the Ca II H line, approximately $70 \mathrm{~km}$. The cadence (i.e. the time between two consecutive images at the same wavelength) was $7 \mathrm{~s}$. The data set, the data reduction, and reconstruction are described in detail by Solanki et al. (2017).

\subsection{Fibril detection and tracking}

The main goal of this work is to determine the basic morphological properties of the SCFs. To this end we must first identify and track the bright elongated structures that can be seen 


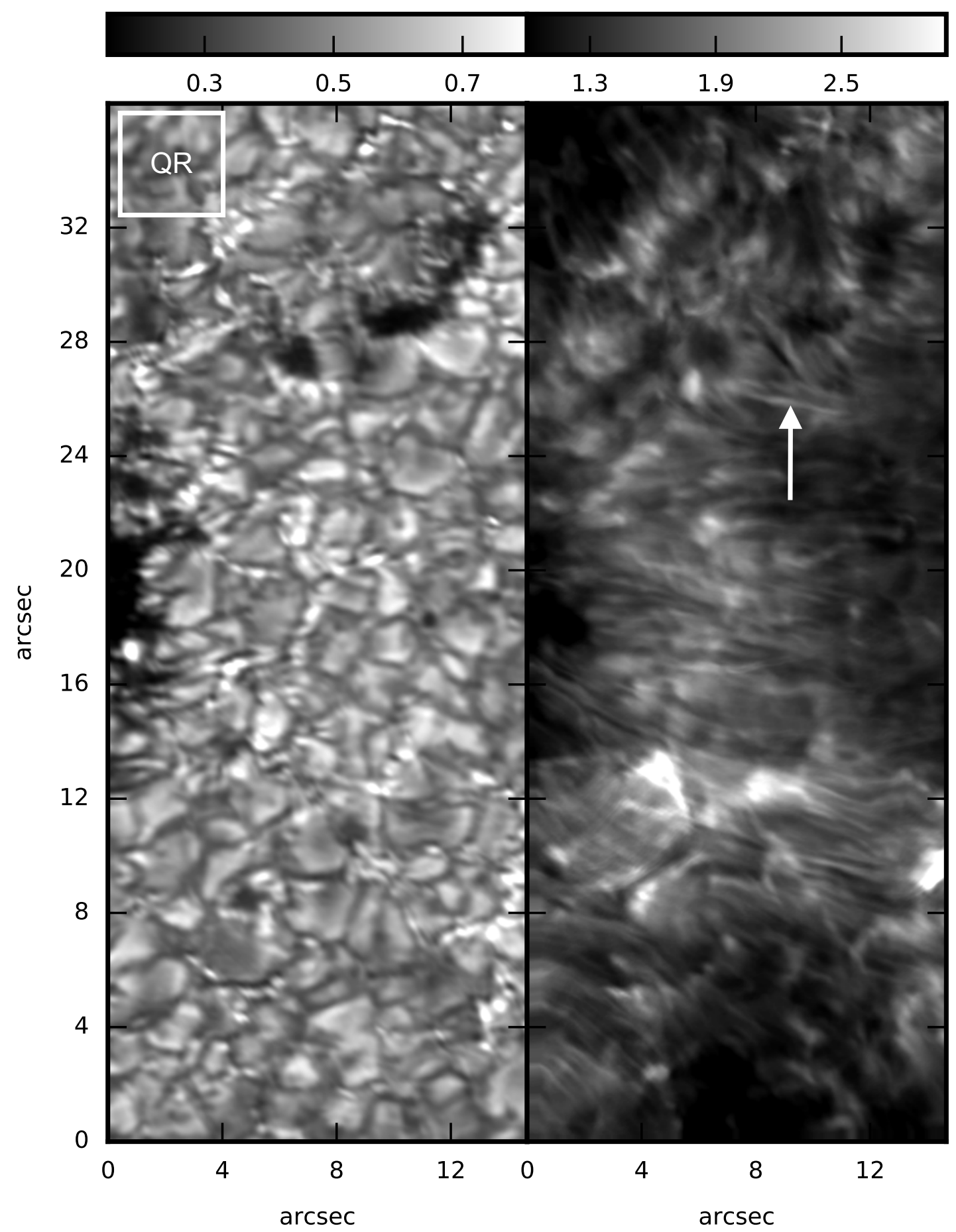

Figure 2.1: (A) Continuum image recorded on 2013 June 13 at 00:31 with the $3000 \AA$ continuum filter, (B) cotemporal, coaligned $\mathrm{Ca}$ II $\mathrm{H}$ image, (C) binary mask resulting from the fibril identification method, and (D) superposition of the Ca II H image and the identified fibrils. The dashed red line on image A indicates the position of the slit used in Figure 2.4. The white arrow on image B points to a sample fibril that is shown in greater detail in Figure 2.2. 
in Figure 2.1 B. To perform this identification we apply a series of image processing and contrast enhancement techniques. We start by subtracting a boxcar-averaged version of the original image from itself to remove the low frequencies and to enhance the structure with the typical dimensions of the fibrils. The size of the boxcar window is set to 20 pixels, corresponding to approximately $0^{\prime \prime} .4$. Then, we apply a sharpening filter using the UNSHARP_MASK function from the Interactive Data Language (IDL, Exelis Visual Information Solutions, Boulder, CO) to increase the global image contrast. This unsharp mask involves the following steps: (i) the original image is smoothed with a Gaussian filter having a width of 10 pixels; (ii) this smoothed image is then subtracted from the original image; and (iii) the resulting difference image is again added to the original image. In a next step, an adaptive histogram equalization is performed, described in detail by Pizer et al. (1987), again using the implementation in IDL (version 8.3) with the standard parameter settings to further increase the contrast. Finally, we apply a boxcar smoothing with a width of 3 pixels to remove frequencies beyond the spatial resolution of SUNRISE, introduced by the steps described above.

The resulting contrast-enhanced images highlight most of the fibrils very well and allow the application of a binary mask with a threshold of $50 \%$ of its maximum intensity, separating the fibrils from their surroundings. The same threshold was used for the entire FOV of SUFI and for all frames in the time series, ensuring an unbiased determination of the length, width and shape of the identified fibrils.

Finally, we exclude all detected regions smaller than 200 pixels in area (corresponding to $\approx 0.1 \operatorname{arcsec}^{2}$ ), which is approximately the area of a circle corresponding to a spatial resolution element close to the diffraction limit. An example of such a binary mask resulting from this process is shown in Figure 2.1 C.

The next step is to track individual fibrils in time to analyze their evolution. For this task we take six consecutive frames and identify those regions where the binary mask marks the presence of a fibril in at least 10 pixels at the same image position in at least five out of a total of six consecutive frames. After this, we assign each fibril a tracking index. Then we advance one frame and repeat the same analysis, checking whether each newly identified fibril already has a tracking index. If so, it is counted as the temporal continuation of the fibril from the previous frame; if not, it is counted as a new fibril.

This method ensures, with a high level of confidence, that we catch the temporal evolution of individual fibrils. The method works very well for nicely separated fibrils, but it has some limitations in two special cases: (i) if a significant number of other fibrils cross the identified fibril, the binary mask will show either interruptions or the crossing fibrils are counted toward the identified fibril, and (ii) if the identified fibril has a high transverse velocity (i.e., perpendicular to the fibril axis), it will move out of the detection window in the next frame. The latter effect is particularly important in the regions of magnetic flux emergence (near $x=12^{\prime \prime}, y=9^{\prime \prime}$ ) where the fibrils are much more dynamic than in the rest of the SUFI FOV.

Due to the large density of fibrils, about $80 \%$ of those detected fibrils suffer from the presence of crossing or very close neighboring fibrils. The identified fibril and those crossing it are often misidentified as a single structure with a complex, frayed shape instead of the expected linear, elongated one identified by the white arrow in Figure 2.1. If such a complex shape does exist over several consecutive frames, a correct fibril identification using a binary mask is nearly impossible. 
To address this problem we introduce the concept of a fibril's reference backbone, which we define as the second-order polynomial best fitting the long axis of the fibril and therefore marking the ridge of maximum brightness. It is computed by first reducing the fibril in every frame of the time series of $\mathrm{Ca}$ II $\mathrm{H}$ images to a shape that is a single pixel in width. Each such pixel is equidistant from the fibril's lateral boundaries (which are the locations where the brightness drops below the threshold). The set of all such (single) pixels constitute the fibril's backbone. Afterwards, the fibrils of the individual frames are fitted with a second-order polynomial. This polynomial is extended by the average width of this fibril (see Sect. 2.5.2), on both end points of the fibril to compensate for the shortening due to the reduction of the fibril to a single pixel line. The resulting curve we define as the reference backbone. Fibrils of complex shape, often produced by intersecting fibrils or by not well separated fibrils, are usually poorly fitted ( $\chi^{2}$ values are higher than 6), and are excluded from this averaging. An example of this process is illustrated in Figure 2.2, where we show one identified fibril and its temporal evolution (left panels) and the overplotted fibril backbone (black line, right panels). The gray shaded area around the skeleton displays the width of the fibril, computed in every single frame using the method described inSect. 2.5.2.

The fibril is well identified in all six plotted time steps. However, its length may well be underestimated, as the detection method often fails to follow the fibril to its very end, as can be seen by eye. Fibrils often become too faint near their ends to be reliably identified by our method. Being faint, they are also susceptible to interference from background structure and crossings by other fibrils. We have therefore not attempted to extend the lengths of the individual fibrils. Instead, we consider that the fibrils that we have identified are often in fact fibril fragments (see Pietarila et al. 2009), i.e., parts of longer fibrils. For brevity we will continue to call them fibrils (or SCFs) in the following, although in many cases we may be discussing only parts of the full fibrils.

We obtain a total of 598 fibrils in the SUFI FOV over the entire time series. One snapshot with the identified fibrils overlayed on top of the corresponding $\mathrm{Ca}$ II H image is presented in panel D of Figure 2.1.

\subsection{Fibril morphology}

The result of the fibril tracking allows for a statistical analysis of the SCFs and to compute several of their morphological properties. In this section we present the results obtained for lifetime, length, width, and curvature of SCFs extracted using information obtained from the detection and tracking methods.

\subsubsection{Lifetime}

Visual inspection of the movie created from the time series of SUFI images (see online material, sufi.mp 4) reveals the highly dynamical nature of the SCFs. The identification of the individual fibrils over multiple time steps allows us to produce a lifetime histogram with a lower limit of $35 \mathrm{~s}$ (corresponding to five consecutive frames, which is the minimum length of time we require for a fibril to survive to be identified as such) and an upper limit given by the length of the time series (57 minutes). Only fibrils with a defined start- 


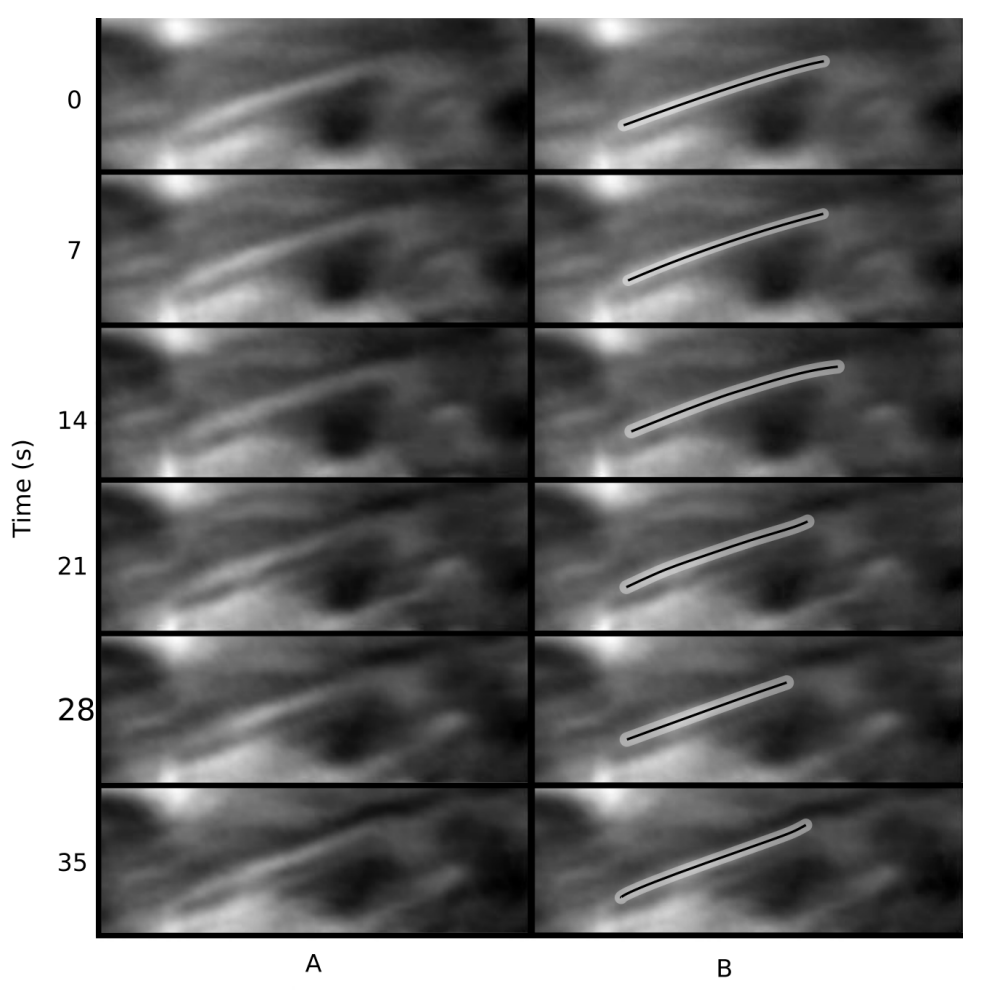

Figure 2.2: Example of the temporal evolution of a tracked fibril. The left panels show the original $\mathrm{Ca}$ II $\mathrm{H}$ images, representing the temporal evolution of a fibril identified over a set of six consecutive SUFI frames. The right panels show the same images, overplotted with the result of the detection and tracking method described in the main text. The black lines represent the individual backbones and the gray regions indicate the width of the fibril computed using the method explained in Sect. 2.5.2.

and end-time are included in the histogram, i.e., fibrils that are observed in the first or the last frame of the series are excluded from this analysis.

This lifetime histogram is presented in Figure 2.3 (all histograms shown in this paper are normalized so that they have a total area equal to 1). It shows a clear exponential decay with increasing lifetime at a decay rate of $2.5 \times 10^{-2} s^{-1}$ (red line). About $80.3 \%$ of the fibrils have lifetimes between $35 \mathrm{~s}$ (i.e., the lower threshold) and $100 \mathrm{~s}$, and only $1.6 \%$ live longer than $300 \mathrm{~s}$ (see also Table 2.1). It must be noted that these lifetimes are likely underestimated for multiple reasons. By considering only fibrils that are born and die in the course of the observed time series, we bias the histogram toward shorterlived fibrils due to the finite length of the time series. Changes in either the fibril or its background or neighboring fibrils can also cause its lifetime to be underestimated. Changes in the brightness or position of the fibril can cause the tracking algorithm to lose it, thus curtailing its apparent lifetime. Similarly, variations in the overall brightness and/or quality of the image may cause an apparent disappearance and reappearance of the same fibril, which is then counted as two individual fibrils, both of them with a shorter apparent lifetime. In addition, fibrils clearly visible on the dark background of the outer parts of the pore and its immediate surroundings become hard to follow above the bright magnetic concentrations in the right part of the image. Also, there appear to be weak, 


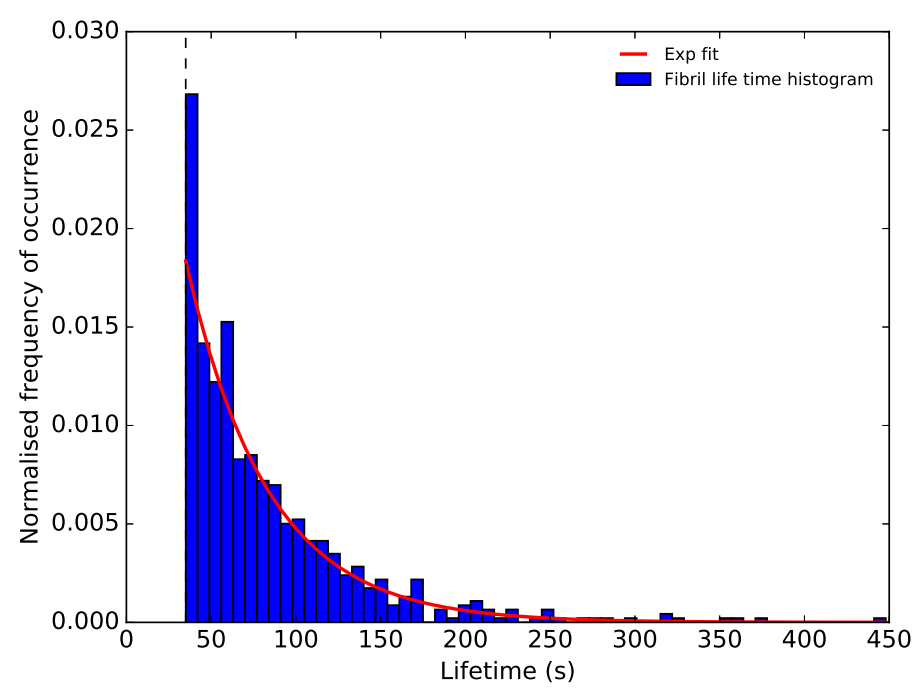

Figure 2.3: Fibril lifetime distribution and an exponential fit to the observed distribution (red line). Plotted is the frequency of occurrence of fibrils per lifetime bin versus the lifetime. Only fibrils with a minimum lifetime of $35 \mathrm{~s}$ are included.

short, and strongly inclined fibrils connecting bipolar regions in the right part of the figure that are directed from the NE to the SW, which cross the longer mainly E-W directed fibrils. Similarly, in the upper half of the figure, fibrils emanating from the large pore often cross other fibrils pointing in different directions. Hence the automated identification catches only individual relatively undisturbed fragments of fibrils.

To investigate this effect, introduced by the tracking method, we analyzed the position of the fibrils in a space-time diagram. Figure 2.4 shows the temporal evolution of fibrils along an artificial slit at a fixed $x$-position, $x=8^{\prime \prime}$, indicated by the red, dashed line in Figure 2.1 A. The slit stays fixed at this position throughout the whole time series. Plotted is a binary mask, with the presence of (identified) fibrils indicated in blue. In many cases, the blue patches look like an intermittent, nearly horizontal line, indicating that this particular fibril first disappears and then reappears at almost the same location. For better visibility, one such intermittent line is highlighted in red. Most of the longer interruptions indicate a true darkening (or complete disappearance) of the fibril, with a later reappearance within the same magnetic flux tube or set of field lines, which obviously has a lifetime longer than the fibril itself. Also, some of the brightness variations leading to interruptions of fibrils in a space-time plot may be related to the sausage-mode waves identified in the fibrils by Gafeira et al. (2017a).

To identify the intermittent fibrils, exemplified by the one marked in red in the spacetime diagram presented in Figure 2.4, we use exactly the same approach as described in Sect. 2.4, but with different parameter settings, allowing us bridge the longer temporal gaps. In Sect. 2.4, we required that 10 common pixels must exist in at least five out of six consecutive frames, to ensure that we are really tracing the same fibril. Here, we relax this condition and require that 10 common pixels must exist in only two out of 16 frames. This closes most of the temporal gaps visible in Figure 2.4, at the expense of an increased likelihood of catching another fibril, which moved into the same position as the previous 


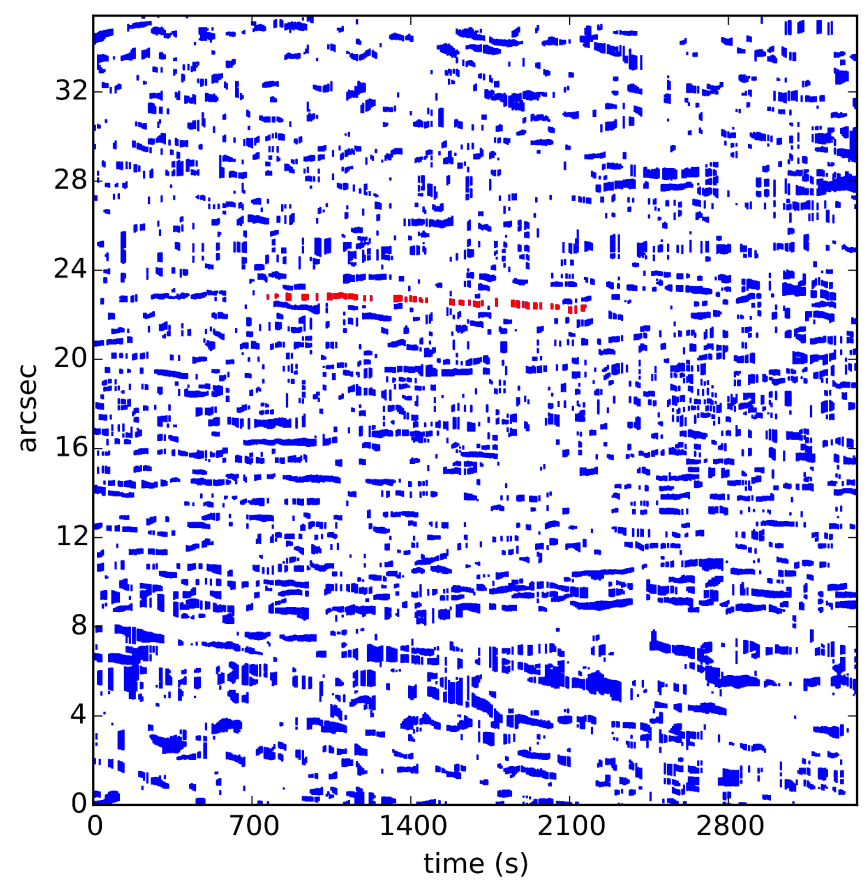

Figure 2.4: Space-time diagram of the presence of fibrils along an artificial slit indicated by the red dashed line in Figure 2.1 A. One fibril is marked in red to exemplify its intermittent appearance.

fibril within $\leq 105 \mathrm{~s}$ (15 frames).

The so-determined lifetime is still underestimated due to the maximum allowed gap length of 15 frames, and the finite length of the time series, as longer-lived fibrils are more likely excluded because they were alive either at the beginning or the end of the time series. The latter effect can be corrected following Danilovic et al. (2010): we multiply the frequency of occurrence of fibrils that live for $m$ frames by the factor $(n-2) /(n-1-m)$, where $n$ is the total number of frames in the time series. This correction has been taken into account in Figure 2.5. The average lifetime of the fibrils obtained after all these corrections is Figure 2.5 is $446 \mathrm{~s}$. Other parameters describing the lifetime distribution are listed in Table 2.1.

We then compared this lifetime distribution with that from the automatic tracking method. The new distribution, presented in Figure 2.5, now shows a maximum at a lifetime of $\approx 150-300 \mathrm{~s}$. More than $58 \%$ of the fibrils have lifetimes longer than $300 \mathrm{~s} ; 8.5 \%$ even live longer than 15 minutes. The red line indicates an exponential fit to the tail of the distribution (i.e., to the distribution of lifetimes longer than $400 \mathrm{~s}$ ) and the green line indicates a log-normal fit to the entire distribution. The decay rate is now found to be $3.0 \times 10^{-3} \mathrm{~s}^{-1}$, meaning that the distribution drops off nearly an order of magnitude slower than before the correction of the lifetimes for the intermittency. Obviously, the magnetic structure (possibly some kind of flux tube) housing the SCFs is considerably longer-lived than the brightenings in the $\mathrm{CaIIH}$ line. It could also be that the source of the brightening (located, e.g., in the photosphere) is intermittent, causing a bright fibril to 
appear equally intermittently.

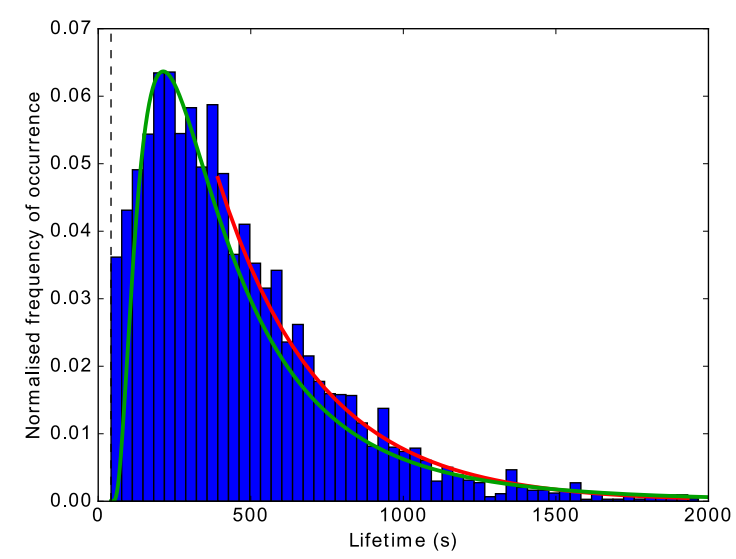

Figure 2.5: Fibril lifetime distribution taking into account the intermittency of the detected fibrils. The red line indicates an exponential fit for fibrils with a lifetime $\geq 400 \mathrm{~s}$. The green line indicates the respective log-normal fit.

\subsubsection{Width}

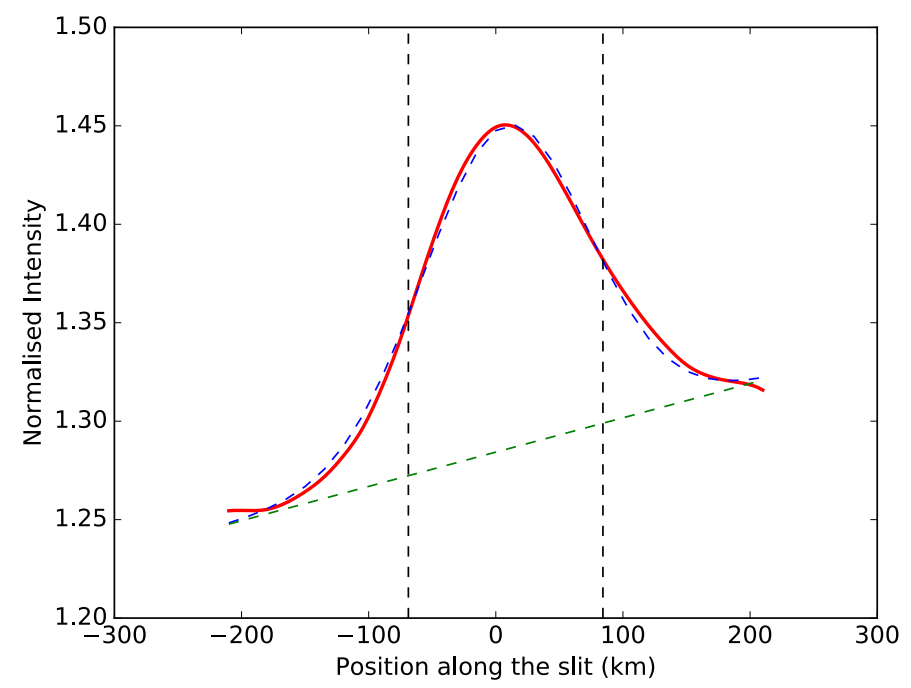

Figure 2.6: Example for the determination of the fibril width by fitting a superposition of a Gaussian and a linear function (dashed blue line) to the brightness variation (red line) perpendicular to the fibril backbone. The linear function (dashed green line) takes into account the variation of the background brightness. The width of the fibril, indicated by the vertical dashed lines, is the FWHM of the Gaussian.

The automatic detection method delivering the backbone of the fibrils facilitates the computation of the widths of the SCFs. This computation, illustrated in Figure 2.6, is 
done by first selecting the position of the maximum intensity of a fibril along a virtual slit, with a length of $0^{\prime \prime} .6$, oriented perpendicularly to the backbone of the fibril and placed at the center of the fibril's backbone. Then we fit a weighted Gaussian function plus a linear background to the intensity on the slit. Points closer to the maximum intensity are given larger weights, in order to avoid that the fit is negatively influenced by close neighboring fibrils. We then take the FWHM of the Gaussian as a measure of the fibril's width. To further minimize the influence of neighboring or intersecting fibrils we require that the side flanks of the fibril to be clearly visible and the linear background brightness variation to have a slope smaller than 0.002 per $\mathrm{km}$ in units of the normalized intensity.

The above procedure is repeated for all fibrils in each frame to produce the histogram presented in Figure 2.7. This distribution of fibril widths is reasonably well represented by a Gaussian function (red line) with a mean width of $\approx 182 \mathrm{~km}$ (see Table 2.1 for the other fit parameters of this Gaussian). The narrowest fibril was determined to have a width slightly smaller than $100 \mathrm{~km}$, which is above the spatial resolution of SUNRISE of around $70 \mathrm{~km}$ at the wavelength of CaII H. This fact might suggest that $\approx 100 \mathrm{~km}$ is a lower limit for the width of solar fibrils, and that SUNRISE is actually resolving the fibrils. However, we are aware of the fact that the determination of the fibril width is not without problems. The fibrils have to be identified against a relatively bright photospheric background contamination resulting from the broad transmission profile of the $\mathrm{Ca}$ II $\mathrm{H}$ filter used in SUFI. Additionally, the width determination method, based on the fitting of a Gaussian function, is likely to smear out structures at the diffraction limit of the telescope, resulting in a larger minimum width.

The study of the width of Ca II K fibrils by Pietarila et al. (2009) showed minimum fibril widths of only $70 \mathrm{~km}$, corresponding to the diffraction limit of the SST. Their analysis method was based on a binary mask, which is possibly better suited to determining the minimum widths of the fibrils, but has disadvantages in properly characterizing broader fibrils. When applying our technique to a sample of the fibrils in the data set used by Pietarila et al., we also get $\approx 100 \mathrm{~km}$ for the minimum width of their Ca II K fibrils, in accordance with our SUNRISE/SUFI measurements. We therefore conclude that the minimum width of our Ca II fibrils is likely to be set by the spatial resolution of the SUNRISE telescope.

\subsubsection{Length}

An important parameter returned by the automatic tracking method is the length of the detected SCFs, which we define as the length of the reference backbone as described in Sect. 2.4, plus the average of the widths computed in Sect. 2.5.2 at both end points. This addition is necessary to compensate for the effect when reducing the fibril to the singlepixel backbone, which reduces the backbone length on both ends approximately by the width of the fibril. The length of the fibril as determined here would correspond to the distance from end-to-end of the shaded area in each of the right-hand panels of Figure 2.2. Fibrils at the edge of the FOV are excluded from the analysis.

The histogram in Figure 2.8 shows that the detected fibrils are rather short, with a minimum length of $\approx 500 \mathrm{~km}$. The mean length of the fibrils is $\approx 1300 \mathrm{~km}$, and we could not find any fibril longer than $4500 \mathrm{~km}$. The length distribution nicely follows a lognormal distribution, indicated by the red line in Figure 2.8 (for the fit parameters see 


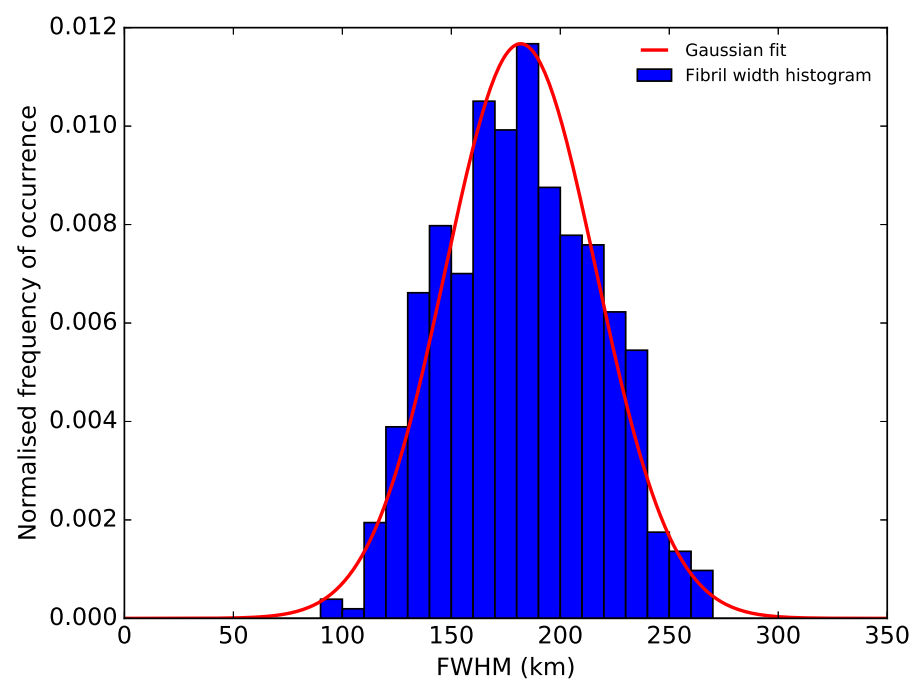

Figure 2.7: Width distribution for each individual fibril detected in all the frames and its respective Gaussian fit (red curve).

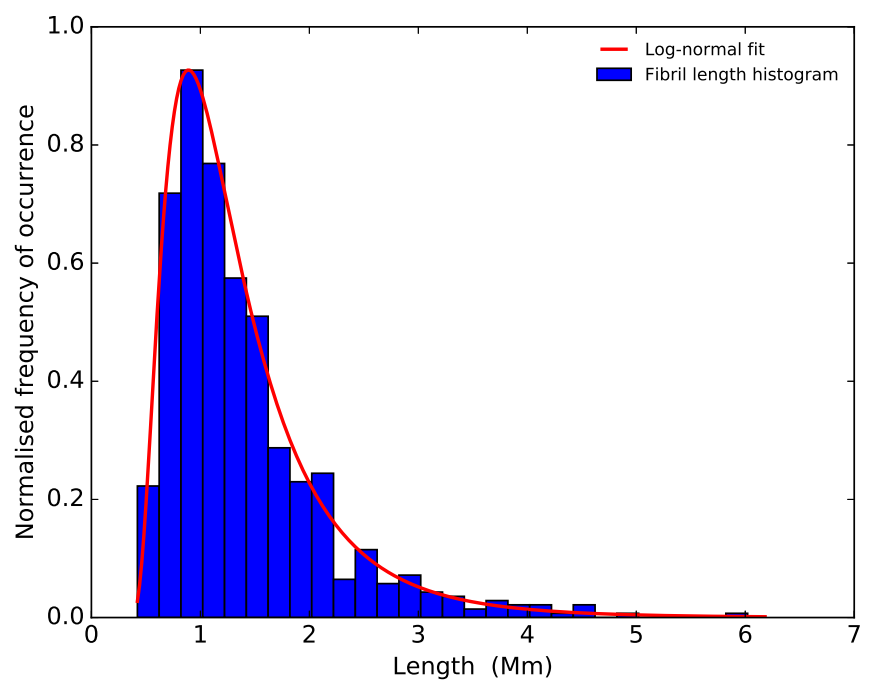

Figure 2.8: Distribution of the backbone length, averaged over the lifetime of the SCFs (blue), and respective log-normal fit (red curve).

Table 2.1).

Just as with the longer-lived fibrils, the longer fibrils are also more likely to be excluded because they touch one of the boundaries of the FOV. We again follow Danilovic et al. (2010) and multiply the frequency of occurrence of fibrils that have a length of $m$ pixels by the factor $(n-2) /(n-1-m)$, where $n$ is the distance from one edge of the frame to the other in the average direction of the fibril. This correction has been taken into account in Figure 2.8. The average length of the fibrils is found to be $1.38 \mathrm{Mm}$. It is worth noting that their length does not correlate very well with their lifetime of the fibrils (the correlation coefficient is only 0.3 ). 
The short length of the fibrils may be an artifact of the automated identification technique, so that we are looking at fragments of fibrils in many cases (see the discussion in Pietarila et al. 2009, and in Sect. 2.4). In the blow-up of the central section of the $\mathrm{Ca}$ II $\mathrm{H}$ image plotted in Figure 2.1 B (data after MFBD reconstruction) many long fibrils are seen, some of them reaching $10^{\prime \prime}$ in length. However, these long fibrils are often interrupted, or cut by other structures, making it hard for our simple identification algorithm to recognize them as a single long entity. Instead, the identification procedure breaks them into shorter fibrils.

There is also a general geometrical separation between the longer and shorter fibrils, at least as seen by eye, in the sense that the longer fibrils are found more in the centrallower part of Figure 2.1 B directed roughly E-W, while the shorter fibrils are located in the upper part of the figure, in particular surrounding the small pores. In addition, the pattern of seemingly very short fibrils, or rather small bright patches in the lower left part of the image, may be produced by crisscrossing longer fibrils.

\subsubsection{Curvature}

The reference backbone of most of the fibrils can be fitted nicely with a second-order polynomial. This has partly to do with the fact that the automatically identified fibrils are rather short, but also because the fibrils are expected to outline loop-like magnetic flux tubes (although rather flat ones; see Jafarzadeh et al. 2017; Wiegelmann et al. 2017): the slanted view at a heliocentric angle of $\theta \approx 22^{\circ}$ makes loop-like structures appear similar to parabolas.

We define the curvature, $\kappa$, of a fibril as the reciprocal value of the radius of the largest circle fitting the fibril tangentially, i.e., the so-called osculating circle. For a secondorder polynomial, $\kappa$ is defined as $2 a$, where $a$ is the second-order polynomial coefficient. The histogram in Figure 2.9 shows the curvature distribution obtained from all reference backbones of the time series. The distribution is nearly symmetric with a slight offset toward a positive curvature, with a mean value of $0.002 \operatorname{arcsec}^{-1}$.

\subsubsection{Brightness variation}

The definition of a fibril backbone simplifies not only the study of the morphological properties discussed in the previous section, it also allows us to visualize the temporal evolution of intensity fluctuations within the fibril itself. This is achieved by putting an artificial mesh onto the reference backbone of the fibril, with the grid lines being parallel and perpendicular to the backbone. The perpendicular grid lines cover a region of $\pm 0.3^{\prime \prime}$ around the backbone; the parallel grid lines have the same length and curvature as the backbone itself. More information on the mesh and gridlines, including an illustration, can be found in Gafeira et al. (2017a).

This mesh allows us to straighten the fibril by interpolating the brightness of the original image to the grid points of the mesh. Since the mesh was defined on the reference backbone of the fibril, we can now nicely follow the temporal brightness variation within the individual fibrils in plots such as the examples presented in Figs. 2.10 and 2.11. These two plots show two different types of brightness variation and propagation along the fibril backbones. In Figure 2.10 the intensity decreases from the center to the endpoints, 


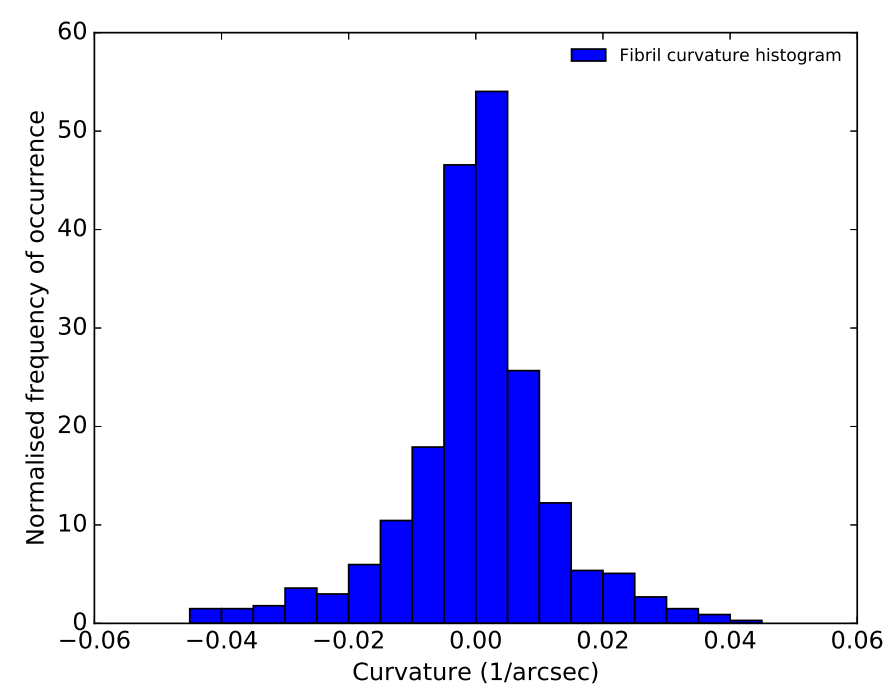

Figure 2.9: Curvature distribution of the fibril's reference backbones.

whereas in Figure 2.11 the intensity decreases from the endpoints of the fibril to its center. After visual inspection of all fibrils we find that the first type is the more common (around $54 \%$ of the the fibrils are of this type), and the second type is observed in $\approx 25 \%$ of the fibrils. For the remaining $\approx 21 \%$ of the fibrils it is difficult to clearly categorize the distribution of the brightness along the fibril axis, with only a very small fraction of those showing a brightening at one endpoint only. We could not find any obvious correlation between the type of the brightening (central or endpoint) with other parameters of the fibrils (length, width, absolute value, lifetime). It can also be seen that the width of the fibrils is not constant along the the length of the fibril. In general, the fibrils are broader at the locations where they are also brighter.

Note that in some cases, a fibril changes from having its brightness peak at its center to having it near its ends. Obviously, the brightness of the fibril varies with time, as can be seen in Figs. 2.10 and 2.11. Gafeira et al. (2017a) have pointed out that these variations are often periodic and occur mainly in (anti-)phase with oscillations in the width of the fibril. They identified these variations with sausage-mode waves. 


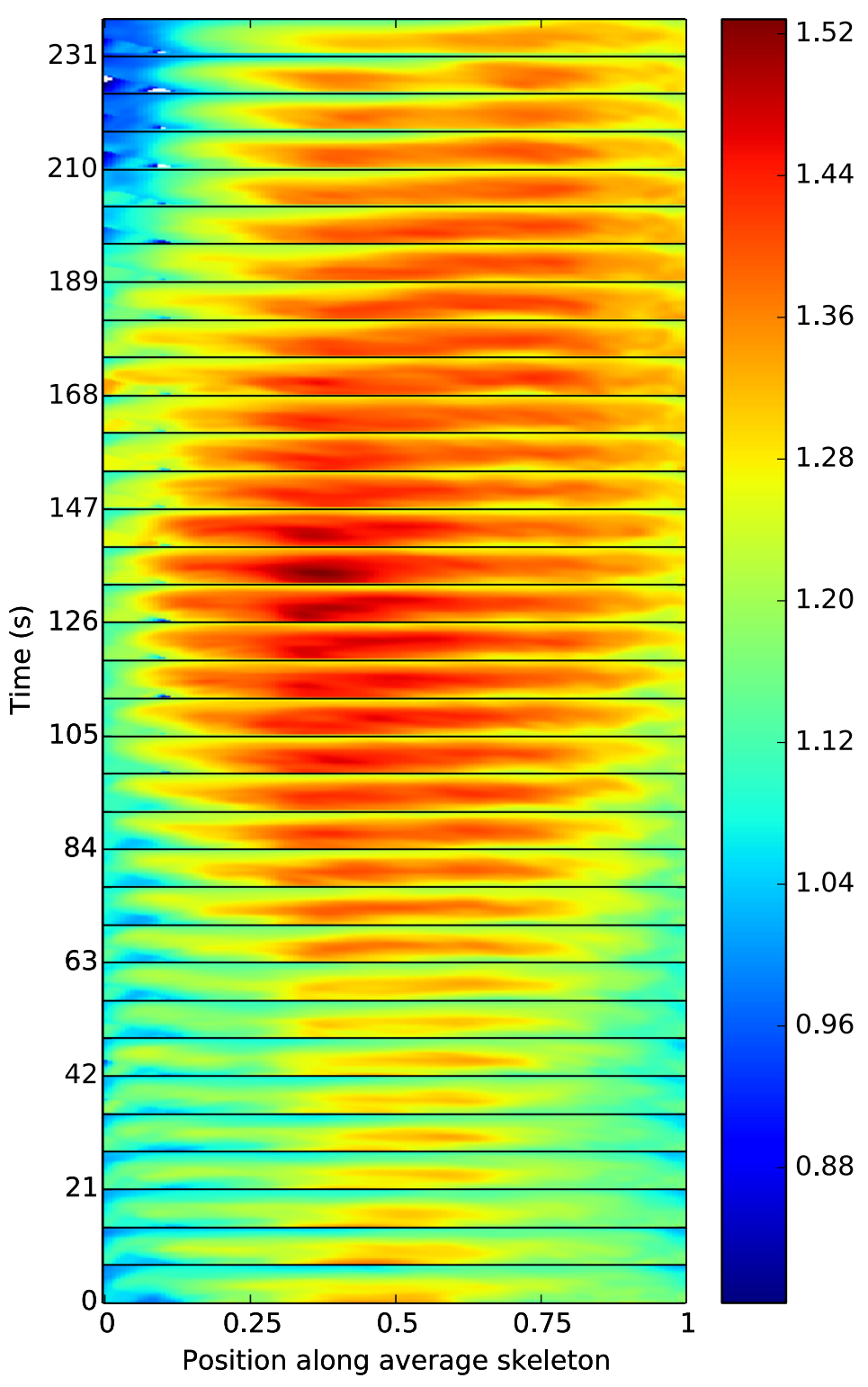

Figure 2.10: Temporal evolution of a SCF with a central brightening. The stacked images show the straightened fibril, with the backbone parallel to the $x$-axis in the center of each bin, surrounded by a $\pm 0.3^{\prime \prime}$ wide area covering the fibril. The individual images, recorded every $7 \mathrm{~s}$, are separated by horizontal black lines. The length of the reference backbone of this fibril is $1620 \mathrm{~km}$. 


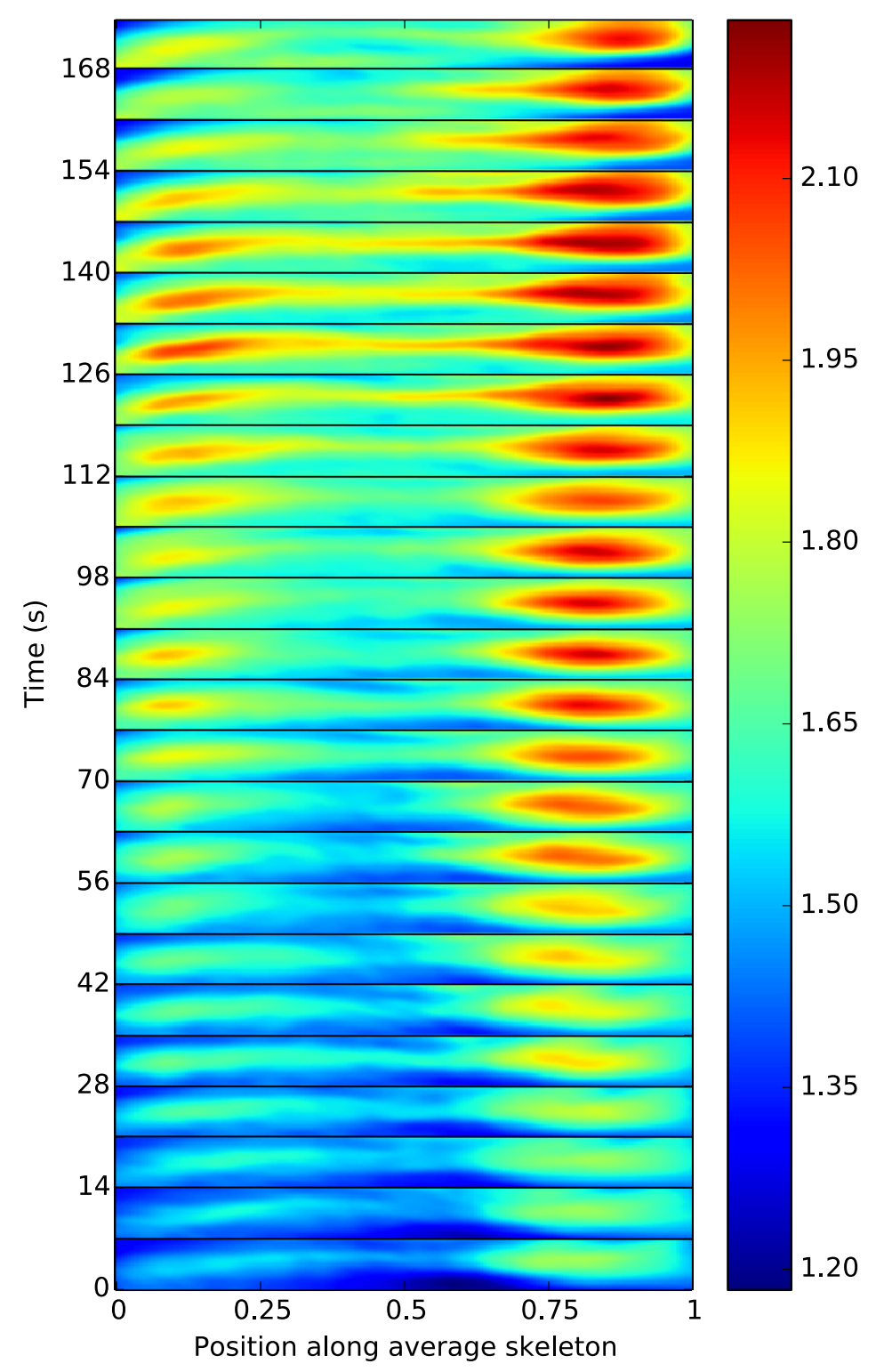

Figure 2.11: Same as Figure 2.10 for a SCF with endpoint brightening. The length of the reference backbone of this fibril is $2800 \mathrm{~km}$. 


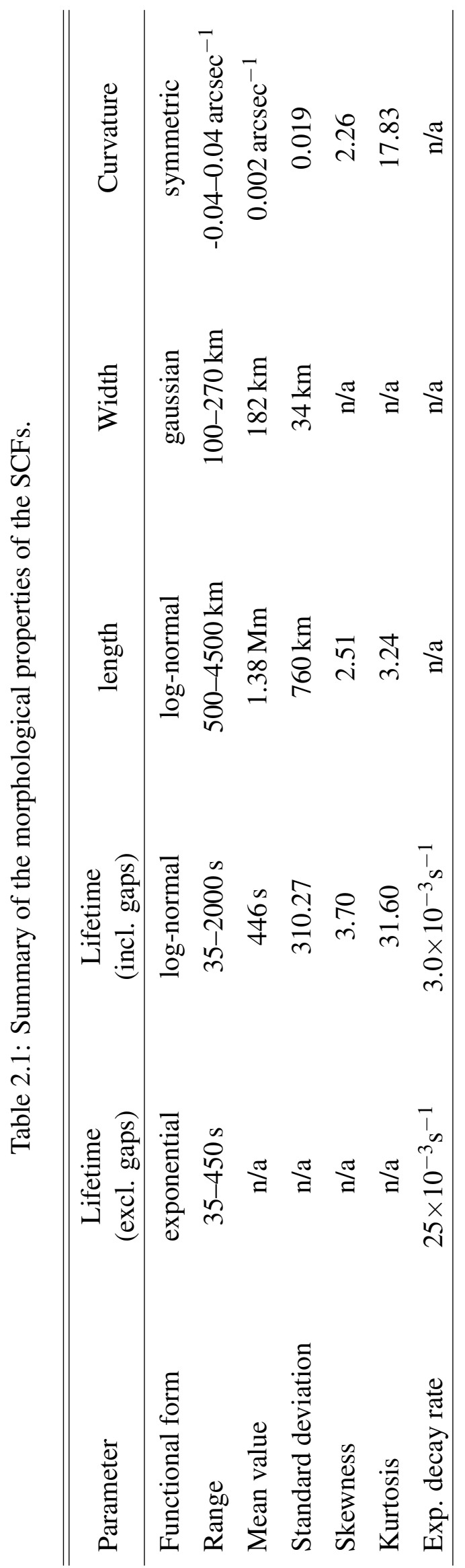




\subsection{Discussion and conclusions}

In this paper we present a first quantitative study of the morphological properties of SCFs aimed at measuring their parameters, such as lifetime, length, width, curvature, and brightness structure. We apply an automatic fibril detection algorithm based on several unsharp masking steps to identify the backbone of the fibrils (i.e., the curve following the central brightness ridge along the long-axis of the SCF). This allows us to identify, measure properties of, and perform a statistical analysis on 598 SCFs. We obtain values for the lifetime between half a minute and half an hour (when taking into account the intermittency in fibril visibility), lengths between 0.5 and $4.5 \mathrm{Mm}$, and widths between 100 and $270 \mathrm{~km}$. The fibrils show a wide distribution of curvatures, with a slight preference for a positive curvature, in agreement with the expected curvatures of low-lying loops observed at a position away from the solar disk center. We also identify two different morphologies of the evolution of the brightness within individual fibrils. In one type the intensity increases starting from the center and moving to the edges, while in the other type the increase in intensity first becomes visible near one or both of the ends and expands from there toward the center of the fibril.

Intersecting fibrils and not well separated fibrils cause problems in the automatic tracking them, mainly leading to a failure in detecting fibrils longer than $4.5 \mathrm{Mm}$, although the visual inspection of the SUFI Ca II H images shows that such fibrils do exist. The contrast between the measured short lengths and the pattern of extended fibrils in parts of the SUFI FOV suggests that the automated technique is identifying relatively undisturbed parts of fibrils as whole fibrils. Between these undisturbed fragments a given long fibril may be intersected by other fibrils, or disturbed by underlying bright points, reversed granulation, and acoustic grains. This complex picture may arise because at the height of the SCFs, the Ca II H line may not be entirely optically thick, so that radiation from below can pass through possibly multiple layers of fibrils. This may have to do with the $1.1 \AA$ FWHM of the filter employed by SUFI, which includes not just the $\mathrm{H}_{2}$ and $\mathrm{H}_{3}$ features of the line core, but also parts of the inner wings. The $\mathrm{H}_{2}$ and $\mathrm{H}_{3}$ features sample the lower to middle chromosphere, while the inner wings are mainly sensitive to the upper photosphere (see the heights of formation computed by Danilovic et al. 2014; Jafarzadeh et al. 2017).

The results of the present analysis allow us to relate SCFs to small-scale elongated, chromospheric structures already known from the literature. In Table 2.2 we list the properties of the SCFs together with those of type I and type II spicules (from Pereira et al. 2012), and of Ca II K fibrils (as deduce by Pietarila et al. 2009). Spicules show considerable similarities to the SCFs with regard to the range of widths (although the Ca fibrils studied by us and, in particular, those by Pietarila et al. are on average narrower).

The difference in width between the fibrils seen by Pietarila et al. in Ca II K and those studied by us in $\mathrm{Ca} I \mathrm{H}$ is puzzling. It has at least partly to do with the difference in technique, as the application of our technique to a sample of fibrils identified by Pietarila et al. (2009) returns larger widths than those found by those authors. Other factors may also contribute to the difference in width. Thus, it may have to do with different optical thicknesses, or that our observations were recorded in a larger, more intensely active region.

Both types of known spicules are considerably longer than our SCFs, with even the 
Table 2.2: Comparison of some morphological properties between SCFs observed in $\mathrm{Ca}$ II $\mathrm{H}$ and in and $\mathrm{Ca}$ II $\mathrm{K}$ spicules.

\begin{tabular}{rlcc}
\hline \hline & Lifetime & Width & Length \\
\hline SCFs (this work) & $35-450 \mathrm{~s}$ & $100-500 \mathrm{~km}$ & $0.5-4.5 \mathrm{Mm}$ \\
& $(35-2000 \mathrm{~s})^{a}$ & & \\
mean value & $80 \mathrm{~s}$ & $182 \mathrm{~km}$ & $1.38 \mathrm{Mm}$ \\
\hline Type I spicules $^{2}$ & $150-400 \mathrm{~s}$ & $100-700 \mathrm{~km}$ & $4-8 \mathrm{Mm}$ \\
mean value & $262 \mathrm{~s}$ & $348 \mathrm{~km}$ & $6.87 \mathrm{Mm}$ \\
\hline Type II spicules $^{b}$ & $50-150 \mathrm{~s}$ & $100-700 \mathrm{~km}$ & $3-9 \mathrm{Mm}$ \\
mean value & $165 \mathrm{~s}$ & $319 \mathrm{~km}$ & $7.75 \mathrm{Mm}$ \\
\hline Ca II K $^{c}$ & & $75-150 \mathrm{~km}$ & \\
mean value & & $100 \mathrm{~km}$ & $\approx 0.9 \mathrm{Mm}$ \\
\hline
\end{tabular}

${ }^{a}$ after taking into account the intermittent fibrils, see Sect. 2.5.1
${ }^{b}$ Pereira et al. (2012)
${ }^{c}$ Pietarila et al. (2009)

shortest spicules being close to the longest SCFs that we find. This difference in length may be a selection effect, however, as only the longer spicules are clearly identified at the limb, whereas shorter ones are buried in the forest of features at the solar limb. Conversely, our technique most likely underestimates the lengths of the fibrils, as it often fails to follow the fibrils to their usually faint ends and often identifies the undisturbed fragments of a fibril (lying, e.g., between locations at which the fibril crosses other structures) as complete, separate fibrils. The lifetimes of spicules appear to lie in between those we obtain without considering SCFs to survive gaps and those obtained after interpolating across such gaps.

Such a similarity in some properties does not imply that SCFs and spicules both occur in similar magnetic structures. Firstly, spicules are clearly identified only at some distance from the limb, i.e., at a much greater height than the SCFs, which are clearly located in the low chromosphere. Secondly, whereas spicules are more or less outward directed, as clearly shown by limb observations, the SCFs we see are well visible on the disk over a distance of up to $10^{4} \mathrm{~km}$, which implies that they are nearly horizontal. This conclusion is supported by the comparison carried out by Jafarzadeh et al. (2017) between the SCFs imaged by SUFI and the magnetic field extrapolated into the chromosphere on the basis of a magnetostatic equilibrium (Wiegelmann et al. 2017). Jafarzadeh et al. (2017) found that the azimuthal directions of both agreed very well, that the magnetic field lines corresponding most closely to the fibrils remained nearly horizontal. These field lines also mostly returned to the solar surface, thus forming very flat, low-lying magnetic loops. However, there are also (shorter) fibrils that are associated with field lines that reach up into the corona. These may be more closely related to spicules.

Also, we find that brightenings in around 50\% of the SCFs start in the body of the fibril, rather than at its edge. These features are obviously not driven from one footpoint, 
as are jet-like structures such as spicules. For another $\approx 25 \%$ of the SCFs, the brightness variation is similar to that presented in Figure 2.11, with the two edge-points of the SCF being brighter than the central part, which is also not compatible with a jet-like structure, where only one end of the structure is rooted in the photosphere, from where it points toward the upper layers of the atmosphere. All this suggests that our SCFs are more likely to outline small, loop-like structures, an interpretation which is also supported by the analysis of the curvature computed in the Sect. 2.5.4. The brightness enhancement at both ends of the SCFs may be a result of a footpoint heating process, although the structure may simply reflect the fact that the broad Ca filter of SUFI allows the photospheric bright points to shine through. This is very similar to the structures seen by Pietarila et al. (2009), who also found many of their narrow fibrils to be rooted in underlying bright points.

The radiation transmitted through the SUFI $1.1 \AA \mathrm{Ca}$ II $\mathrm{H}$ filter comes from either the photosphere, or the lower chromosphere (average height of $600-700 \mathrm{~km}$ ). This is very close to the limit of the small-scale canopies created by the granulation (WedemeyerBöhm et al. 2008), which are also found by Jafarzadeh et al. (2017) on the basis of the magnetohydrodynamic-simulation assisted Stokes inversion output of Riethmüller et al. (2017). Some of the shorter SCFs may indeed be associated with the small-scale granular canopies, with possible similarities to the seething horizontal magnetic fields described in Harvey et al. (2007), or the horizontal fields detected by Hinode (Lites et al. 2008) and SUNRISE (Danilovic et al. 2010). However, the longer SCFs are likely more closely related the larger-scale canopy found over the network by Jones and Giovanelli (1982) and modeled by Solanki and Steiner (1990). Such extended horizontal field lines were found lying nearly parallel to the SCFs in the magnetostatic equilibrium computed by Wiegelmann et al. (2017). 



\section{Paper II- Oscillations on width and intensity of slender Ca II H fibrils ${ }^{1}$}

\subsection{Abstract}

We report the detection of oscillations in slender Ca II H fibrils (SCFs) from high-resolution observations acquired with the SUNRISE balloon-borne solar observatory. The SCFs show obvious oscillations in their intensity, but also their width. The oscillatory behaviors are investigated at several positions along the axes of the SCFs. A large majority of fibrils show signs of oscillations in intensity. Their periods and phase speeds are analyzed using a wavelet analysis. The width and intensity perturbations have overlapping distributions of the wave period.

The obtained distributions have median values of the period of $32 \pm 17 \mathrm{~s}$ and $36 \pm$ $25 \mathrm{~s}$, respectively. We find that the fluctuations of both parameters propagate in the SCFs with speeds of $11_{-11}^{+49} \mathrm{~km} \mathrm{~s}^{-1}$ and $15_{-15}^{+34} \mathrm{~km} \mathrm{~s}^{-1}$, respectively. Furthermore, the width and intensity oscillations have a strong tendency to be either in anti phase or, to a smaller extent, in phase. This suggests that the oscillations of both parameters are caused by the same wave mode and that the waves are likely propagating. Taking all the evidence together, the most likely wave mode to explain all measurements and criteria is the fast sausage mode.

\subsection{Introduction}

Magnetohydrodynamic (MHD) waves have been observed in various plasma structures in the solar atmosphere, particularly in elongated features in the solar chromosphere and in the corona (for recent reviews see, e.g., Banerjee et al. 2007; Zaqarashvili and Erdélyi 2009; Mathioudakis et al. 2013; Jess et al. 2015). According to the theory of MHD oscillations, the waves may appear as a single mode or as a combination of several modes (i.e., kink, sausage, torsional, or longitudinal) with distinct properties and different observational signatures (Edwin and Roberts 1983). These waves are often excited at photospheric heights (e.g. by granular buffeting, Evans and Roberts 1990, or vortex motion, Kitiashvili et al. 2011), and either propagate away from their source or form a standing oscillation.

\footnotetext{
${ }^{1}$ This chapter reproduces the article Morphological properties of Ca II H slender fibrils by R. Gafeira,S. Jafarzadeh,S. K. Solanki, A. Lagg, M. van Noort, P. Barthol, J. Blanco Rodríguez, J. C. del Toro Iniesta, A. Gandorfer, L. Gizon, J. Hirzberger, M. Knölker, D. Orozco Suárez, T. L. Riethmüller, and W. Schmidt, published in ApJS, 229, 2017, DOI 10.3847/1538-4365/229/1/7. Reproduced with permission of ApJS
} 
While transverse waves such as kink or global Alfvénic modes cause the swaying of a flux tube or of an elongated feature, sausage-mode oscillations result in a periodic axisymmetric expansion and contraction of the structure at one position. Torsional or twisting motions are associated with torsional Alfvén waves propagating along the axis of fibrillar structures (e.g., Spruit 1982; Solanki 1993a).

The different wave modes in elongated structures have been mostly observed at coronal heights in, e.g., coronal loops (e.g., Aschwanden et al. 1999; De Moortel et al. 2002; Wang et al. 2002; Wang and Solanki 2004; Tomczyk et al. 2007; Srivastava et al. 2008; Nakariakov et al. 2012, see Nakariakov and Verwichte 2005 for a review), as well as at the upper chromospheric levels in features such as filaments, fibrils, mottles, and spicules (De Pontieu et al. 2007; Lin et al. 2007; Okamoto and De Pontieu 2011; Pietarila et al. 2011; Tsiropoula et al. 2012).

Images recorded in the Ca II K passband (with a filter width of $1.5 \AA$ ) with the $1 \mathrm{~m}$ Swedish Solar Telescope (Scharmer et al. 2003b) in an active region close to the solar disk center revealed the presence of slender, bright fibrils, extending seemingly horizontally in the lower chromosphere (Pietarila et al. 2009). Only recently, the second flight of the 1-m balloon-borne solar observatory SUNRISE (Solanki et al. 2010; Barthol et al. 2011; Berkefeld et al. 2011; Solanki et al. 2017) provided us with a high quality, seeing-free time-series of Ca II H images (with a filter width of $1.1 \AA$ ). The relatively long duration of the observations (one hour) in an active region (close to disk center) enabled a thorough study of properties of the slender fibrils (Gafeira et al. 2017b). In addition, Jafarzadeh et al. (2017) have found ubiquitous transverse waves in the slender Ca II H fibrils (SCFs) in SUNRISE data. The SCFs have been shown to map the magnetic fields in the low solar chromosphere (Jafarzadeh et al. 2017). Earlier, (Pietarila et al. 2009) had shown that the fibrils, or the magnetic canopy outlined by them, either suppressed oscillations or channeled low frequency oscillations into the chromosphere, depending on their location.

In this paper we investigate width and intensity oscillations in the SCFs observed with SUNRISE. Periods of the fluctuations in individual SCFs are determined and the phase speed of the waves propagating along the thin structures are quantified (Sect. 3.4). We discuss our results in Sect. 3.5, where we also conclude that the observed oscillations are likely manifestations of sausage waves traveling along the fibrils.

\subsection{Observations}

For the present study we use the data set described in Gafeira et al. (2017b), Jafarzadeh et al. (2017), and Solanki et al. (2017). The data set includes high spatial and temporal resolution observations of an active region obtained in the $\mathrm{Ca}$ II $\mathrm{H}$ passband (with a full width at half maximum, FWHM, of $\approx 1.1 \AA$ ) of the SUNRISE Filter Imager (SUFI, Gandorfer et al. 2011) on the 1-meter SUNRISE balloon-borne solar observatory (Solanki et al. 2010; Barthol et al. 2011; Berkefeld et al. 2011) during its second science flight (Solanki et al. 2017). The observations were obtained between 23:39 UT on 2013 June 12 and 00:38 UT on 2013 June 13 with a cadence of $7 \mathrm{~s}$. These observations covered a part of NOAA AR 11768, mainly its following polarity that was dominated by a series of pores, some of which lie at least partly within the SUFI field-of-view (FOV). The FOV also contained a number of plage elements and two granulation-scale flux emergences 
(e.g., Centeno et al. 2017). The FOV was centered at $\mu=\cos \theta=0.93$, where $\theta$ is the heliocentric angle.

Figure 3.1 illustrates a $\mathrm{Ca} I \mathrm{H}$ image (right panel) along with its co-spatial and cotemporal photospheric filtergram (left panel) recorded at $300 \mathrm{~nm}$ with the SUNRISE/SUFI instrument. The arrow on the Ca II H image marks an example SCF studied here. All the intensity values where normalized to the mean intensity of the relatively quiet region, indicated by the white box in the top left corner of the Figure 3.1.

The Ca II H image has both, a photospheric and a low chromospheric component. The latter is strongly enhanced in an active region (Danilovic et al. 2014; Jafarzadeh et al. 2017). The fibrils dominating much of the Ca II H image are expected to be located in the lower chromosphere, as none of the photospheric channels on SUNRISE even remotely shows any signs of such fibrils (see Jafarzadeh et al. 2017).

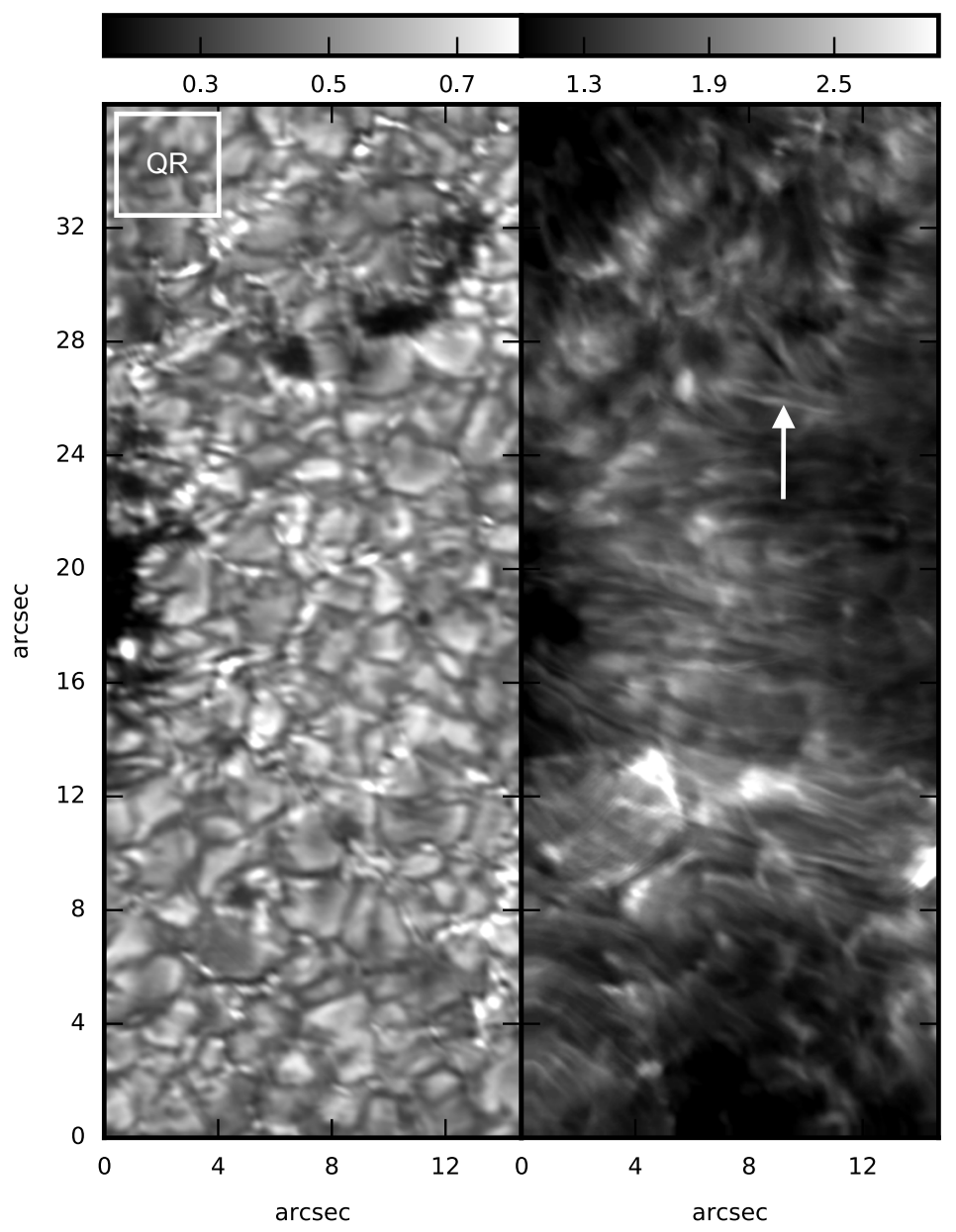

Figure 3.1: Right: Example of a Ca II H image recorded by SUNRISE/SUFI. Left: An image recorded at $300 \mathrm{~nm}$, aligned with the Ca II H filtergram. The white arrow in the right panel indicates a sample slender $\mathrm{Ca}$ II $\mathrm{H}$ fibril (SCF). The white box marks the quiet region $(\mathrm{QR})$ used to normalize the intensities in the $\mathrm{Ca}$ II $\mathrm{H}$ and $300 \mathrm{~nm}$ images. 


\subsection{Analysis and results}

To analyze both the intensity and width variations of the SCFs with time, we must first extract the fibrils from the $\mathrm{Ca} I \mathrm{H}$ filtergrams. We follow the identification and tracking method described in detail in Gafeira et al. (2017b). First, this method defines a binary mask of the fibrils. This mask is obtained by applying an unsharp masking and an adaptive histogram equalization method to the intensity images to increase their contrast. In these images, a threshold of $50 \%$ of the maximum intensity defines the binary mask isolating the fibrils from the background. All features smaller than the diffraction limit of the telescope are discarded. For the temporal evolution of a fibril we require at least 10 pixels of the fibril to be visible at the same position in at least 5 out of 6 subsequent frames, corresponding to a minimum lifetime of $35 \mathrm{~s}$. In all of frames, a fibril backbone is defined as the line equidistant to the fibril's border. A second-order polynomial is fitted to all backbones of the individual fibrils. Fibrils of complex shape that are poorly fitted are excluded from the analysis. We extend this fitted curve by $0^{\prime \prime} .3$ in both end points, the approximate width of a fibril, to compensate for the reduction of the fibril to a single-pixel structure. The resulting line is what we call the reference backbone (see the red line in Figure 3.2). For more details, we refer to Gafeira et al. (2017b). This reference backbone is the central line for the mesh, displayed in Figure 3.2. All other points of the mesh are calculated from equally spaced lines perpendicular to this reference backbone.

This mesh is based on the fibril backbone, i.e. the temporal average of all fibrils, and is therefore time independent, allowing the study of the temporal variations of its brightness and width. We use a mesh with a fixed total width of $1^{\prime \prime} .2$ for all the fibrils, while the length is the same as the reference backbone of each individual SCF. This procedure results in a total number of 598 detected SCF over the full data set with lifetimes of $35 \mathrm{~s}$ or longer.

Using this approach, we straightened each identified SCF in each image (observed at different times) by interpolating its intensity onto every point of the mesh. After these steps we can represent the SCF along a straight line, as shown in Figure 3.3 for an example fibril pictured at 40 timesteps. Every black-framed box in Figure 3.3 includes the straightened SCF at a given time. This way of stacking the temporal snapshots of a fibril allows us to easily follow fluctuations of its intensity (and with some additional effort also of its width) at any location along the reference backbone of the SCF during its entire lifetime. Thus we can identify different types of oscillations/pulsations in these structures. The example shown in Figure 3.3 illustrates a clear fluctuation of the intensity with a period of approximately $147 \mathrm{~s} \mathrm{(21} \mathrm{frames),} \mathrm{indicated} \mathrm{by} \mathrm{the} \mathrm{double-headed} \mathrm{vertical} \mathrm{arrow.}$

To inspect the fluctuations in both the intensity relative to the mean intensity of the quiet Sun and the width of the SCFs in detail, we evaluate the intensity at 17 positions along the backbone of the fibril (lying between $20 \%$ and $80 \%$ of the full length of the reference backbone measured from one of its ends), perpendicular to which we create artificial slits (that correspond to a given set of $x$ positions in the mesh frame). We then compute the position of the maximum intensity of the fibril, the intensity at this position, as well as the width of the fibril along each of these slits following the method outlined further below.

In some cases, a second fibril may be present inside the mesh determined for one fibril, usually near the edge of the mesh. As a result, more than one local maximum is present 


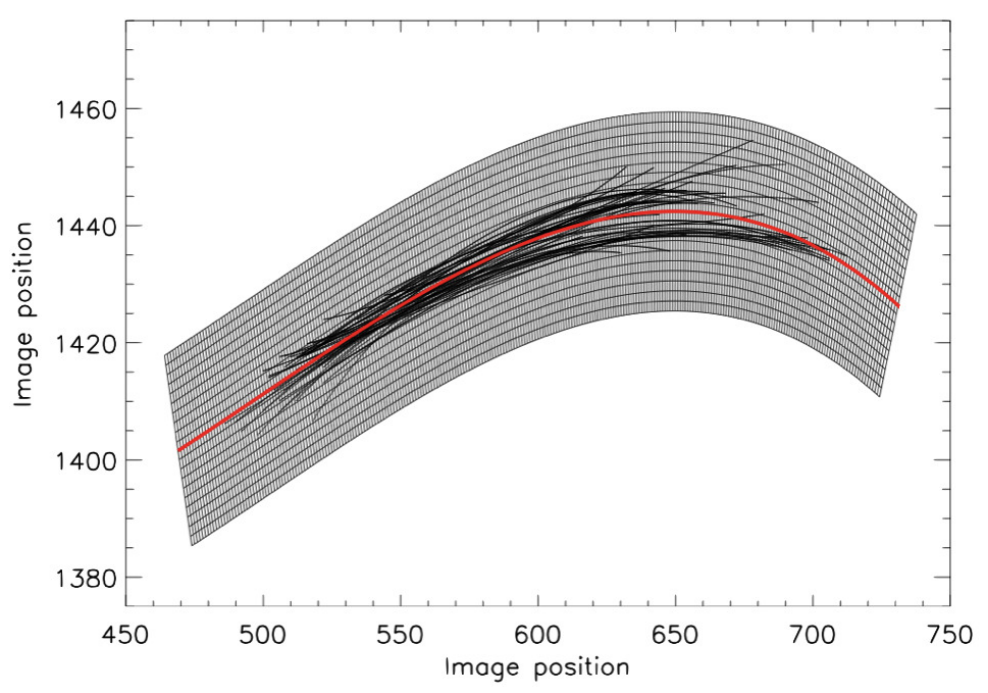

Figure 3.2: Illustration of backbones of a sample SCF (at different times and averaged) and the grid associated with the SCF. The individual backbones of this fibril determined in the images recorded at different times are represented by the individual black lines. The reference backbone is represented by the red line and the mesh is shown by the black grid.

along the artificial slit used for the determination of the fibril's width. In such cases, we choose the local maximum that is closer to the center of the mesh, i.e., the reference backbone of the fibril.

The width of a fibril is computed by fitting a Gaussian function plus a linear background to the intensity profile perpendicular to the backbone. To minimize the influence of neighboring fibrils, the six points closest to the maximum intensity position are given $30 \%$ higher weights. The FWHM of the fitted Gaussian defines the fibril width. In Figure 3.4 we present an example of these measurements, where positions of the maximum intensity (red circles) and the width of the fibril (vertical black lines) are marked at various positions along the SCF (within each image) and at different times (from one image in the stack to the next). For better visibility, we have chosen a relatively short-lived SCF for clarity (i.e., we need to show fewer timesteps). The plot clearly shows that the width is bigger in the brighter part of the fibril. The way the width is determined (see above) is independent of the fibril's intensity as long as the profile shape of the intensity perpendicular to the fibril's axis does not change and the fibril's intensity is higher than the background. We note that the locations along the fibrils are determined only as long as the intensity along the SCF is larger than the average intensity of the image at each time step. Therefore, the positions close to the left end of the example SCF shown in Figure 3.4 are not detected at all times.

\subsubsection{Wavelet analysis}

We apply a wavelet analysis to characterize the temporal variation of the power spectrum of the width and intensity oscillations. We use the wavelet algorithm described by Jafarzadeh et al. (2017). For the cases with a clear intensity and width oscillation, we also 


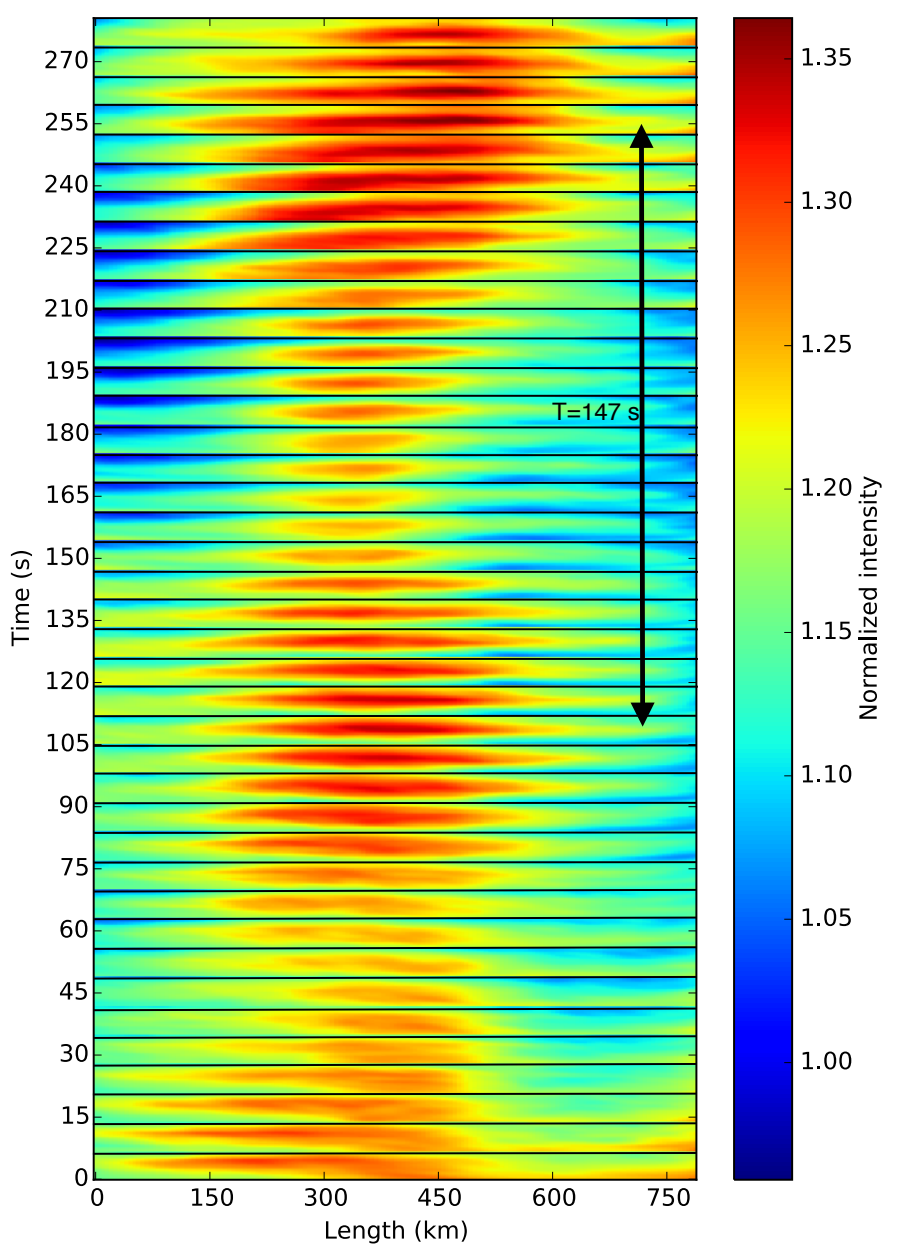

Figure 3.3: Temporal variation of a SCF. The images of a straightened fibril at different times are vertically stacked. Individual images, recorded every $7 \mathrm{~s}$, are separated by horizontal black lines. The vertical arrow indicates the period of the fibril's intensity fluctuation. The colour represents intensity, normalized to the mean value of the quiet region marked in Figure 3.1.

calculate cross-power spectra, i.e., the multiplication of the wavelet power spectrum of the oscillation in a given quantity at one position along the fibril by the complex conjugate of the same wavelet power spectrum at a different location along the same fibril. This provides us with the phase differences between the consecutive positions in width and intensity oscillations, and hence, with the phase speed of the waves along the fibrils. Finally, we also determine the wavelet cross-power spectrum between brightness and width oscillations that provides the phase difference between them.

In some cases, the determination of the maximum intensity and width of a fibril at some positions along the fibril at a given time is difficult, leading to gaps in some of the 17 positions along the fibril backbone. These gaps are filled by linearly interpolating in time to provide the wavelet analysis with equidistant data points. Such interpolations can 


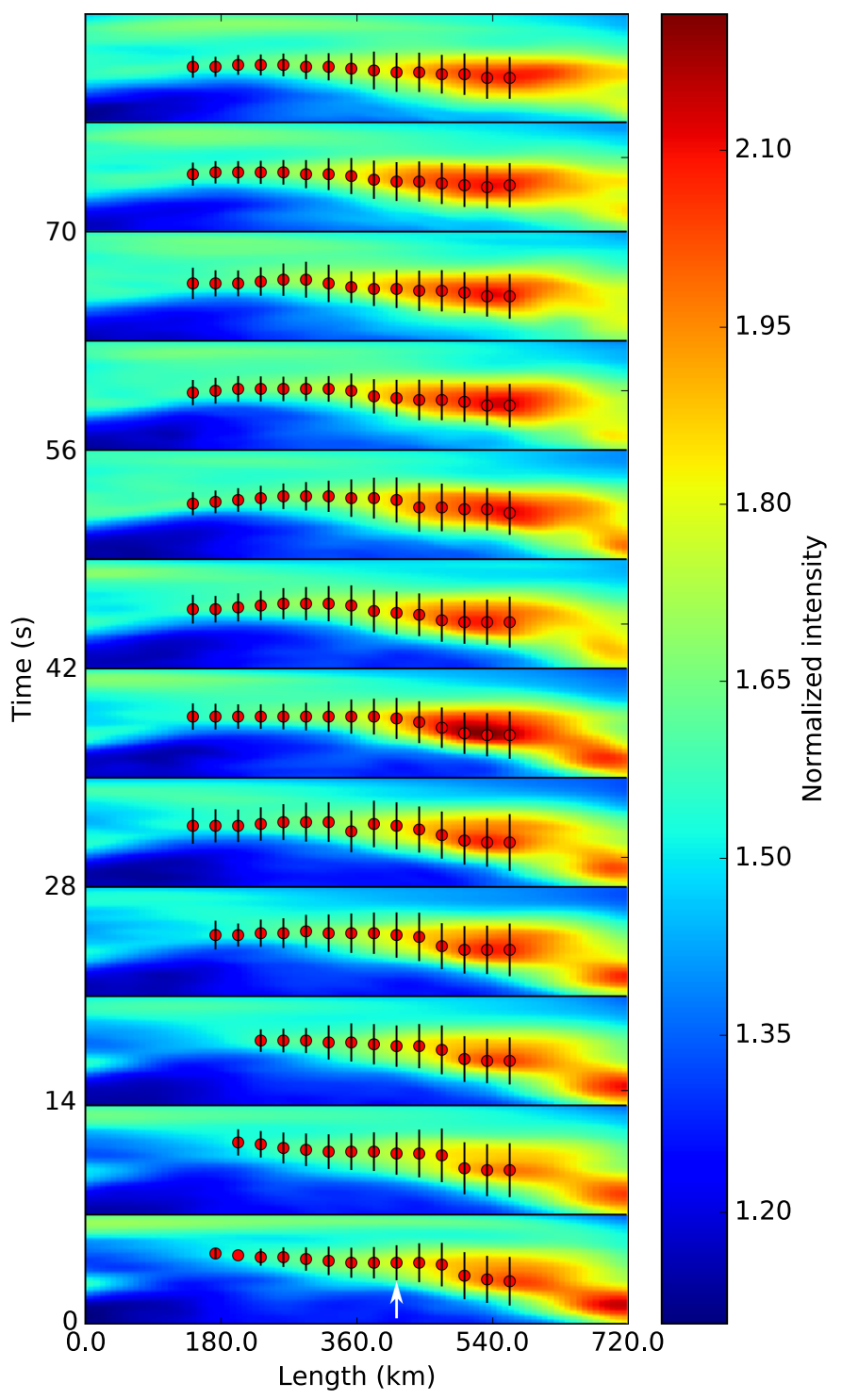

Figure 3.4: An example of intensity maxima and width detections along cuts perpendicular to the axis of a SCF. Plotted are vertically stacked images of a fibril recorded in Ca II $\mathrm{H}$ observed at different times. Individual images, recorded every $7 \mathrm{~s}$, are separated by horizontal black lines. The red dots within a given image represent the locations of the fibril's maximum intensity along a series of cuts roughly perpendicular to the backbone of the fibril, while the vertical black lines indicate the width of the fibril at the same locations. The colour represents intensity, normalized to the mean value of the quiet region in the SUFI frame (marked in Figure 3.1). The white arrow in the lower part of the bottom image marks the location at which the oscillations plotted in Figure 3.7 occurred. Note that this fibril is not the same as presented in Figure 3.3.

result in overestimation of, e.g., periods of the oscillations. Gaps are sufficiently rare, however, that their influence turned out to be relatively insignificant. We find that $74 \%$ of 
the fibrils display above $95 \%$ confidence level (inside the cone of influence) oscillations in intensity, with on average $42 \%$ of the cuts along each oscillating fibril displaying such an oscillation. Similarly, $82 \%$ of the fibrils exhibit oscillations of the width, whereby $38 \%$ of the cuts through the backbone of oscillating fibrils show the oscillations (on average). For the fibrils displaying an oscillation with a sufficiently high confidence, the frequency at which the wavelet power spectrum has its strongest peak is taken as the period of the oscillation (within a given fibril). The most likely phase speed of the wave is determined in the same way from the wavelet cross-power spectrum between different spatial locations along a fibril. Only the highest peaks that are above the 95\% confidence level and inside the cone of influence (i.e., frequency-time areas that are not influenced by the ends of the time series) are considered.

\subsubsection{Statistics}

The two-dimensional histogram of intensity and width periods presented in Figure 3.5 demonstrates that most of the fibrils oscillate with periods between 20 and $40 \mathrm{~s}$ in both quantities, with median values of $32 \pm 17 \mathrm{~s}$ and $36 \pm 25 \mathrm{~s}$ for the periods of the width and intensity oscillations, respectively. For a large fraction of the fibrils $(\approx 75 \%)$ the periods in both quantities are similar. For the phase speeds, we obtain median values of $11_{-11}^{+49} \mathrm{~km} \mathrm{~s}^{-1}$ and $15_{-15}^{+34} \mathrm{~km} \mathrm{~s}^{-1}$ for width and intensity oscillations, respectively, without any correlation between them. These median periods are rather short, well below the cutoff frequency of the atmosphere, while the phase speeds are above the sound speed in the temperature minimum region and lower chromosphere.

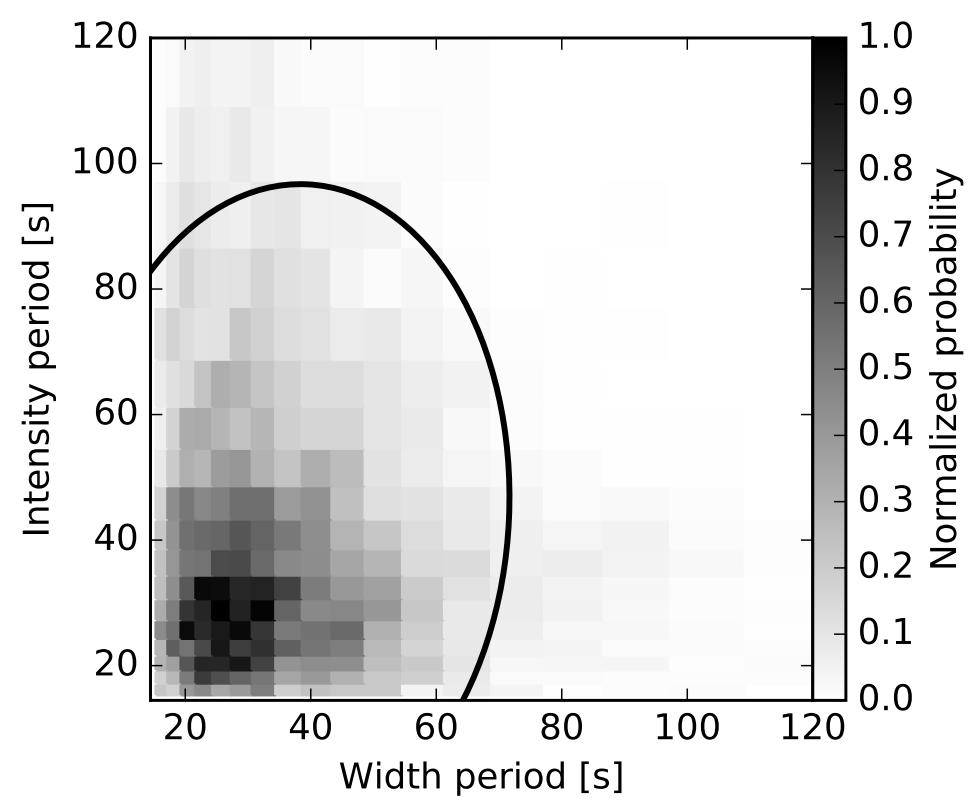

Figure 3.5: Two-dimensional histogram showing the relation between the periods of the width and the intensity oscillations in SCFs. The bin size follows the period resolution that is limited by the lifetime of the fibrils. The black curved line indicates the $95 \%$ confidence level. 
We computed the wavelet cross-power spectra between width and intensity oscillations within a given cut across an SCF and determined the phase lag between the oscillations in these quantities. A wide range of phase lags was obtained. Figure 3.6 illustrates the distribution of values of this quantity. The distribution has two clear peaks, a weaker one at $0^{\circ}$ (in phase) and strong peak at $\pm 180^{\circ}$ (anti phase). As an example for such an anti-phase oscillation, we present in Figure 3.7 the temporal evolution of fibril width and intensity of the sample SCF at the position indicated by a white arrow in Figure 3.4.

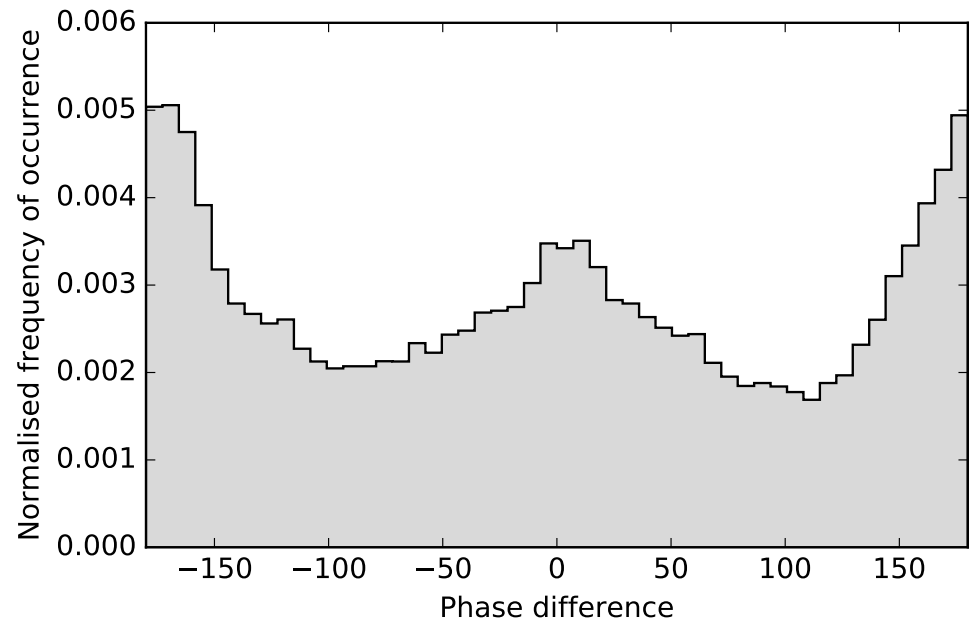

Figure 3.6: Distribution of phase differences between width and intensity oscillations in the SCFs at a given cut across each fibril.

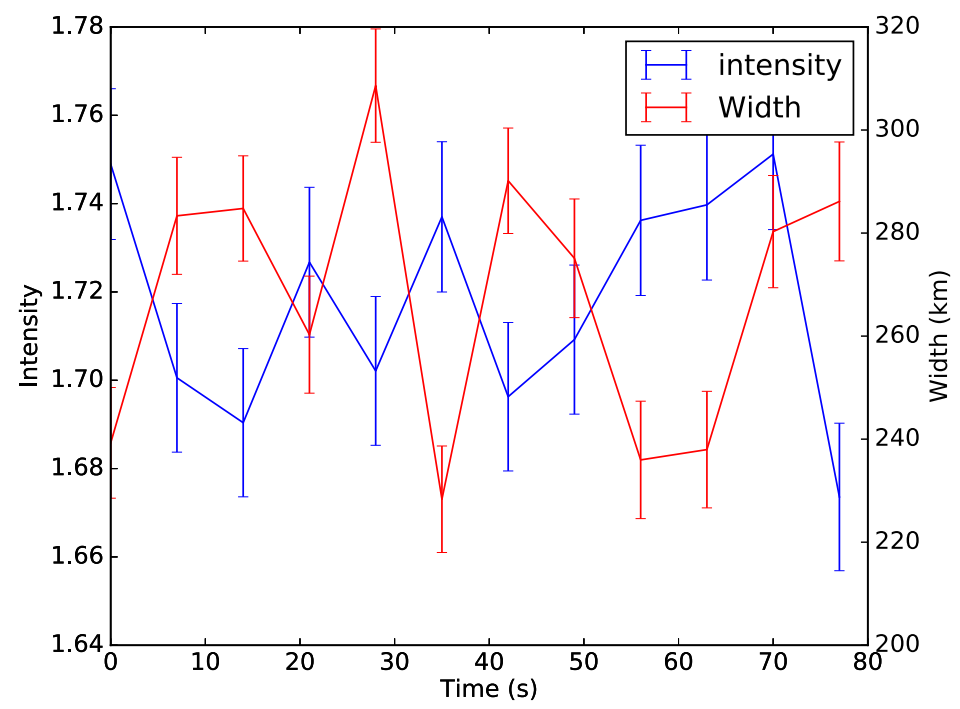

Figure 3.7: Example of a clear anti-correlation between oscillations in maximum intensity (blue line) and width (red line) of the sample SCF in Figure 3.4, at the location marked by the white arrow in the lowest panel of that figure. The error bars represent the standard deviations of the photon counts for the intensity and the uncertainties of the Gaussian fitting to the cross-section of the fibril for the width. 


\subsection{Discussion and conclusions}

We have provided observational evidence for oscillations of the width of fibrils and of intensity along SCFs in the lower chromosphere.

The high-resolution seeing-free images under study were recorded with SUNRISE/ SUFI and revealed that such oscillations are almost ubiquitous all over the field of view, which covered part of NOAA AR 11768. Fluctuations in length of some of the SCFs were also observed, although it is not clear how independent this parameter is from the intensity.

The oscillatory behavior of both parameters (i.e., fibril width and intensity) was identified in wavelet power spectra determined at a series of locations along the backbone of each detected SCF. The wavelet transform was employed to analyze the fluctuations from which median periods of $32 \pm 17 \mathrm{~s}$ and $36 \pm 25 \mathrm{~s}$ were obtained for the width and intensity oscillations, respectively, with the uncertainty intervals representing the standard deviations of each distribution. Cross-power spectra between the perturbations at different locations along a given fibril revealed phase speeds of $11_{-11}^{+49} \mathrm{~km} \mathrm{~s}^{-1}$ and $15_{-15}^{+34} \mathrm{~km} \mathrm{~s}^{-1}$ for the width and intensity fluctuations, respectively. Again, uncertainty intervals reflect standard deviations. Given that these waves display brightness and width signatures, they have to be compressible, ruling out Alfvén waves. Simultaneous periodic fluctuations of intensity and width in elongated structures are manifested by either slow-mode waves or fast sausage-mode waves (Van Doorsselaere et al. 2011; Su et al. 2012). In addition to observations of the latter wave mode in the upper solar atmosphere (i.e., in the upper chromosphere and in the corona, Inglis et al. 2009), observations of sausage oscillations have also been reported at lower atmospheric heights, in structures such as pores (Dorotovič et al. 2008; Morton et al. 2011).

To distinguish which wave modes are present in the SCFs we need to compare the phase speeds of the oscillations with the expected plasma Alfvén and sound speeds. The values for the Alfvén and sound speeds were computed using the NC5 flux tube from Bruls and Solanki (1993) embedded in the VAL-A atmosphere (Vernazza et al. 1981). For a majority of the detected waves, these velocities are larger than the sound speed at the low chromospheric heights sampled by the SUFI $1.1 \AA \mathrm{Ca} I \mathrm{H}$ filter, which lies around $7 \mathrm{~km} \mathrm{~s}^{-1}$. The measured phase speeds are comparable to the local Alfvén speed for this height region, with typical values in the range of $7-25 \mathrm{~km} \mathrm{~s}^{-1}$.

Interestingly, $20 \%$ of the SCFs show phase differences between $-30^{\circ}$ and $+30^{\circ}$, indicative of in-phase oscillations.

A strong peak was found at $\pm 180^{\circ}$, with about $50 \%$ of all oscillations having a phase difference within $30^{\circ}$ of $180^{\circ}$ (anti-phase oscillations). Phase differences of around $\pm 90^{\circ}$ are relatively uncommon.

A phase difference in the range of $\left|150^{\circ}-180^{\circ}\right|$, as displayed by the example shown in Figure 3.7, is consistent with the signature of sausage-mode oscillations in the SCFs under the assumption of an optically thin plasma. The validity of this assumption is confirmed by the fact that the observed intensity increases at the intersection points of crossing SCFs, suggesting that we can partly see through individual fibrils. The contraction of the fibril caused by the sausage-mode oscillation leads to a narrow fibril with a higher density. In an optically thin regime, a higher density implies an increased intensity. The subsequent expansion phase of the oscillation leads to an increase of the fibril's width a 
lower intensity. For such a plasma, the intensity follows the electron density. However, a detailed model of the brightening of these structures is required to determine the behavior of the plasma under conditions typical of the lower chromosphere.

In about $25 \%$ of our SCFs we did not find a clear correlation between the fluctuations in the two parameters. The intensity oscillations could also be caused by slow-mode waves, which are expected to be present inside strong-field magnetic features, such as flux tubes (although it is unclear to what extent the SCFs can be described as flux tubes). However, the median phase speeds obtained in our analysis are too high for slow-mode waves. Only for a few SCFs do we obtain low phase speeds that may well be compatible with the slow mode.

To our knowledge, our observations of sausage-mode oscillations in the SCFs are the first direct evidence of this wave mode in the lower solar chromosphere. Morton et al. (2012) inspected oscillations of width and intensity in $\mathrm{H} \alpha$ elongated fibrils and short mottles (in the upper chromosphere). They found a phase speed of $67 \pm 15 \mathrm{~km} \mathrm{~s}^{-1}$ for their MHD fast sausage waves, which is much larger than those we found in the SCFs. Like us, they also found a phase difference of $180^{\circ}$ between their detected intensity and width perturbations. Dorotovič et al. (2008) and Morton et al. (2012) showed that the energy these sausage waves carry is sufficient to contribute (around 10\%) to the heating of the chromosphere and/or the corona. Jess et al. (2012) claimed to see a fluctuation of the width of what they call a chromospheric spicule (observed on the disk as an $\mathrm{H} \alpha$ dark fibril). They interpret these fluctuations as sausage modes in the chromosphere.

This work points to a number of follow-up investigations to advance our knowledge and understanding of the detected oscillations and waves. Firstly, measurements that include velocities would help to distinguish better between different possible wave modes. Another important step is to compute MHD wave modes in simple models of fibrils, possibly described as flux tubes embedded in a magnetized gas. Such a study should not only lead to new insights into the physics of these oscillations, but would also reveal the expected behavior of different physical parameters, thus providing guidance for future observations and their interpretation. Finally, an investigation of the physical processes that drive this oscillatory behavior of the SCFs would also be very useful. 



\section{Conclusion and Outlook}

In this thesis, we present a detailed study of the morphological properties of slender $\mathrm{Ca}$ II H fibrils (SCFs) and their oscillatory behavior. It is composed of work presented in two publications that appeared in the Astrophysical Journal Supplement Series in 2017, as part of a SUNRISE special issue. In the first paper, reproduced in Chapter 2, we apply an automatic detection algorithm to extract all the bright elongated structures present in the $\mathrm{Ca}$ II $\mathrm{H}$ intensity maps. This algorithm is based on a series of unsharp mask procedures and contrast enhancement technique that allow us to identify in total 598 SCFs for further analysis in this thesis.

This analysis comprises of the study of the morphological properties of the SCFs and their statistics (see Table 2.1 for more details). We obtain lifetimes between $35 \mathrm{~s}$ and 30 min (taking into account the intermittency in fibril visibility), maximum lengths from 0.5 to $4.5 \mathrm{Mm}$, widths between 100 and $270 \mathrm{~km}$ and a slight preference for a positive curvature. This preferential curvature agrees with the idea of these fibrils being part of low-lying loops observed away from solar disk center.

We also compare our results with other studies of chromospheric small-scale elongated structures. In Table 2.2 we see that SCFs show some similarities with type I and type II spicules (from Pereira et al. 2012), and with Ca II K fibrils (as deduced by Pietarila et al. 2009). We emphasize that despite these similarities SCFs, spicules, and Ca II K fibrils might still outline different magnetic structures.

Two different types of the temporal evolution of the brightness of SCFs were also identified. In one type the intensity increases starting from the center and propagates to the edges, i.e., towards the footpoints of the loops outlined by the SCFs (around 50\% of the cases). In the second type the increase in intensity starts at the edges and expands from there towards the center (around 25\%). A jet-like brightness variation, as observed in spicules of both types, is not compatible with either of the two cases, since the SCFs show a symmetric brightness variation around their centers. $25 \%$ of the observed SCFs do not show a clear pattern in the brightness variation.

The second part of this thesis is dedicated to the analysis of the oscillatory behavior of SCFs. This topic is covered by the paper forming Chapter 3 and by the paper given in the appendix. In chapter 3, we focus on the oscillations in width and intensity along the SCFs. We measure their intensities and widths at several positions along their axis as shown in Figure 3.4 and use a wavelet transform to compute the wave parameters period, phase speed and phase difference between the width and the intensity oscillations. We observe that the oscillations show periods with nearly identical median values of $32 \pm 17 \mathrm{~s}$ for the width and $36 \pm 25 \mathrm{~s}$ for the intensity, which are again equal within the errors. For the phase speed we obtain values of $11_{-11}^{+49} \mathrm{~km} \mathrm{~s}^{-1}$ for the width and $15_{-15}^{+34} \mathrm{~km} \mathrm{~s}^{-1}$ for the intensity. All uncertainty intervals are standard deviations computed from the 
corresponding histograms. Due to the fact that the waves display these intensity and width oscillations, they have to be of magneto-acoustic type. We also observe in about $50 \%$ of the cases the perturbation to oscillate in anti-phase and in $20 \%$ in phase (see Figure 3.7). The facts that measured phase speeds are closer to the Alfvén speed (typical range for this height 7 to $25 \mathrm{~km} \mathrm{~s}^{-1}$ ) than to the sound speed (around $7 \mathrm{~km} \mathrm{~s}^{-1}$ ) and that the oscillations between the two properties are in anti-phase indicates that these waves are of fast sausage-mode type. For some of the examples, the phase speed is relatively low. In this case, they can be interpreted as slow sausage-mode waves, which are expected to be present in strong magnetic field flux tubes.

As described in the appendix, we also performed an analysis of the transversal waves in the SCFs (see Appendix A for more details). The transverse motions were measured by placing fixed artificial slits along the fibril axis (see Figure A.5) and then computing the transverse displacements, velocity amplitudes, periods and phase speeds of the oscillation. We obtained mean values of $33 \mathrm{~km}, 2.4 \mathrm{~km} \mathrm{~s}^{-1}, 83 \mathrm{~s}$, and $9 \mathrm{~km} \mathrm{~s}^{-1}$ respectively. In Table A. 2 we compare these wave parameters with other structures described in the literature. This showed that SCFs have, on average, smaller periods, transverse displacement, velocity amplitude and phase speeds when compared to other on-disk chromospheric structures.

The observed transverse oscillations can be interpreted as a transverse wave propagating along the fibril axis compatible with a kink-mode wave. Such a wave, with the obtained parameters, can carry energy of around $15 \mathrm{~kW} \mathrm{~m}^{-2}$ which is of the same order of magnitude as the energy needed to heat the solar chromosphere. This is also the region where we expect the energy of the wave to be dissipated, since the SCFs are mainly oriented in a horizontal direction, and do not extend to higher layers Jafarzadeh et al. (2017). However, some of the SCFs trace more vertical field lines, allowing for the transport of energy to the higher layers, possibly contributing to coronal heating. However, from our observations it is impossible to tell how much of its energy the wave is dissipating to the surroundings, if at all.

\section{Outlook}

The analysis mentioned above provides a wealth of information on the temporal evolution, the morphology and the statistical properties of the SCFs. However, there are still open questions to be answered to deepen the understanding of the SCFs:

1. How are the SCFs magnetically connected to the underlying photosphere?

2. What is the magnetic topology of the SCFs?

3. What is the relevance of the detected waves in the SCFs for transporting energy to higher layers of the solar atmosphere?

Answering these questions is important to conclude on the physical nature of these structures, e.g., if they are the possible on-disk counterparts of other chromospheric structures like spicules or Ca II K fibrils.

An important step to answer these questions is to analyze the magnetic and velocity properties of the SCFs, ideally also their height-dependence. This can be achieved 
by high-resolution, full-Stokes observations in the Ca II H\&K lines, but also by looking at other chromspheric observations, e.g., the He $10830 \AA$ triplet and combining it with measurements of the photospheric magnetic field and velocity vectors. Correlating the measurements from these two layers in the solar atmosphere will allow to clarify if the long SCFs are related to the larger-scale canopy found over the internetwork (Giovanelli and Jones 1982; Solanki and Steiner 1990; Lagg et al. 2009), if they are low-lying, smallscale magnetic loops, or if they are the on-disk counterparts of spicules. Large-aperture solar telescopes like GREGOR (Tenerife, Spain, Schmidt et al. (2012)) or the future 4m telescope Daniel K. Inouye Telescope (DKIST, Hawaii, USA, Tritschler et al. (2016)), and also the planned reflight of the SUNRISE balloon-borne observatory with significantly improved instrumentation (multi-line full-Stokes observations in the near UV and visible wavelengths) will offer the possibility for such measurements.

The measurement of the magnetic field with high temporal resolution in such structures will help to perform a more detailed analysis of the observed oscillatory behavior. These measurements will put further constraints on the type of the observed wave and therefore allow for their unambiguous identification. Modern instrumentation, like the 2-dimensional full-Stokes spectropolarimeter (MiHI) or the Fast Solar Polarimeter (FSP), developed at MPS, will be ideally suited to accomplish this task. Height-dependent inversion codes will lift the restriction of local thermodynamic equlibrium (for example NICOLE (Socas-Navarro et al. 1998) or analytically computed response function inversion codes (Milić and van Noort 2017)). Such codes are needed to fully exploit the data of the large-aperture telescopes and their attached instruments, providing the information about the conditions in the solar atmosphere from the deepest photospheric layers up to the corona needed to attack the open questions. 



\section{Bibliography}

Alissandrakis, C. E. and Macris, C. J.: 1971, Akademia Athenon Praktika 46, 107

Anan, T., Kitai, R., Kawate, T., Matsumoto, T., Ichimoto, K., Shibata, K., Hillier, A., Otsuji, K., Watanabe, H., Ueno, S., Nagata, S., Ishii, T. T., Komori, H., Nishida, K., Nakamura, T., Isobe, H., and Hagino, M.: 2010, PASJ 62, 871

Anusha, L. S., Solanki, S. K., Hirzberger, J., and Feller, A.: 2017, A\&A 598, A47

Aschwanden, M. J., de Pontieu, B., Schrijver, C. J., and Title, A. M.: 2002, Sol. Phys. 206, 99

Aschwanden, M. J., Fletcher, L., Schrijver, C. J., and Alexander, D.: 1999, ApJ 520, 880

Athay, R. G. (ed.): 1976, The solar chromosphere and corona: Quiet sun, Vol. 53 of Astrophysics and Space Science Library

Banerjee, D., Erdélyi, R., Oliver, R., and O’Shea, E.: 2007, Sol. Phys. 246, 3

Banos, G. J. and Macris, C. J.: 1970, Sol. Phys. 12, 106

Barthol, P., Gandorfer, A., Solanki, S. K., Schüssler, M., Chares, B., Curdt, W., Deutsch, W., Feller, A., Germerott, D., Grauf, B., Heerlein, K., Hirzberger, J., Kolleck, M., Meller, R., Müller, R., Riethmüller, T. L., Tomasch, G., Knölker, M., Lites, B. W., Card, G., Elmore, D., Fox, J., Lecinski, A., Nelson, P., Summers, R., Watt, A., MartínezPillet, V., Bonet, J. A., Schmidt, W., Berkefeld, T., Title, A. M., Domingo, V., GasentBlesa, J. L., del ToroIniesta, J. C., LópezJiménez, A., Álvarez-Herrero, A., Sabau-Graziati, L., Widani, C., Haberler, P., Härtel, K., Kampf, D., Levin, T., PérezGrande, I., SanzAndrés, A., and Schmidt, E.: 2011, Sol. Phys. 268(1), 1

Beck, C., Rezaei, R., and Puschmann, K. G.: 2013, A\&A 556, A127

Beckers, J. M.: 1968, Sol. Phys. 3(3), 367

Beckers, J. M.: 1972, Annual Review of Astronomy and Astrophysics 10(1), 73

Bel, N. and Leroy, B.: 1977, A\&A 55, 239

Berkefeld, T.: 2007, in Modern solar facilities-advanced solar science, p. 107 
Berkefeld, T., Schmidt, W., Soltau, D., Bell, A., Doerr, H. P., Feger, B., Friedlein, R., Gerber, K., Heidecke, F., Kentischer, T., v.d. Lühe, O., Sigwarth, M., Wälde, E., Barthol, P., Deutsch, W., Gandorfer, A., Germerott, D., Grauf, B., Meller, R., Álvarez-Herrero, A., Knölker, M., Martínez Pillet, V., Solanki, S. K., and Title, A. M.: 2011, Sol. Phys. 268(1), 103

Bogdan, T. J., Carlsson, M., Hansteen, V. H., McMurry, A., Rosenthal, C. S., Johnson, M., Petty-Powell, S., Zita, E. J., Stein, R. F., McIntosh, S. W., and Nordlund, Å.: 2003, ApJ 599, 626

Brandenburg, A., Rogachevskii, I., and Kleeorin, N.: 2016, New Journal of Physics 18(12), 125011

Brandenburg, A. and Subramanian, K.: 2004, Phys. Rep. 417(1-4), 1

Bratsolis, E., Dialetis, D., and Alissandrakis, C. E.: 1993, A\&A 274, 940

Bray, R. J. and Loughhead, R. E.: 1974, The solar chromosphere

Brown, D. S., Parnell, C. E., Deluca, E. E., Golub, L., and McMullen, R. A.: 2001, Sol. Phys. 201, 305

Bruls, J. H. M. J. and Solanki, S. K.: 1993, A\&A 273, 293

Buehler, D., Lagg, A., Solanki, S. K., and van Noort, M.: 2015, A\&A 576, A27

Carlsson, M., Hansteen, V. H., Gudiksen, B. V., Leenaarts, J., and De Pontieu, B.: 2016, A\&A 585, A4

Carlsson, M. and Stein, R. F.: 1997, ApJ 481, 500

Cauzzi, G., Reardon, K. P., Uitenbroek, H., Cavallini, F., Falchi, A., Falciani, R., Janssen, K., Rimmele, T., Vecchio, A., and Wöger, F.: 2008, A\&A 480, 515

Centeno, S., Blanco Rodríguez, J., Barthol, P., Del Toro Iniesta, J. C., and Sunrise team: 2017, ApJS 229, 2

Charbonneau, P.: 2010, Living Rev. Solar Phys 7, 3

Cheung, M. C. M., Schüssler, M., and Moreno-Insertis, F.: 2007, A\&A 461, 1163

Chitta, L. P., Peter, H., Solanki, S. K., Barthol, P., Gandorfer, A., Gizon, L., Hirzberger, J., Riethmüller, T. L., van Noort, M., Rodríguez, J. B., Iniesta, J. C. D. T., Suárez, D. O., Schmidt, W., Pillet, V. M., and Knölker, M.: 2017, ApJS 229(1), 4

Choudhuri, A. R., Auffret, H., and Priest, E. R.: 1993, Sol. Phys. 143, 49

Danilovic, S., Beeck, B., Pietarila, A., Schüssler, M., Solanki, S. K., Martínez Pillet, V., Bonet, J. A., del Toro Iniesta, J. C., Domingo, V., Barthol, P., Berkefeld, T., Gandorfer, A., Knölker, M., Schmidt, W., and Title, A. M.: 2010, ApJ 723, L149 
Danilovic, S., Hirzberger, J., Riethmüller, T. L., Solanki, S. K., Barthol, P., Berkefeld, T., Gandorfer, A., Gizon, L., Knölker, M., Schmidt, W., Blanco Rodríguez, J., and Del Toro Iniesta, J. C.: 2014, ApJ 784, 20

Danilovic, S., Solanki, S. K., Barthol, P., Gandorfer, A., Gizon, L., Hirzberger, J., Riethmüller, T. L., van Noort, M., Rodríguez, J. B., Iniesta, J. C. D. T., Suárez, D. O., Schmidt, W., Pillet, V. M., and Knölker, M.: 2017, ApJS 229(1), 5

Daubechies, I.: 1990, IEEE Trans. Inf. Theory 36, 961

de la Cruz Rodríguez, J. and Socas-Navarro, H.: 2011, A\&A 527, L8

De Moortel, I., Ireland, J., Hood, A. W., and Walsh, R. W.: 2002, A\&A 387, L13

De Pontieu, B., Erdélyi, R., and James, S. P.: 2004, Nature 430, 536

De Pontieu, B., Hansteen, V. H., Rouppe van der Voort, L., van Noort, M., and Carlsson, M.: 2007, ApJ 655, 624

de Pontieu, B., McIntosh, S., Hansteen, V. H., Carlsson, M., Schrijver, C. J., Tarbell, T. D., Title, A. M., Shine, R. A., Suematsu, Y., Tsuneta, S., Katsukawa, Y., Ichimoto, K., Shimizu, T., and Nagata, S.: 2007, PASJ 59, S655

De Pontieu, B., McIntosh, S. W., Carlsson, M., Hansteen, V. H., Tarbell, T. D., Boerner, P., Martinez-Sykora, J., Schrijver, C. J., and Title, A. M.: 2011, Science 331, 55

De Pontieu, B., McIntosh, S. W., Carlsson, M., Hansteen, V. H., Tarbell, T. D., Schrijver, C. J., Title, A. M., Shine, R. A., Tsuneta, S., Katsukawa, Y., Ichimoto, K., Suematsu, Y., Shimizu, T., and Nagata, S.: 2007, Science 318, 1574

Defouw, R. J.: 1976, ApJ 209, 266

Dorotovič, I., Erdélyi, R., and Karlovský, V.: 2008, in R. Erdélyi and C. A. Mendoza-Briceno (eds.), Waves Oscillations in the Solar Atmosphere: Heating and Magneto-Seismology, Vol. 247 of IAU Symposium, p. 351

Edwin, P. M. and Roberts, B.: 1983, Sol. Phys. 88, 179

Erdélyi, R. and Fedun, V.: 2007, Science 318, 1572

Erdélyi, R., Malins, C., Tóth, G., and de Pontieu, B.: 2007, A\&A 467, 1299

Evans, D. J. and Roberts, B.: 1990, ApJ 348, 346

Foukal, P.: 1971a, Sol. Phys. 20(2), 298

Foukal, P.: 1971b, Sol. Phys. 19(1), 59

Frazier, E. N. and Stenflo, J. O.: 1972, Sol. Phys. 27, 330 
Gafeira, R., Jafarzadeh, S., Solanki, S. K., Lagg, A., van Noort, M., Barthol, P., Berkefeld, T., Blanco Rodríguez, J., del Toro Iniesta, J. C., Gandorfer, A., Gizon, L., Hirzberger, J., Knölker, M., Martínez Pillet, V., Orozco Suárez, D., Riethmüller, T. L., and Schmidt, W.: 2017a, ApJS 229, 6

Gafeira, R., Lagg, A., Solanki, S. K., Jafarzadeh, S., van Noort, M., Barthol, P., Blanco Rodríguez, J., del Toro Iniesta, J. C., Gandorfer, A., Gizon, L., Hirzberger, J., Knölker, M., Orozco Suárez, D., Riethmüller, T. L., and Schmidt, W.: 2017b, ApJS 229, 6

Gandorfer, A., Grauf, B., Barthol, P., Riethmüller, T. L., Solanki, S. K., Chares, B., Deutsch, W., Ebert, S., Feller, A., Germerott, D., Heerlein, K., Heinrichs, J., Hirche, D., Hirzberger, J., Kolleck, M., Meller, R., Müller, R., Schäfer, R., Tomasch, G., Knölker, M., Martínez Pillet, V., Bonet, J. A., Schmidt, W., Berkefeld, T., Feger, B., Heidecke, F., Soltau, D., Tischenberg, A., Fischer, A., Title, A., Anwand, H., and Schmidt, E.: 2011, Sol. Phys. 268, 35

Gary, G. A.: 2001, Sol. Phys. 203, 71

Giovanelli, R. G. and Jones, H. P.: 1982, Sol. Phys. 79, 267

Gold, T.: 1964, NASA Special Publication 50, 389

Gonsalves, R. A. and Chidlaw, R.: 1979, in A. G. Tescher (ed.), Applications of Digital Image Processing III, Vol. 207 of SPIE Conf. Ser., p. 32

Goossens, M., Van Doorsselaere, T., Soler, R., and Verth, G.: 2013, ApJ 768, 191

Graham, J. P., Danilovic, S., and SchÃijssler, M.: 2009, ApJ 693(2), 1728

Grossmann-Doerth, U. and Schmidt, W.: 1992, A\&A 264, 236

Hale, G. E.: 1908, ApJ 28, 315

Hansteen, V. H., De Pontieu, B., Rouppe van der Voort, L., van Noort, M., and Carlsson, M.: 2006, ApJ 647, L73

Harvey, J. W., Branston, D., Henney, C. J., Keller, C. U., SOLIS, and Teams, G.: 2007, ApJLetters 659(2), L177

Hasan, S. S. and van Ballegooijen, A. A.: 2008, ApJ 680, 1542

Hathaway, D. H., Wilson, R. M., and Reichmann, E. J.: 1994, Sol. Phys. 151, 177

He, J., Marsch, E., Tu, C., and Tian, H.: 2009, ApJ 705, L217

Henriques, V. M. J. and Kiselman, D.: 2013, A\&A 557, A5

Henriques, V. M. J., Scullion, E., Mathioudakis, M., Kiselman, D., Gallagher, P. T., and Keenan, F. P.: 2015, A\&A 574, A131

Hirzberger, J.: 2003, Astronomische Nachrichten 324, 344 
Hirzberger, J., Feller, A., Riethmüller, T. L., Gandorfer, A., and Solanki, S. K.: 2011, A\&A 529, A132

Hirzberger, J., Feller, A., Riethmüller, T. L., Schüssler, M., Borrero, J. M., Afram, N., Unruh, Y. C., Berdyugina, S. V., Gandorfer, A., Solanki, S. K., Barthol, P., Bonet, J. A., Martínez Pillet, V., Berkefeld, T., Knölker, M., Schmidt, W., and Title, A. M.: 2010, ApJ 723, L154

Howard, R. and Stenflo, J. O.: 1972, Sol. Phys. 22, 402

Inglis, A. R., Van Doorsselaere, T., Brady, C. S., and Nakariakov, V. M.: 2009, A\&A $\mathbf{5 0 3}(2), 569$

Jafarzadeh, S.: 2013, Ph.D. thesis, Georg-August-Universität Göttingen

Jafarzadeh, S., Rutten, R., Solanki, S. K., Wiegelmann, T., Riethmüller, T. L., van Noort, M., Szydlarsky, M., Blanco Rodríguez, J., Barthol, P., del Toro Iniesta, J. C., Gandorfer, A., Gizon, L., Hirzberger, J., Knölker, M., Martínez Pillet, V., Orozco Suárez, D., and Schmidt, W.: 2017, ApJS 229, 10

Jafarzadeh, S., Solanki, S. K., Cameron, R. H., Barthol, P., Rodríguez, J. B., del Toro Iniesta, J. C., Gandorfer, A., Gizon, L., Hirzberger, J., Knölker, M., Pillet, V. M., Suárez, D. O., Riethmüller, T. L., Schmidt, W., and van Noort, M.: 2017, ApJS 229(1), 8

Jafarzadeh, S., Solanki, S. K., Feller, A., Lagg, A., Pietarila, A., Danilovic, S., Riethmüller, T. L., and Martínez Pillet, V.: 2013, A\&A 549, A116

Jafarzadeh, S., Solanki, S. K., Gafeira, R., Barthol, P., Berkefeld, T., Blanco Rodríguez, J., del Toro Iniesta, J. C., Gandorfer, A., Gizon, L., Hirzberger, J., Knölker, M., Martínez Pillet, V., Orozco Suárez, D., Riethmüller, T. L., Schmidt, W., and van Noort, M.: 2017, ApJS 229, 8

Jafarzadeh, S., Solanki, S. K., Lagg, A., Bellot Rubio, L. R., van Noort, M., Feller, A., and Danilovic, S.: 2014, A\&A 569, A105

Jafarzadeh, S., Solanki, S. K., Stangalini, M., Steiner, O., Cameron, R. H., and Danilovic, S.: 2017, ApJS 229, 9

Jess, D. B., Mathioudakis, M., Christian, D. J., Keenan, F. P., Ryans, R. S. I., and Crockett, P. J.: 2010, Sol. Phys. 261, 363

Jess, D. B., Mathioudakis, M., Erdélyi, R., Crockett, P. J., Keenan, F. P., and Christian, D. J.: 2009, Science 323, 1582

Jess, D. B., Morton, R. J., Verth, G., Fedun, V., Grant, S. D. T., and Giagkiozis, I.: 2015, Space Sci. Rev. 190, 103

Jess, D. B., Pascoe, D. J., Christian, D. J., Mathioudakis, M., Keys, P. H., and Keenan, F. P.: 2012, ApJ 744, L5

Jess, D. B. and Verth, G.: 2015, ArXiv e-prints 
Jones, H. P. and Giovanelli, R. G.: 1982, Sol. Phys. 79, 247

Judge, P.: 2006, in J. Leibacher, R. F. Stein, and H. Uitenbroek (eds.), Solar MHD Theory and Observations: A High Spatial Resolution Perspective, Vol. 354 of Astronomical Society of the Pacific Conference Series, p. 259

Judge, P. G. and Carlsson, M.: 2010, ApJ 719(1), 469

Judge, P. G., Tritschler, A., and Low, B. C.: 2011, ApJLetters 730(1), L4

Kahil, F., Riethmüller, T. L., and Solanki, S. K.: 2017, ApJS 229(1), 12

Kaithakkal, A. J., Riethmüller, T. L., Solanki, S. K., Lagg, A., Barthol, P., Gandorfer, A., Gizon, L., Hirzberger, J., vanNoort, M., Rodríguez, J. B., Iniesta, J. C. D. T., Suárez, D. O., Schmidt, W., Pillet, V. M., and Knölker, M.: 2017, ApJS 229(1), 13

Keller, C. U. and von der Lühe, O.: 1992, A\&A 261, 321

Khomenko, E. and Collados, M.: 2015, Living Rev. Solar Phys 12

Kitiashvili, I. N., Kosovichev, A. G., Mansour, N. N., and Wray, A. A.: 2011, ApJ 727, L50

Koza, J., Rutten, R. J., and Vourlidas, A.: 2009, A\&A 499, 917

Kukhianidze, V., Zaqarashvili, T. V., and Khutsishvili, E.: 2006, A\&A 449, L35

Kuridze, D., Morton, R. J., Erdélyi, R., Dorrian, G. D., Mathioudakis, M., Jess, D. B., and Keenan, F. P.: 2012, ApJ 750, 51

Lagg, A., Ishikawa, R., Merenda, L., Wiegelmann, T., Tsuneta, S., and Solanki, S. K.: 2009, in B. Lites, M. Cheung, T. Magara, J. Mariska, and K. Reeves (eds.), The Second Hinode Science Meeting: Beyond Discovery-Toward Understanding, Vol. $\overline{415}$ of Astronomical Society of the Pacific Conference Series, p. 327

Langangen, Ã., Pontieu, B. D., Carlsson, M., Hansteen, V. H., Cauzzi, G., and Reardon, K.: 2008, ApJLetters 679(2), L167

Leenaarts, J., Carlsson, M., Hansteen, V., and Rouppe van der Voort, L.: 2009, ApJ 694, L128

Leenaarts, J., Carlsson, M., and Rouppe van der Voort, L.: 2012, ApJ 749, 136

Leenaarts, J., Carlsson, M., and Rouppe van der Voort, L.: 2015, ApJ 802, 136

Leighton, R. B., Noyes, R. W., and Simon, G. W.: 1962, ApJ 135, 474

Lin, Y., Engvold, O., Rouppe van der Voort, L. H. M., and van Noort, M.: 2007, Sol. Phys. 246, 65

Lippincott, S. L.: 1957, Smithsonian Contributions to Astrophysics 2, 15 
Lites, B. W., Kubo, M., Socas-Navarro, H., Berger, T., Frank, Z., Shine, R., Tarbell, T., Title, A., Ichimoto, K., Katsukawa, Y., Tsuneta, S., Suematsu, Y., Shimizu, T., and Nagata, S.: 2008, ApJ 672, 1237

Liu, Y., Zhang, H., Ai, G., Wang, H., and Zirin, H.: 1994, A\&A 283, 215

Löfdahl, M. G.: 2002, in P. J. Bones, M. A. Fiddy, and R. P. Millane (eds.), Image

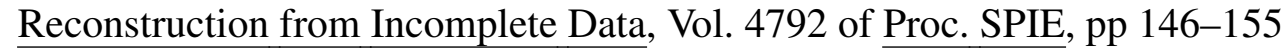

Löfdahl, M. G. and Scharmer, G. B.: 1994, ApJS. 107, 243-264 (1994) 107

Marsh, K. A.: 1976, Sol. Phys. 50, 37

Martínez Pillet, V., Del Toro Iniesta, J. C., Álvarez-Herrero, A., Domingo, V., Bonet, J. A., González Fernández, L., López Jiménez, A., Pastor, C., Gasent Blesa, J. L., Mellado, P., Piqueras, J., Aparicio, B., Balaguer, M., Ballesteros, E., Belenguer, T., Bellot Rubio, L. R., Berkefeld, T., Collados, M., Deutsch, W., Feller, A., Girela, F., Grauf, B., Heredero, R. L., Herranz, M., Jerónimo, J. M., Laguna, H., Meller, R., Menéndez, M., Morales, R., Orozco Suárez, D., Ramos, G., Reina, M., Ramos, J. L., Rodríguez, P., Sánchez, A., Uribe-Patarroyo, N., Barthol, P., Gandorfer, A., Knoelker, M., Schmidt, W., Solanki, S. K., and Vargas Domínguez, S.: 2011, Sol. Phys. 268, 57

Mathioudakis, M., Jess, D. B., and Erdélyi, R.: 2013, Space Sci. Rev. 175, 1

McIntosh, S. W., De Pontieu, B., Carlsson, M., Hansteen, V., Boerner, P., and Goossens, M.: 2011, Nature 475, 477

Michalitsanos, A. G.: 1973, Sol. Phys. 30, 47

Milić, I. and van Noort, M.: 2017, A\&A 601, A100

Moore, R. L., Sterling, A. C., Cirtain, J. W., and Falconer, D. A.: 2011, ApJ 731, L18

Mooroogen, K., Morton, R. J., and Henriques, V.: 2017, A\&A 607, A46

Morton, R. J., Erdélyi, R., Jess, D. B., and Mathioudakis, M.: 2011, ApJ 729, L18

Morton, R. J., Verth, G., Fedun, V., Shelyag, S., and Erdélyi, R.: 2013, ApJ 768, 17

Morton, R. J., Verth, G., Hillier, A., and Erdélyi, R.: 2014, ApJ 784, 29

Morton, R. J., Verth, G., Jess, D. B., Kuridze, D., Ruderman, M. S., Mathioudakis, M., and Erdélyi, R.: 2012, Nature Communications 3, 1315

Nakariakov, V. M., Hornsey, C., and Melnikov, V. F.: 2012, ApJ 761, 134

Nakariakov, V. M. and Verwichte, E.: 2005, Living Rev. Solar Phys. 2, 3. URL: http://www.livingreviews.org/lrsp

Nakariakov, V. M. and Verwichte, E.: 2005, Living Rev. Solar Phys 2(3)

Narayanan, A. S.: 2013, An introduction to waves and oscillations in the Sun, Springer 
Okamoto, T. J. and De Pontieu, B.: 2011, ApJ 736, L24

Okamoto, T. J., Tsuneta, S., Berger, T. E., Ichimoto, K., Katsukawa, Y., Lites, B. W., Nagata, S., Shibata, K., Shimizu, T., Shine, R. A., Suematsu, Y., Tarbell, T. D., and Title, A. M.: 2007, Science 318, 1577

Parker, E. N.: 1972, ApJ 174, 499

Pasachoff, J. M., Jacobson, W. A., and Sterling, A. C.: 2009, Sol. Phys. 260, 59

Pasachoff, J. M., Noyes, R. W., and Beckers, J. M.: 1968, Sol. Phys. 5(2), 131

Patsourakos, S., Gouttebroze, P., and Vourlidas, A.: 2007, ApJ 664, 1214

Paxman, R. G., Seldin, J. H., Loefdahl, M. G., Scharmer, G. B., and Keller, C. U.: 1996, ApJ 466, 1087

Pereira, T. M. D., De Pontieu, B., and Carlsson, M.: 2012, ApJ p. 17

Pereira, T. M. D., De Pontieu, B., Carlsson, M., Hansteen, V., Tarbell, T. D., Lemen, J., Title, A., Boerner, P., Hurlburt, N., Wülser, J. P., Martínez-Sykora, J., Kleint, L., Golub, L., McKillop, S., Reeves, K. K., Saar, S., Testa, P., Tian, H., Jaeggli, S., and Kankelborg, C.: 2014, ApJ 792, L15

Pereira, T. M. D., Rouppe van der Voort, L., and Carlsson, M.: 2016, ApJ 824, 65

Pereira, T. M. D., van der Voort, L. R., and Carlsson, M.: 2016, ApJ 824(2), 65

Pietarila, A., Cuadrado, R. A., Hirzberger, J., and Solanki, S. K.: 2011, Astrophys. J. 739(2), 92

Pietarila, A., Hirzberger, J., Zakharov, V., and Solanki, S. K.: 2009, A\&A 502(2), 647

Pietarila, A., Cameron, R., and Solanki, S. K.: 2010, A\&A 518, A50

Pizer, S. M., Amburn, E. P., Austin, J. D., Cromartie, R., Geselowitz, A., Greer, T., ter Haar Romeny, B., Zimmerman, J. B., and Zuiderveld, K.: 1987, Adaptive histogram equalization and its variations

Ploner, S. R. O. and Solanki, S. K.: 1999, A\&A 345, 986

Priest, E.: 2014, Magnetohydrodynamics of the Sun

Reardon, K. P., Cauzzi, G., and Rimmele, T.: 2007, in P. Heinzel, I. Dorotovič, and R. J. Rutten (eds.), The Physics of Chromospheric Plasmas, Vol. 368 of Astronomical Society of the Pacific Conference Series, p. 151

Requerey, I. S., Cobo, B. R., Iniesta, J. C. D. T., Suárez, D. O., Rodríguez, J. B., Solanki, S. K., Barthol, P., Gandorfer, A., Gizon, L., Hirzberger, J., Riethmüller, T. L., van Noort, M., Schmidt, W., Pillet, V. M., and Knölker, M.: 2017a, ApJS 229(1), 15

Requerey, I. S., Iniesta, J. C. D. T., Rubio, L. R. B., Pillet, V. M., Solanki, S. K., and Schmidt, W.: 2017b, ApJS 229(1), 14 
Riethmüller, T. L., Solanki, S. K., Barthol, P., Berkefeld, T., Blanco Rodríguez, J., del Toro Iniesta, J. C., Gandorfer, A., Gizon, L., Hirzberger, J., Knölker, M., Martínez Pillet, V., Orozco Suárez, D., Riethmüller, T. L., Schmidt, W., and van Noort, M.: 2017, ApJS 229, 15

Riethmüller, T. L., Solanki, S. K., Berdyugina, S. V., Schüssler, M., Martínez Pillet, V., Feller, A., Gandorfer, A., and Hirzberger, J.: 2014, A\&A 568, A13

Rimmele, T. R. and Marino, J.: 2011, Living Rev. Solar Phys 8(1), 2

Roberts, B.: 2004, in Lacoste, H. (ed.), SOHO 13 Waves, Oscillations and Small-Scale Transients Events in the Solar Atmosphere: Joint View from SOHO and TRACE, Vol. 547 of ESA Special Pub. (Paris: ESA), p. 1

Roberts, W. O.: 1945, ApJ 101, 136

Rouppe van der Voort, L., Leenaarts, J., de Pontieu, B., Carlsson, M., and Vissers, G.: 2009, ApJ 705, 272

Rouppe van der Voort, L. H. M., De Pontieu, B., Hansteen, V. H., Carlsson, M., and van Noort, M.: 2007, ApJ 660, L169

Rutten, R. J.: 2007, in Heinzel, P. and Dorotovič, I. and Rutten, R. J. (ed.), The Physics of Chromospheric Plasmas, Vol. 368 of ASP Conf. Ser. (San Francisco: ASP), p. 27

Rutten, R. J.: 2012, Royal Society of London Philosophical Transactions Series A 370, 3129

Rutten, R. J., de Wijn, A. G., and Sütterlin, P.: 2004, A\&A 416, 333

Saha, S. K.: 2007, Diffraction-Limited Imaging With Large And Moderate Telescopes

Sawyer, C.: 1972, Sol. Phys. 24, 79

Scharmer, G. B., Bjelksjo, K., Korhonen, T. K., Lindberg, B., and Petterson, B.: 2003a, in S. L. Keil and S. V. Avakyan (eds.), Innovative Telescopes and Instrumentation for Solar Astrophysics, Vol. 4853 of Proc. SPIE, pp 341-350

Scharmer, G. B., Bjelksjo, K., Korhonen, T. K., Lindberg, B., and Petterson, B.: 2003b, in S. L. Keil and S. V. Avakyan (eds.), Society of Photo-Optical Instrumentation Engineers (SPIE) Conference Series, Vol. 4853 of SPIE Conf. Ser., p. 341

Scharmer, G. B., Narayan, G., Hillberg, T., de la Cruz Rodriguez, J., Löfdahl, M. G., Kiselman, D., Sütterlin, P., van Noort, M., and Lagg, A.: 2008, ApJ 689, L69

Schmidt, W., von der Lühe, O., Volkmer, R., Denker, C., Solanki, S. K., Balthasar, H., Bello Gonzalez, N., Berkefeld, T., Collados, M., Fischer, A., Halbgewachs, C., Heidecke, F., Hofmann, A., Kneer, F., Lagg, A., Nicklas, H., Popow, E., Puschmann, K. G., Schmidt, D., Sigwarth, M., Sobotka, M., Soltau, D., Staude, J., Strassmeier, K. G., and Waldmann , T. A.: 2012, Astronomische Nachrichten 333, 796 
Schrijver, C. J., Hagenaar, H. J., and Title, A. M.: 1997, ApJ 475, 328

Schüssler, M.: 1986, in Deinzer, W. and Knölker, M. and Voigt, H. H. (Göttingen: Vandenhoeck and Ruprecht) (ed.), Small Scale Magnetic Flux Concentrations in the Solar Photosphere, p. 103

Schüssler, M., Caligari, P., Ferriz-Mas, A., and Moreno-Insertis, F.: 1994, A\&A 281, L69

Sekse, D. H., Rouppe van der Voort, L., and De Pontieu, B.: 2013, ApJ 764, 164

Shine, R. A., Title, A. M., Tarbell, T. D., Smith, K., Frank, Z. A., and Scharmer, G.: 1994, ApJ 430, 413

Smitha, H. N., Anusha, L. S., Solanki, S. K., and Riethmüller, T. L.: 2017, ApJS 229, 17

Socas-Navarro, H., Ruiz Cobo, B., and Trujillo Bueno, J.: 1998, ApJ 507, 470

Solanki, S. K.: 1993a, Space Sci. Rev. 63, 1

Solanki, S. K.: 1993b, Space Sci. Rev. 63, 1

Solanki, S. K., Barthol, P., Danilovic, S., Feller, A., Gandorfer, A., Hirzberger, J., Riethmüller, T. L., Schüssler, M., Bonet, J. A., Pillet, V. M., del Toro Iniesta, J. C., Domingo, V., Palacios, J., Knölker, M., González, N. B., Berkefeld, T., Franz, M., Schmidt, W., and Title, A. M.: 2010, ApJ 723(2), L127

Solanki, S. K., Inhester, B., and Schüssler, M.: 2006, Rep. Prog. in Phys. 69, 563

Solanki, S. K., Riethmüller, T. L., Barthol, P., Danilovic, S., Deutsch, W., Doerr, H. P., Feller, A., Gandorfer, A., and Sunrise team: 2017, ApJS 229, 1

Solanki, S. K. and Steiner, O.: 1990, A\&A 234, 519

Solanki, S. K., Steiner, O., and Uitenbroeck, H.: 1991, A\&A 250, 220

Spruit, H. C.: 1976, Sol. Phys. 50, 269

Spruit, H. C.: 1981, A\&A 98, 155

Spruit, H. C.: 1982, Sol. Phys. 75, 3

Srivastava, A. K., Zaqarashvili, T. V., Uddin, W., Dwivedi, B. N., and Kumar, P.: 2008, MNRAS 388, 1899

Stangalini, M., Giannattasio, F., and Jafarzadeh, S.: 2015, A\&A 577, A17

Stenflo, J. O.: 1973, Sol. Phys. 32, 41

Sterling, A. C.: 2000, Sol. Phys. 196(1), 79

Su, J. T., Shen, Y. D., Liu, Y., Liu, Y., and Mao, X. J.: 2012, ApJ 755, 113

Suematsu, Y., Wang, H., and Zirin, H.: 1995, ApJ 450, 411 
Tanaka, K.: 1974, in R. G. Athay (ed.), Chromospheric Fine Structure, Vol. 56 of IAU Symposium, p. 239

Tomczyk, S., McIntosh, S. W., Keil, S. L., Judge, P. G., Schad, T., Seeley, D. H., and Edmondson, J.: 2007, Science 317, 1192

Torrence, C. and Compo, G. P.: 1998, BAMS 79, 61

Tritschler, A., Rimmele, T. R., Berukoff, S., Casini, R., Kuhn, J. R., Lin, H., Rast, M. P., McMullin, J. P., Schmidt, W., Wöger, F., and DKIST Team: 2016, Astronomische Nachrichten 337, 1064

Tsiropoula, G., Tziotziou, K., Kontogiannis, I., Madjarska, M. S., Doyle, J. G., and Suematsu, Y.: 2012, Space Sci. Rev. 169, 181

Tsuneta, S., Ichimoto, K., Katsukawa, Y., Nagata, S., Otsubo, M., Shimizu, T., Suematsu, Y., Nakagiri, M., Noguchi, M., Tarbell, T., Title, A., Shine, R., Rosenberg, W., Hoffmann, C., Jurcevich, B., Kushner, G., Levay, M., Lites, B., Elmore, D., Matsushita, T., Kawaguchi, N., Saito, H., Mikami, I., Hill, L. D., and Owens, J. K.: 2008, Sol. Phys. 249, 167

van Ballegooijen, A. A., Asgari-Targhi, M., Cranmer, S. R., and DeLuca, E. E.: 2011, ApJ 736, 3

Van Doorsselaere, T., De Groof, A., Zender, J., Berghmans, D., and Goossens, M.: 2011, ApJ 740, 90

Van Doorsselaere, T., Gijsen, S. E., Andries, J., and Verth, G.: 2014, ApJ 795, 18

Van Doorsselaere, T., Nakariakov, V. M., and Verwichte, E.: 2008, ApJ 676, L73

van Noort, M., van Der Voort, L. R., and Löfdahl, M. G.: 2005, Sol. Phys. 228(1-2), 191

Vecchio, A., Cauzzi, G., Reardon, K. P., Janssen, K., and Rimmele, T.: 2007, A\&A 461, L1

Vernazza, J. E., Avrett, E. H., and Loeser, R.: 1981, ApJS 45, 635

Verwichte, E., Nakariakov, V. M., Ofman, L., and Deluca, E. E.: 2004, Sol. Phys. 223, 77

Vissers, G. and Rouppe van der Voort, L.: 2012, ApJ 750, 22

Vögler, A. and Schüssler, M.: 2007, A\&A 465, L43

Wang, H., Johannesson, A., Stage, M., Lee, C., and Zirin, H.: 1998, Sol. Phys. 178(1), 55

Wang, J., Wang, H., Tang, F., Lee, J. W., and Zirin, H.: 1995, Sol. Phys. 160, 277

Wang, T., Solanki, S. K., Curdt, W., Innes, D. E., and Dammasch, I. E.: 2002, ApJ 574, L101

Wang, T. J. and Solanki, S. K.: 2004, A\&A 421, L33 
Wedemeyer, S., Freytag, B., Steffen, M., Ludwig, H.-G., and Holweger, H.: 2004, A\&A 414, 1121

Wedemeyer-Böhm, S., Lagg, A., and Nordlund, A.: 2008, Space Sci. Rev. 144(1-4), 317

Wiegelmann, T., Neukirch, T., Nickeler, D. H., Solanki, S. K., Barthol, P., Gandorfer, A., Gizon, L., Hirzberger, J., Riethmüller, T. L., van Noort, M., Blanco Rodríguez, J., del Toro Iniesta, J. C., Orozco Suárez, D., Schmidt, W., Martínez Pillet, V., and Knölker, M.: 2017, ApJS 229, 17

Wiegelmann, T., Solanki, S. K., Borrero, J. M., Martínez Pillet, V., del Toro Iniesta, J. C., Domingo, V., Bonet, J. A., Barthol, P., Gandorfer, A., Knölker, M., Schmidt, W., and Title, A. M.: 2010, ApJ 723, L185

Withbroe, G. L. and Noyes, R. W.: 1977, ARA\&A 15, 363

Wöger, F., Wedemeyer-Böhm, S., Uitenbroek, H., and Rimmele, T. R.: 2009, ApJ 706(1), 148

Zaqarashvili, T. V. and Erdélyi, R.: 2009, Space Sci. Rev. 149, 355

Zaqarashvili, T. V., Khutsishvili, E., Kukhianidze, V., and Ramishvili, G.: 2007, A\&A 474, 627

Zirin, H.: 1974, Sol. Phys. 38, 91 
Appendix 



\section{A Transverse Oscillations in Slender Ca II H Fibrils Observed with SUNRISE/SUFI}

S. Jafarzadeh, S. K. Solanki, R. Gafeira, M. van Noort, P. Barthol, J. Blanco Rodríguez, J. C. del Toro Iniesta, A. Gandorfer, L. Gizon, J. Hirzberger, M. Knölker, D. Orozco Suárez, T. L. Riethmüller, and W. Schmidt published in ApJS, 229, 2017, DOI 10.3847/1538-4365/229/1/9. Reproduced with permission of ApJS

\section{Abstract}

We present observations of transverse oscillations in slender Ca II H fibrils (SCFs) in the lower solar chromosphere. We use a $1 \mathrm{hr}$ long time series of high- (spatial and temporal-) resolution seeing-free observations in a $1.1 \AA$ wide passband covering the line core of Ca II H $3969 \AA$ from the second flight of the SUNRISE balloon-borne solar observatory. The entire field of view, spanning the polarity inversion line of an active region close to the solar disk center, is covered with bright, thin, and very dynamic fine structures. Our analysis reveals the prevalence of transverse waves in SCFs with median amplitudes and periods on the order of $2.4 \pm 0.8 \mathrm{~km} \mathrm{~s}^{-1}$ and $83 \pm 29 \mathrm{~s}$, respectively (with standard deviations given as uncertainties). We find that the transverse waves often propagate along (parts of) the SCFs with median phase speeds of $9 \pm 14 \mathrm{~km} \mathrm{~s}^{-1}$. While the propagation is only in one direction along the axis in some of the SCFs, propagating waves in both directions, as well as standing waves are also observed. The transverse oscillations are likely Alfvénic and are thought to be representative of magnetohydrodynamic kink waves. The wave propagation suggests that the rapid high-frequency transverse waves, often produced in the lower photosphere, can penetrate into the chromosphere with an estimated energy flux of $\approx 15 \mathrm{~kW} \mathrm{~m}^{-2}$. Characteristics of these waves differ from those reported for other fibrillar structures, which, however, were observed mainly in the upper solar chromosphere.

\section{A.1 Introduction}

The solar chromosphere is a highly structured environment exhibiting elongated features at different spatial scales (Judge 2006; Rutten 2007; Wöger et al. 2009). Depend- 
ing on their physical and dynamical properties, these thread-like structures have been given names such as fibrils, dynamic fibrils, mottles, straws, rosettes, spicules, or rapid blue/redshifted events (RBEs/RREs) in the literature (De Pontieu et al. 2007; Rutten 2007; Rouppe van der Voort et al. 2009); see Rutten (2012); see also Tsiropoula et al. (2012) for a recent review. Most of these structures often share some properties and appear to be associated with photospheric concentrations of the magnetic field such as magnetic bright points and plages. These features have been mostly observed in $\mathrm{H} \alpha 6563 \AA$ and Ca II $8542 \AA$ images (representing the mid-to-upper chromosphere) on the solar disk (Vecchio et al. 2007; Cauzzi et al. 2008), and in Ca II $8542 \AA$, Ca II H/K, and $\mathrm{H} \alpha$ images off the limb (e.g., Pasachoff et al. (2009); Pereira et al. (2016); see Pereira et al. (2014)).

In particular, internetwork fibrils (usually seen in $\mathrm{H} \alpha$ images) are relatively long and are often considered to outline the magnetic field lines in the chromosphere. They appear to constitute magnetic canopies in the mid-to-upper chromosphere, along which flows may reach to the transition region (with some signatures observed in, e.g., He II $304 \AA$; Rutten (2012)). Their correspondence to the chromospheric magnetic field topology has been partly confirmed by comparisons with full Stokes observations in Ca II $8542 \AA$ (de la Cruz Rodríguez and Socas-Navarro 2011). These elongated features are, however, shorter and more dynamic in and close to network and active regions and are sometimes seen extending into the upper atmosphere (Suematsu et al. 1995). The most dynamic of the active region elongated features are jet-like features seen in, e.g., Ly $\alpha$ observations (Patsourakos et al. 2007; Koza et al. 2009).

From observations in a $0.3 \AA$ wide Ca II K passband, Zirin (1974) claimed the existence of $\mathrm{Ca}$ II $\mathrm{K}$ bright fibrils matching dark fibrils in $\mathrm{H} \alpha$ filtergrams. Lifetime and length of those fibrils was thought to depend on the inclination of the field lines (Marsh 1976).

Investigating the on-disk $\mathrm{Ca}$ II $\mathrm{H} \& \mathrm{~K}$ bright fibrils (in the lower chromospheric layers) has remained a challenging task. First, these very thin and bright features can only be identified in relatively narrowband Ca II H\&K filters (Reardon et al. 2007; Beck et al. 2013). Thus, broader filters, such as that of Hinode/SOT Tsuneta et al. (2008); FWHM of $3.0 \AA$ ), sample a wide range in formation height, from the mid photosphere (in their wings), at around the temperature minimum at the $\mathrm{H}_{1} / \mathrm{K}_{1}$ dip, reaching up to the mid chromosphere at the $\mathrm{H}_{2} / \mathrm{K}_{2}$ emission peaks around their line-cores (Vernazza et al. 1981). Hence, the Hinode/SOT Ca II H filter is too broad to see the slender bright fibrils in the lower chromosphere. In addition, precise detection of the very thin and dynamic $\mathrm{Ca}$ II $\mathrm{H} / \mathrm{K}$ fibrils needs data with high spatial and temporal resolution (Pietarila et al. 2009).

Second, ground-based observations of temporal evolution of these fine structures with, e.g., the narrowband Ca II H filter (FWHM $\approx 1.0 \AA$ ) of the Swedish Solar Telescope (SST; Scharmer et al. 2003a), need exceptional seeing conditions for a relatively long duration.

The only thorough study of such slender bright fibrils, to our knowledge, was provided by Pietarila et al. (2009) who demonstrated that these thin structures are ubiquitous in high spatial resolution and high-quality time series of images of a small active region recorded in a $1.5 \AA \mathrm{Ca}$ II H\&K filter with the SST. We note that fibril-like structures (often dark) above sunspots have been also reported in observations of the SST narrowband Ca II $\mathrm{H}$ filter (Henriques and Kiselman 2013; Henriques et al. 2015).

A range of waves and oscillations that are thought to be important for heating the upper solar atmosphere have been observed in these fibrillar structures (e.g., Lin et al. 2007; Pietarila et al. 2011; Tsiropoula et al. 2012, for a review). These waves are often produced 
in the photosphere by, e.g., the buffeting of footpoints of their underlying magnetic elements, and propagate (longitudinally or transversely) along the magnetic field lines on different scales. The fibrils appear to act as a guide for the propagation of the MHD waves (Hansteen et al. 2006; De Pontieu et al. 2007) whose leakage to the upper solar atmosphere depends on, e.g., the inclination angle of the fibrils (Michalitsanos 1973; Bel and Leroy 1977). Thus, dissipation of energy carried by these MHD waves (of different types) is of interest for the energy budget at different heights in the chromosphere and in the corona ( De Pontieu et al. (2011), also see Jess et al. (2015) for a recent review).

In particular, transverse waves (in both standing and propagating states) have been observed in a number of features throughout the solar atmosphere, such as in magnetic elements in the lower solar atmosphere (e.g., Stangalini et al. 2015; Jafarzadeh et al. 2017), in spicules and filaments in the upper chromosphere (e.g., De Pontieu et al. 2007; Lin et al. 2007), and in prominences and loops in the corona (e.g., Aschwanden et al. 1999; Okamoto et al. 2007). These transverse waves have often been interpreted as MHD kink and/or Alfvén waves (Spruit 1982; Nakariakov and Verwichte 2005; Erdélyi et al. 2007). To date, however, these transverse waves have not been fully quantified in the lower solar chromosphere (e.g., in Ca II H/K fibrils).

In this paper, we present ubiquitous transverse oscillations in slender $\mathrm{Ca}$ II $\mathrm{H}$ fibrils (SCFs) from a high-quality and relatively long time series of images recorded at high spatial and temporal resolution (in a $1.1 \AA$ narrowband filter) with the SUNRISE balloonborne solar observatory. We quantify the oscillations by analyzing the swaying motions in several spatial locations along their axes in the course of their lifetimes. We provide parameters of the oscillations/waves and suggest the role they may play in the localized heating of the lower solar chromosphere.

\section{A.2 Observations}

Our analysis is based on high-resolution images in the Ca II $\mathrm{H}$ narrowband filter (FWHM $\approx 1.1 \AA$ ) acquired with the SUNRISE Filter Imager (SUFI; Gandorfer et al. 2011) aboard the 1-meter SUNRISE balloon-borne solar observatory (Solanki et al. 2010; Barthol et al. 2011; Berkefeld et al. 2011) in June 2013, i.e., its second flight (see Solanki et al. 2017, for more details on the second flight of SUNRISE). The seeing-free, uninterrupted time series of images were recorded for $\approx 60$ minutes (between 23:39 UT on 2013 June 12 and 00:38 UT on 2013 June 13) with a cadence of $7 \mathrm{~s}$. The Ca II H filtergrams cover a field of view $(F O V)$ of $(15 \times 38) \operatorname{arcsec}^{2}$, after image restoration (Hirzberger et al. $2010,2011)$, in the immediate vicinity of an active region close to the solar disk center (with the cosine of heliocentric angle $\mu \approx 0.93$ ). The images include a few small pores and plages in their FOVs. These high-quality data were further corrected for wavefront aberrations by means of multi-frame blind deconvolution (van Noort et al. 2005). Finally, the time series of images were corrected for field rotation by determining the rotation angle from the telescope pointing model (which depends on the position of the Sun on the sky). Temporal misalignments were also corrected for, which included determining the geometrical shift and scale of each frame with respect to its preceding image, using a cross-correlation technique. There could still, however, remain residual alignment errors between consecutive frames due to, e.g., some jitter from the telescope. Following Shine 
et al. (1994), we employed a de-stretching approach to remove the variable distortion of the images. In short, each frame was divided into a grid of overlapping sub-images whose spatial misalignments with their corresponding ones in the following image were determined and corrected.

We note that in addition to the line core (sampling the low-to-mid chromospheric heights), the 1.1 $\AA \mathrm{Ca}$ II $\mathrm{H}$ passband also includes a small portion of the inner line wings contaminated by photospheric radiation. However, in the presence of strong magnetic fields, the contribution of the line core (through further enhancement of the emission peaks) is much larger than that of the wings (Beck et al. 2013). Hence, our images mainly represent chromospheric heights.

Additionally, the SUNRISE Imaging Magnetograph eXperiment (IMaX; Martínez Pillet et al. 2011) undertook simultaneous full Stokes (I, Q, U, and V) observations of the magnetically sensitive Fe I 5250.2 A line, which overlap with the first $\approx 17$ minutes of our $\mathrm{Ca}$ II H observations. The IMaX images cover a larger FOV of $(51 \times 51) \operatorname{arcsec}^{2}$ and are used to identify the magnetic areas based on Stokes V observations.

Figure A.1 illustrates an example of the SUFI Ca II H image in a selected time stamp. Its co-spatial and co-temporal Stokes V image in the IMaX Fe i 5250.2 $\AA$ (an average over four wavelength positions at \pm 80 and $\pm 40 \mathrm{~m} \AA$ from the line center), as well as its co-temporal Stokes I continuum frame (but with the full FOV of IMaX), are also plotted in panels (b) and (c), respectively. The FOV of the images in panels (a) and (b) is marked with the red dashed box on the Stokes I continuum image. The yellow box in panel (a) includes the region of interest (ROI) whose analysis is discussed in Section 3. The ROI is chosen to include a region with a high density of fibrils, but excluding the pores. We note that the FOV of the images in Figure 1 is vertically flipped and slightly rotated with respect to the true orientation on the Sun. For the correct orientation, see Solanki et al. (2017).

\section{A.3 Analysis and Results}

The study of individual SCFs embedded in the spatially dense forest of bright, extremely thin, and highly dynamic fibrillar structures observed in the SUFI narrowband Ca II H image sequences needs several steps of image processing prior to the actual analysis. It is specially important since, in addition to these bright structures, $\mathrm{Ca}$ II $\mathrm{H}$ images also exhibit extended bright background and/or other brightenings due to, e.g., shock waves and/or reversed granulation. We note that the latter has, however, less impact on our images, whose observations in a relatively narrowband filter within an active region sample higher chromospheric layers (with less imprint of the reversed granulation) compared to those in the quiet Sun (see, e.g., Riethmüller et al. 2014).

After the image processing (described in the following subsection), the SCFs are then segmented using a semi-automated procedure. This is followed by tracing the detected SCFs in the times series of images and investigating possible transverse oscillations along their axes. These oscillations are further quantified by determining their amplitudes, periods, and transverse velocities. Phase speeds of propagating waves are also calculated, when possible. 

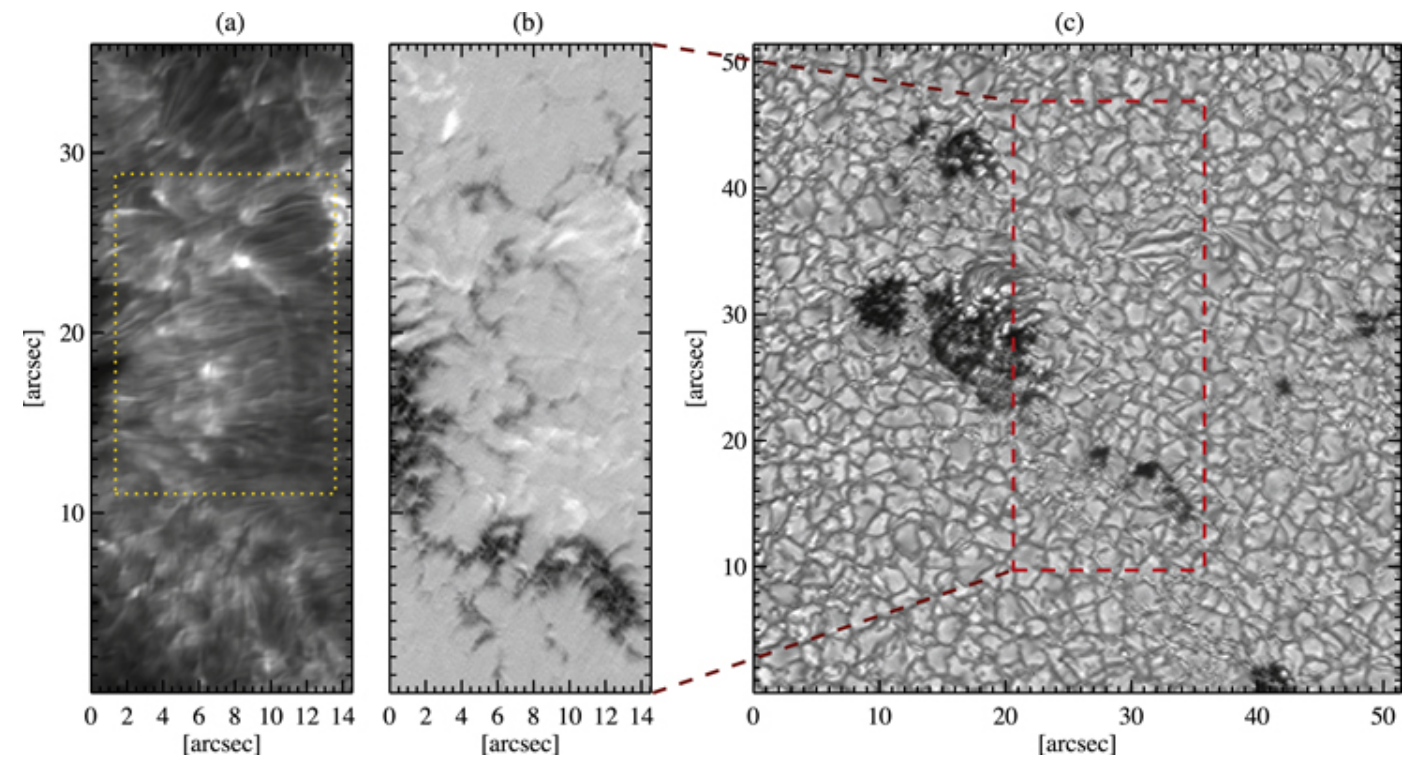

Figure A.1: Example of the SUNRISE/SUFI CaIIH narrowband image (a), the cotemporal, co-aligned Stokes V image from SUNRISE/IMaX cropped to the SUFI field of view (b), and the corresponding Stokes I continuum image at its original size and resolution (c). The red dashed-line box in panel (c) indicates the same field of view as those in panels (a) and (b). The yellow box in panel (a) marks a region of interest for which results of image restorations are illustrated in Figure A.2.

\section{A.3.1 Image Restoration and Fibril Identification}

In order to facilitate the identification of the bright SCFs in extended bright regions sampled with the $\mathrm{Ca}$ II $\mathrm{H}$ passband, we restored the images based on a process described by Jafarzadeh (2013). For discrete images, this approach minimizes inhomogeneities due to non-uniform background solar intensity, noise, and geometric distortion produced by slightly rectangular pixels. The image restoration employs a real-space spatial bandpass filter to eliminate localized extended brightenings (i.e., low spatial frequencies) caused by, e.g., shock waves, followed by corrections for high-frequency noise.

The localized and elongated features in the resulting images possess enhanced intensity profiles that are proportional to their original brightness. These include, however, small structures that are generally not SCFs (e.g., $\mathrm{H}_{2 V}$ grains or magnetic bright points; see Carlsson and Stein (1997) and Jafarzadeh et al. (2013)) and/or spurious artifacts introduced by the image restoration. Such features are discarded in the next steps.

An example of a Ca II H image for the ROI (marked in Figure A.1(a) with a yellow box) and its restored image are shown in Figure A.2(a) and (b), respectively.

The small, bright features that are not of interest for the present study are then detected and removed from each restored image. This is implemented using a modified "blob analyzer" algorithm, where all isolated elongated features in a bi-level image (i.e., a binary of the restored image) are automatically identified. All detected features are given a unique identity and their areas as well as their (major and minor) axes are determined by fitting ellipses to the features. Structures with major axes shorter than 2 arcsec are excluded from the images. Illustrated in Figure A.2(c) and (d) are the inverted binary version of 
(a)

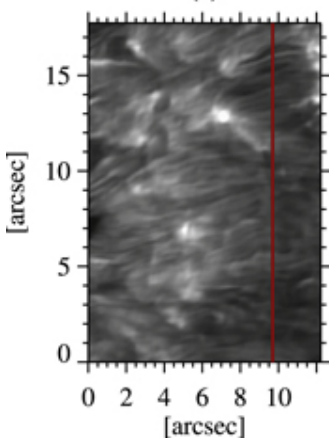

(b)

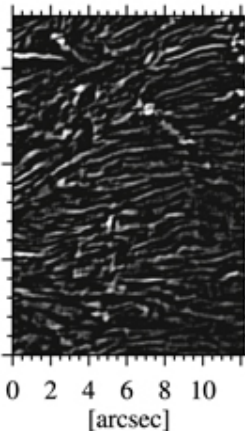

(c)

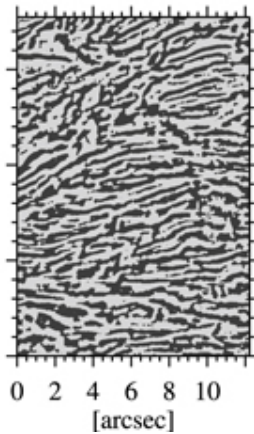

(d)

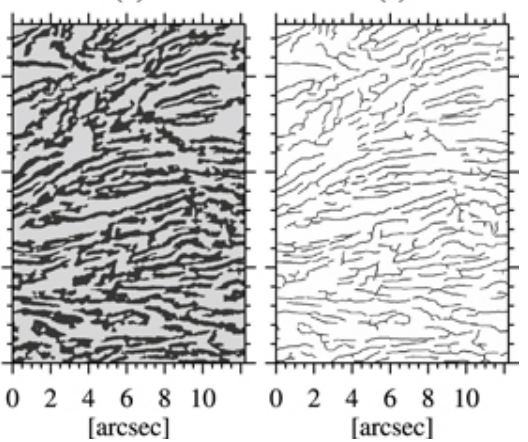

Figure A.2: Segmentation and modeling of slender Ca II H fibrils (SCFs) in the region of interest indicated by the yellow box in Figure 1(a). (a) Original intensity image. (b) Restored image, where the non-uniform, extended background intensity of the original image has been subtracted. The brightness is enhanced for better visibility. (c) Inverted binary image of panel (b). (d) Same as panel (c), except that particularly small and short features have been removed from the image. (e) Skeleton of the features shown in panel (d). The red line in panel (a) marks a cut through the image, along which displacement oscillations are studied (see Figure A.3).

the restored image shown in Figure A.2(b), before and after removal of small features, respectively. We refer to the latter as the "clean image" in the following. Furthermore, spines of the elongated features are depicted in Figure A.2(e). These skeletons are found as the median axes of the features in Figure A.2(d) and represent the overall shape of the SCFs regardless of their thickness. It turned out that the majority of the SCFs, as evidenced by the skeletons in Figure A.2(e), are oriented along the X-axis (sometimes with a small angle).

Identifying SCFs from the clean images still needs careful human intervention. This is particularly important since there are features composed of several bright events (including individual fibrils) superposed on a small area. Thus, the time series of clean images are visually inspected. Features meeting the following criteria are manually selected as our SCF candidates. (1) They can be visually observed in at least three consecutive frames (to avoid false detections as a result of, e.g., artifacts). (2) They are, to a large extent, isolated, i.e., their edges are clearly seen in the course of their lifetimes and no other significant brightenings occur at their spatial locations. In this manner interacting fibrils are excluded.

The SCFs appear not to move over a large distance during their relatively short apparent lifetimes, which are likely lower limits of their true lifetimes (see Gafeira et al. 2017b). Instead, they sway, more or less, around the same location in the time series of images. We use the CRISPEX tool (Vissers and Rouppe van der Voort 2012) for the visual inspections; since it provides not only the ease of browsing image sequences, but also spacetime plots for selected spatial locations, with different lengths and orientations. The latter is particularly convenient for identifying the isolated SCFs. 
(a)

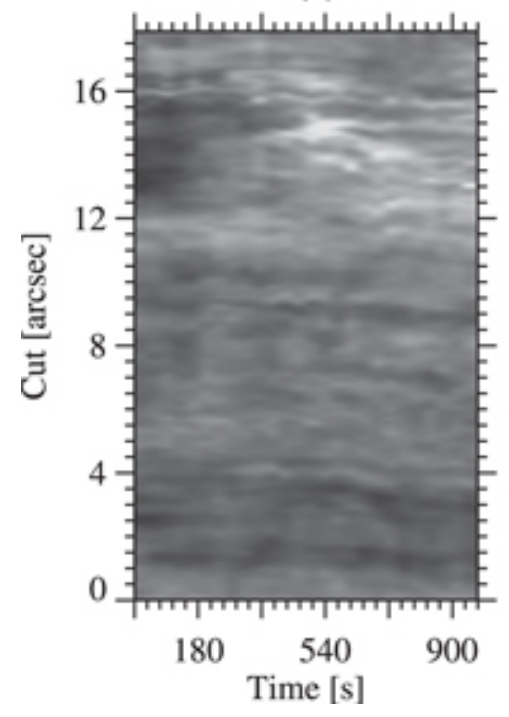

(b)

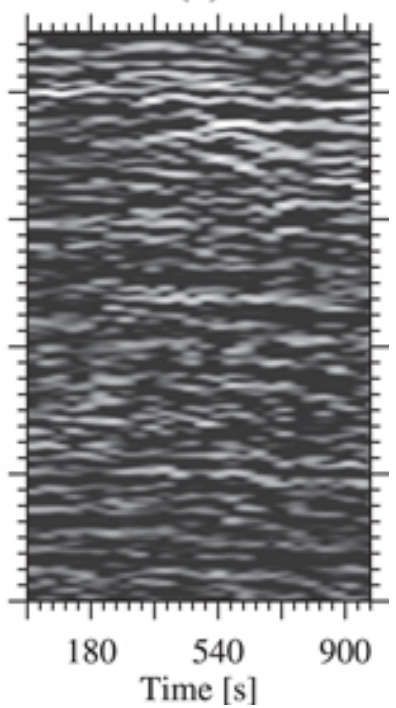

Figure A.3: Illustration of ubiquity of transverse oscillations in the slender Ca II $\mathrm{H}$ fibrils (SCFs). (a) A spacetime plot along a cut depicted with the red line in Figure A.2(a). (b) Same as (a), but obtained from the corresponding restored images, with the brightness enhanced for better visibility.

\section{A.3.2 Transverse Oscillations}

\section{A.3.2.1 Ubiquity}

To inspect transverse oscillations in the SCFs, we create artificial slits oriented more or less perpendicular to the SCFs axes at any given time. Spacetime plots are then extracted by stacking the slits at every time step (i.e., every $7 \mathrm{~s}$ ). These plots show the intensity variations with time along the slits. Figure A.3(a) illustrates a spacetime plot of the SCFs along a cut perpendicular to the axes of the majority of the linear features in our ROI, following the red line in Figure A.2(a). It clearly reveals transverse oscillations, i.e., displacements, normal to the spines of the SCFs. Because of the substantial superposition of various bright features in the $\mathrm{CaII} \mathrm{H}$ images, the ubiquitous transverse motions are best seen in a spacetime plot from the restored/clean images in which the background solar intensity and noise are diminished (Figure A.3(b)). As noted in Section 3.1, most of the SCFs in our ROI (Figure A.2 (a)) are along the x-axis, thus, the cut has been chosen to be in y direction for the example shown in Figure A.3. For simplicity, and better visibility of the wave patterns, the variations are plotted for the first quarter of the time series of images in our data set.

While the majority of the identified SCFs can be followed only for a relatively short time (i.e., shorter than $\approx 100 \mathrm{~s}$ ), events that are consistently visible longer undergo periodic oscillations normal to the SCFs' spines. These may represent transverse waves in the fibrils. In addition, we can also note the presence of some static bright features, which may represent non-oscillatory SCFs (or SCFs with small-amplitude oscillations that are not detected). We note that those brightenings may, however, also represent other non-SCF events. 


\section{A.3.2.2 Characteristics}

We determine period, displacement, and transverse velocity of identified oscillations from the spacetime diagrams. The oscillations are visually selected in a two-step procedure: (1) wave-like patterns are pre-identified from spacetime plots, similar to those shown in Figure 3, but along cuts perpendicular to individual detected fibrils. (2) Fibrils corresponding to the wave patterns are visually inspected in the time series of images (from both original and restored/clean images) in a small area around the SCFs. The latter confirms whether the wave-like patterns, identified in the first step, truly correspond to transverse oscillations in isolated SCFs, i.e., if they appear to sway in the image sequence played as a movie.

The selected wave patterns are then quantified using a semi-automated procedure where spatial locations of the oscillations are determined (see, e.g., Aschwanden et al. 2002; Verwichte et al. 2004; Morton et al. 2014, for similar techniques). Several points are visually selected on the approximate centroid of each oscillation at fixed cadence. This facilitates automatic measurements of the precise location of the spine by fitting a Gaussian at the selected points, perpendicular to the axis of the wave pattern in the spacetime diagram. The Gaussian fits provide us with the locations and widths of the SCF along the cut at different times. The locations were determined using the same algorithm as introduced by Jafarzadeh (2013), which technically has a precision of 0.05 pixel (better than $1 \mathrm{~km})$. However, in accordance with a discussion by Jafarzadeh et al. (2013) and Jafarzadeh et al. (2014), we consider a more conservative uncertainty of 0.5 pixel $(\approx 7$ $\mathrm{km})$.

Figures 4(a) and (b) represent a small area including a sample SCF (i.e., in the original and restored images, respectively). Transverse oscillation of the SCF along the cut marked in yellow in panels (a) and (b) is shown in panel (c). Width (the vertical blue lines) and the centroid of the oscillation (the red line; smoothed by a box average of 0.05 arcsec width in space and $5 \mathrm{~s}$ width in time), determined from a Gaussian fit at the equally spaced locations along the SCF, are also illustrated.

We note that the measurements are only implemented on oscillations lasting at least one full period. Prior to the analysis of each oscillation, we detrend the wave pattern to remove linear (systematic) drift. Then, following Leenaarts et al. (2015), periods $(P)$, transverse displacements $(D)$, and transverse velocities $(v)$ are calculated as

$$
\begin{gathered}
P_{i}=t_{i+1}-t_{i-1}, \\
D_{i}=\frac{\left|x_{i+1}-x_{i}\right|}{2}, \\
v_{i}=\frac{\pi\left(D_{i-1}+D_{i}\right.}{p_{i}},
\end{gathered}
$$

where $x$ and $t$, respectively, represent the space and time coordinates of the ith extremum of the oscillation, whereby $x$ is chosen to lie perpendicular to the time-averaged spine of the SCF. 
(a)

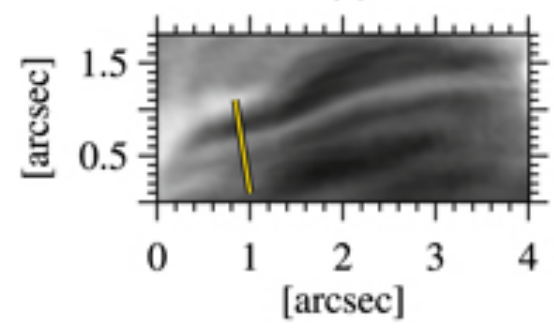

(b)

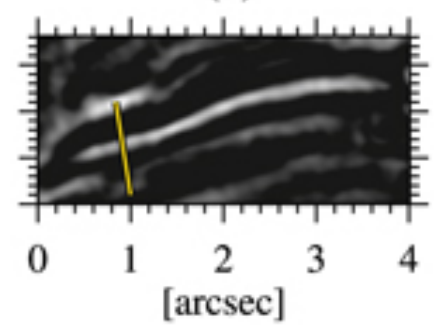

(c)

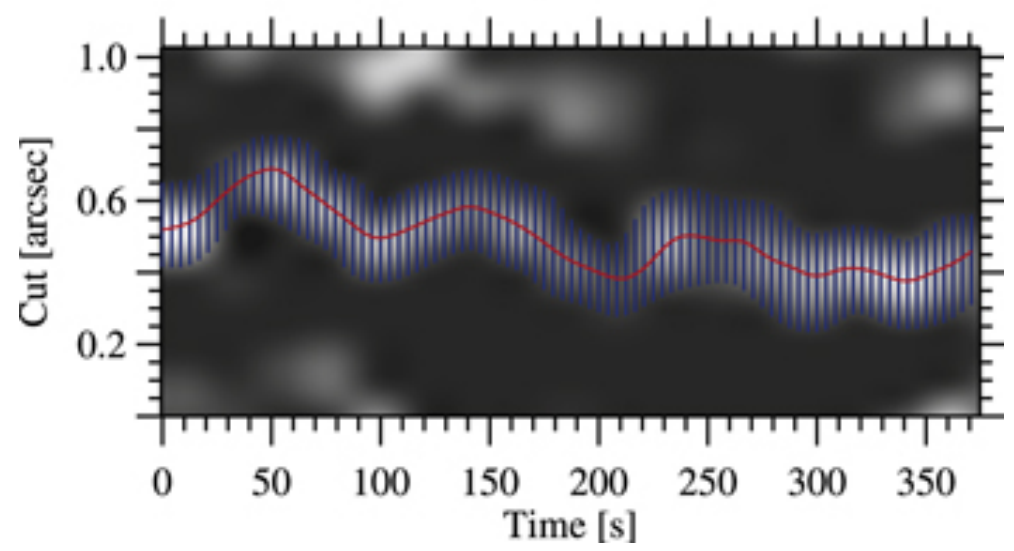

Figure A.4: Original (a) and restored (b) images of a slender Ca II H fibril (SCF) whose transverse oscillations along a cut perpendicular to its long axis is illustrated in panel (c). The yellow lines on panels (a) and (b) show the cut. Centroid of the transverse oscillation (the red line; smoothed by a box average of 0.05 arcsec width in space and $5 \mathrm{~s}$ width in time) and the width of the SCF (the blue vertical lines), determined using a semi-automated procedure (see the main text), are also depicted on the spacetime plot.

\section{A.3.3 Wave Analysis}

The majority of identified SCFs are uninterruptedly visible for a relatively short time, hence in most of the detected features the oscillatory behavior cannot be traced. Thus, wave propagation is investigated in only a small fraction of the oscillations identified in Section 3.2.2.

Propagation of transverse disturbances along a SCF can be deduced when oscillations are studied in at least two spatially separate cuts across the fibril axis. Propagation speeds in the SCF can then be estimated from phase differences between oscillatory motions at the different positions.

Figure A.5 shows an example of a relatively long-lived SCF (top panel) along with transverse oscillations (panels (a)-(e)) in the five cuts marked in yellow in the top panel (from left to right, respectively). We note that the cuts are chosen to lie relatively close to each other, where the SCF exists over the longest period of time (since the length of the SCF varies with time; see Gafeira et al. (2017b). The red triple-dot-dashed lines illustrate centroids of the oscillations. The green solid lines indicate linear fits to three selected extrema. These clearly demonstrate wave propagation in the SCF in one direction (i.e., from right to left along the SCF shown in the top panel). The transverse oscillations shown 
in Figure A.5 (a)-(e) include periods, transverse displacements, and velocity amplitudes in the range of $45-105 \mathrm{~s}, 15-70 \mathrm{~km}$, and $1.5-4 \mathrm{~km} \mathrm{~s}^{-1}$, respectively.

We use wavelet analysis (Daubechies 1990; Torrence and Compo 1998) to characterize wave propagation in the SCFs. In the method used here, which is described in detail by Jafarzadeh et al. (2017), the following steps are taken. (1) Spectral power of each transverse oscillation is simultaneously computed in the time and frequency domains. (2) Cross wavelet transforms, representing correlations between the wavelet power spectra of the oscillations at any two locations in a SCF (i.e., from the cuts across the fibrils) are determined. (3) Phase differences between each pair of oscillations whose correlated power spectra lie significantly above the noise are calculated. A significance level of 5\% is considered for the latter cross-power spectra.

Figure A.6 represents wavelet cross-power spectra between oscillations at pairs of locations (a-e) along the SCF shown in Figure A.5. The corresponding pairs of locations are indicated near the top of each panel. The power is represented by the background color, with yellow marking the highest power and gray the lowest. Regions with bleached color mark areas that are subject to the edge effect (the so-called cone of influence, COI). Thus, only values outside the COI are analyzed. Phase lags between oscillation pairs are depicted by the black arrows in selected locations. Arrows pointing right represent in-phase oscillations, i.e., $\varphi=0$, (and anti-phase oscillations when pointing left) and those pointing down indicate that the second oscillation leads the first one by $90^{\circ}$, i.e., a wave propagation from left to right in the SCF illustrated in the top panel of Figure A.5. The thick solid contours mark the 5\% significance level against red noise (increasing power with decreasing frequency), within which and outside the COI (i.e., in the region of consideration) the values of phase angles $(\varphi)$ along with their corresponding periods $(P)$ result in time differences $(\tau)$ that the waves need to travel between the pair of locations:

$$
\tau=\frac{\varphi P}{2 \pi}
$$

From the direction of the arrows in the regions of consideration in Figure A.6, a range of phase angles of the propagating waves in one direction (as was also noted from Figure A.5) are found in different times and locations in the sample SCF.

Knowing the distance between the cuts along which the oscillation pairs were measured as well as their corresponding time lags (computed from Equation A.4), we calculate phase speeds of the waves, representing the speed of wave propagation along a SCF (or a part of it). A median phase speed of $11 \pm 9 \mathrm{~km} \mathrm{~s}^{-1}$ was obtained for the waves propagating in the example SCF studied here (see Figure A.5 and Figure A.6. This large uncertainty in the propagation speeds obtained in a single SCF is likely due to the small distance between the individual cuts, so that even non-linearities in the wave-form or similar effects can result in rather different wave speeds. We note that the median phase speed determined from the wavelet analysis is in agreement with that calculated directly from shifts of the wave patterns in Figure 5. This sample SCF was found to have a median period, transverse displacement, and velocity amplitude of $64 \pm 14 \mathrm{~s}, 28 \pm 20 \mathrm{~km}$, and $1.8 \pm 1.1 \mathrm{~km} \mathrm{~s}^{-1}$, respectively.

While propagation in only one direction is the most common case in our sample (it is seen in $\approx 70 \%$ of the investigated SCFs), propagating waves in opposite directions are also observed in some of the SCFs under study. Figure A.7 and Figure A.8 represent one ex- 

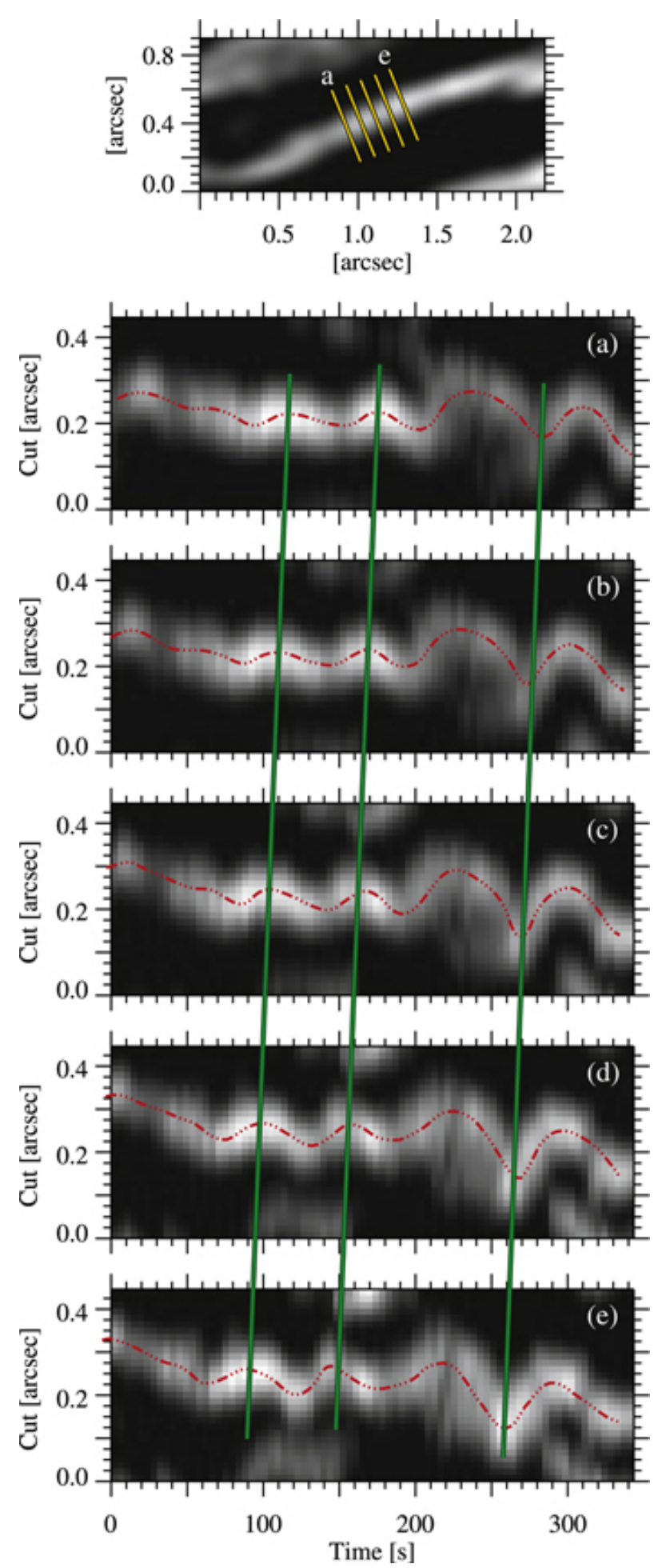

Figure A.5: Phase speeds in an example slender Ca II H fibril (SCF) with an indication of wave propagation. The curves in panels (a)-(e) represent displacement of the SCF along a series of cuts across the fibril shown in the upper panel, from left to right, respectively. The red triple-dot-dashed lines are the centroids of a Gaussian fit to the oscillations smoothed by convolving with a boxcar filter of 0.05 arcsec width. The green lines indicate waves propagation in the SCF in a direction corresponding to from right to left in the top panel. 

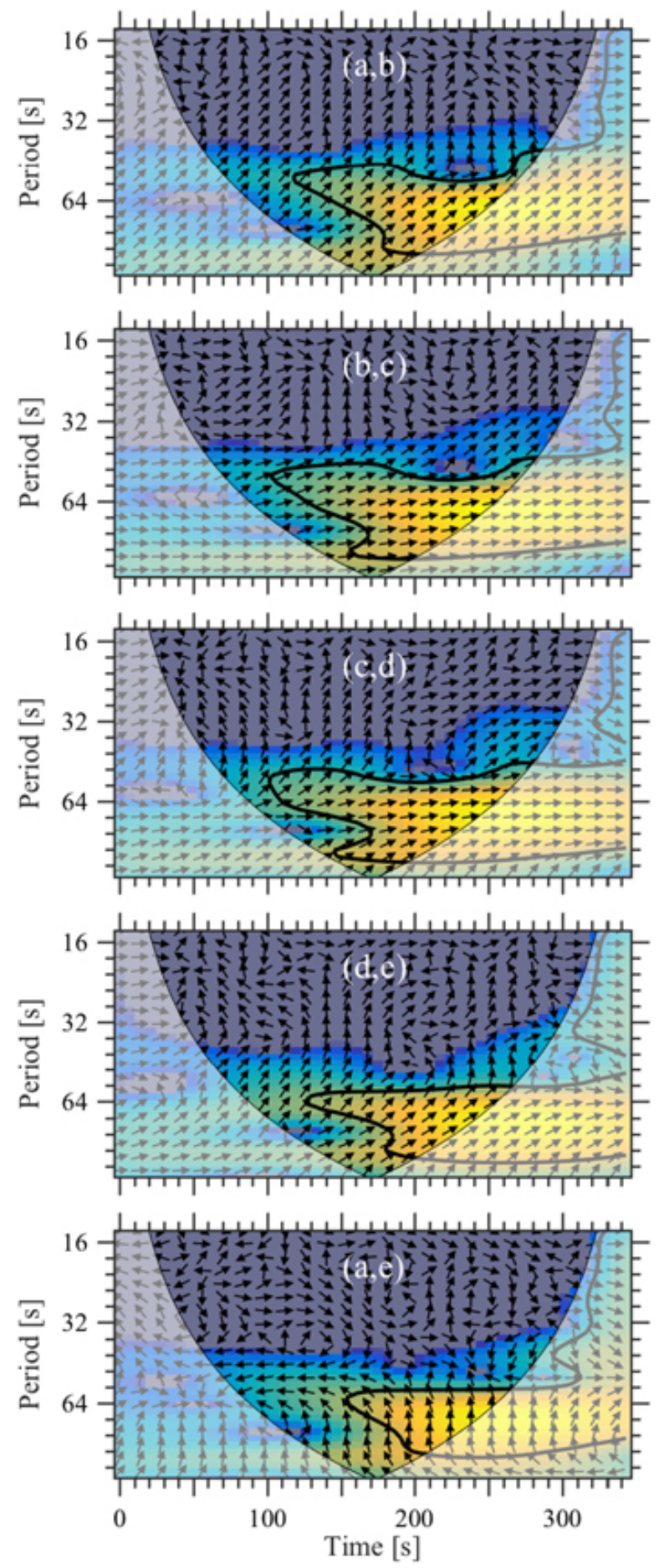

Figure A.6: Wavelet cross-power spectra between pairs of transverse perturbations shown in Figure A.5 (the pairs are indicated near the top of each panel). The background colors represent power spectra (with the highest power being colored yellow and the lowest power gray). The 5\% significance level against red noise is marked with a thick solid contour in each panel. The arrows depict the relative phase relationship between the pair of oscillations (with in-phase pointing right, anti-phase pointing left, and the second oscillation leading the first one by $90^{\circ}$ pointing straight down). The bleached areas indicate the cone of influence (see the main text). 
Table A.1: Properties of Transverse Oscillations/Waves in Slender Ca II H Fibrils (SCFs) Deduced from SunRISE/SUFI Data.

\begin{tabular}{lccccc}
\hline \hline Quantity & Range & Mean & Mode & Median & sigma $^{a}$ \\
\hline Period (s) & $16-199$ & 89 & 74 & 83 & 29 \\
Transverse displacement $(\mathrm{km})$ & $1-91$ & 35 & 26 & 33 & 16 \\
Transverse velocity $\left(\mathrm{km} \mathrm{s}^{-1}\right)$ & $1.0-4.8$ & 2.5 & 2.1 & 2.4 & 0.8 \\
Phase speed $\left(\mathrm{km} \mathrm{s}^{-1}\right)$ & $1-70$ & 15 & 5 & 9 & 14 \\
\hline
\end{tabular}

${ }^{a}$ Standard deviation of the distributions.

ample of such a complex wave propagation. The green lines in the former figure connect two selected extrema of the oscillations (along the five cuts shown in the top panel) and clearly demonstrate an oppositely propagating wave in the SCF: the left bump has a positive phase-lag, whereas the right dip has a negative phase relationship. From the direction of the arrows in the regions of consideration in Figure A.8, a range of phase angles of the propagating waves in opposite directions as well as in-phase/anti-phase oscillations are found in different times and locations in the sample SCF. The phase differences results in median propagating speeds of $15 \pm 12 \mathrm{~km} \mathrm{~s}^{-1}$ and $7 \pm 5 \mathrm{~km} \mathrm{~s}^{-1}$ for the oppositely moving waves in this SCF.

\section{A.3.4 Statistics}

We characterized transverse oscillations in a total of 134 SCFs observed with SUNRISE/SUFI. This includes measurements of their periods, transverse displacements, and velocity amplitudes (transverse velocities). Among all of these, it was possible to trace waves (i.e., characterize wave propagation) in only 23 relatively long-lived SCFs. Propagation speeds of the latter were then calculated from phase differences between oscillations detected along multiple cuts with known distances. We note that the SCFs under study were detected at different times over the $1 \mathrm{hr}$ time series of images. Table A.1 summarizes these properties, whose distributions are illustrated in Figure A.9. These include the periods, transverse displacements, and velocity amplitudes of the 134 independent SCFs, as well as the phase speed of the 23 long-lived features.

We note that individual disturbances often include a range of properties at their various spatial locations and/or at different times. Hence, in addition to different SCFs, the wide range of each quantity may also include the somewhat different properties of oscillations in individual SCFs (i.e., multiple periods observed in a SCF at different times). Thus, the distributions of period, transverse displacement, and velocity amplitude, shown in Figure A.9(a)-(c), include about 400 individual points. The histogram of the phase speed, although from 23 events, includes about 1400 data points from wavelet cross-power spectra of several pairs of transverse perturbations (the fluctuations were studied at several cuts perpendicular to the axis of each of the $23 \mathrm{SCFs}$ ).

Transverse oscillations in the SCFs are found to have a broad range of periods and phase speeds. These also include the propagation of waves in individual SCFs (see, e.g., Figure A.6 and Figure A.8 where the measured oscillations in a SCF have a wide range of periods and phase relationships). This suggests that the observed waves are incoherent 

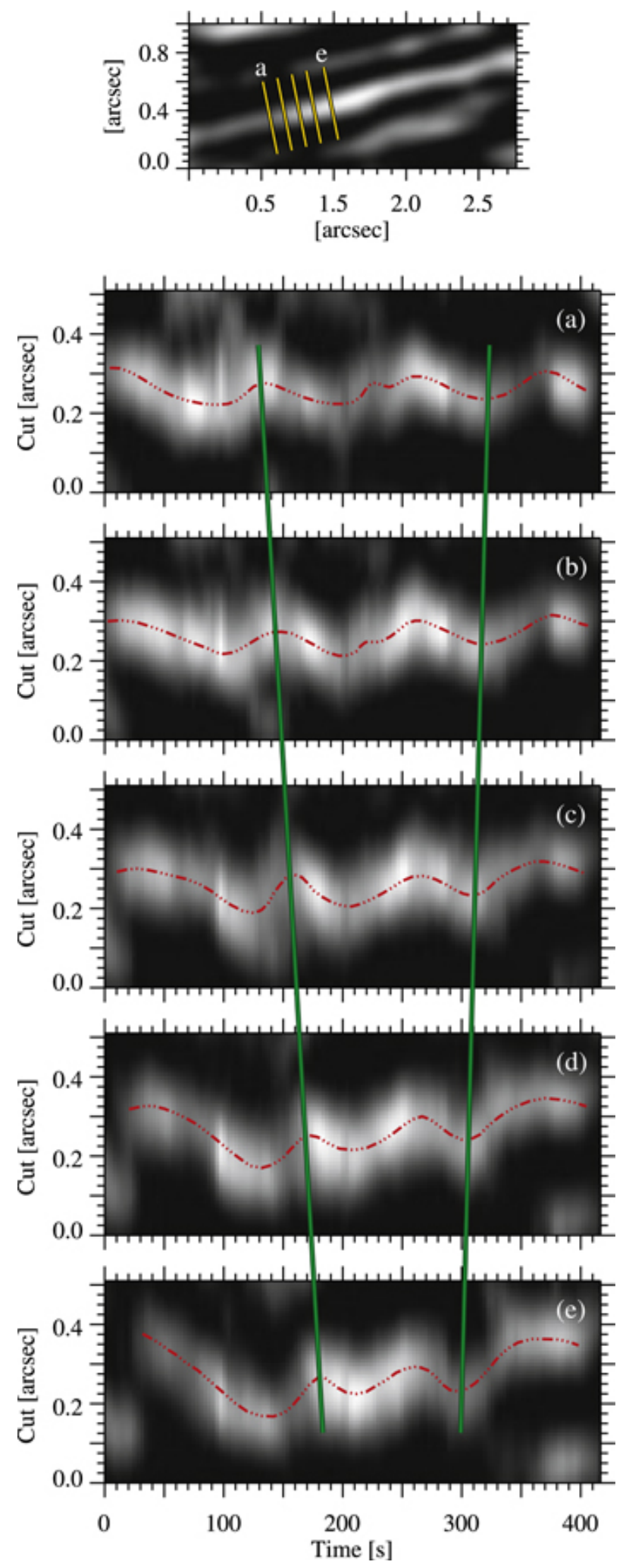

Figure A.7: Same as Figure A.5, but for a complex example. The green lines highlight waves in a SCF propagating in opposite directions. 

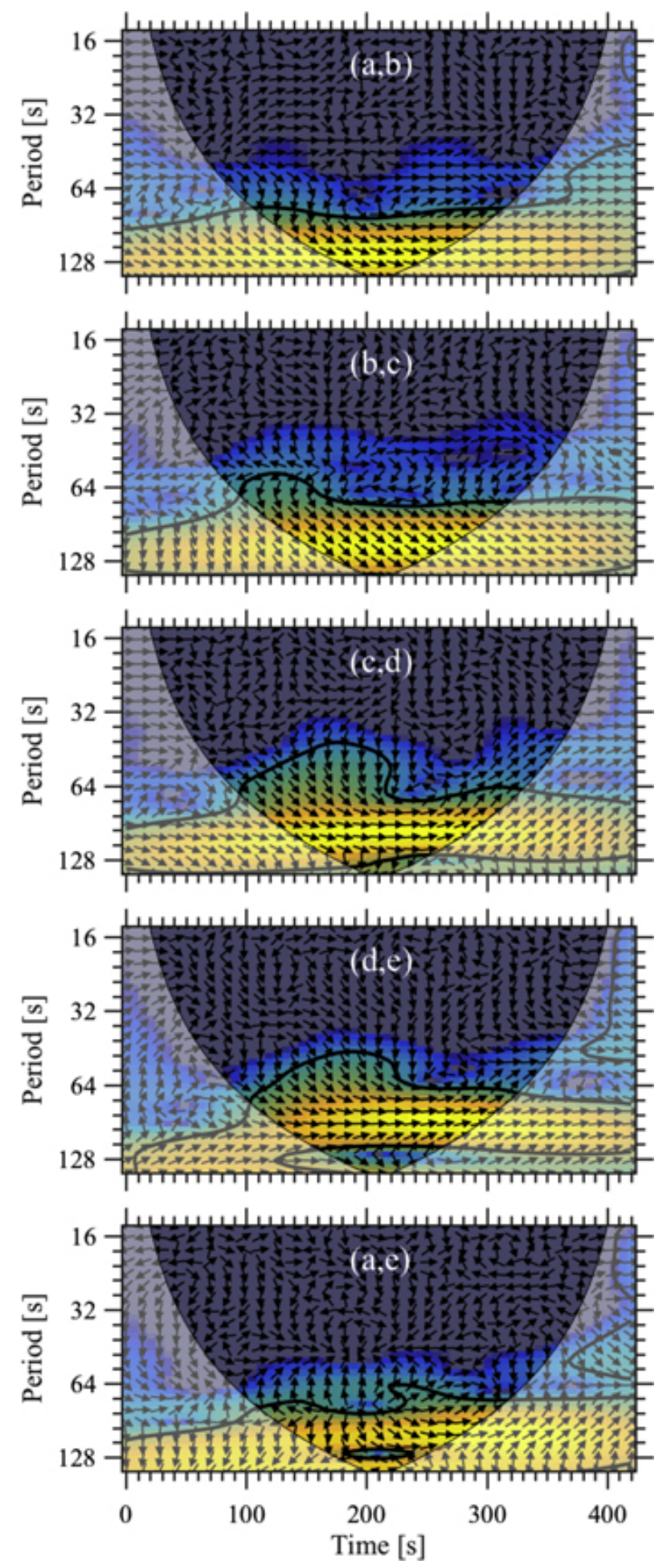

Figure A.8: Same as Figure A.6, but for transverse oscillations shown in Figure A.7. 

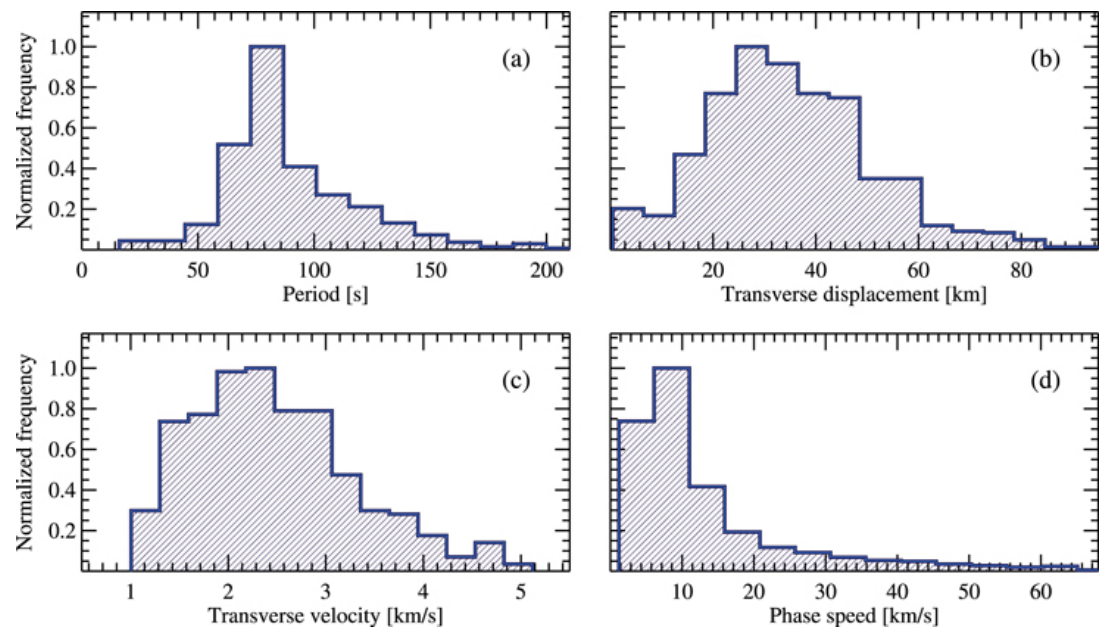

Figure A.9: Distributions of period (a), transverse displacement (b), transverse velocity (c), and phase speed (d) of oscillations in slender $\mathrm{Ca}$ II H fibrils. The histograms are normalized to their maximum values.

(i.e., each fibril oscillates independently).

\section{A.3.5 Energy Budget}

The energy flux transported by transverse waves can be estimated using

$$
E_{k}=\frac{1}{2} f\left(\rho_{i}+\rho_{e}\right) v^{2} c_{p h}
$$

if they are MHD kink waves (Van Doorsselaere et al. 2014). In Equation A.5, $\rho_{i}$ and $\rho_{e}$ are mass densities inside and outside the oscillating structure (here the SCFs), respectively, $v$ is the observed transverse velocity amplitude, $f$ is the density filling factor of discrete flux tubes, and $c_{p h}$ is the determined phase speed, respectively. The factor $1 / 2$ reflects the timeaveraged energy over one period. Obviously, misidentification of the wave mode (with a non-unity filling factor) results in over/underestimation of energy flux (Van Doorsselaere et al. 2008; Goossens et al. 2013).

Assuming that the SCFs represent flux tubes (which is not certain), the observed transverse oscillations, which are a result of the displacement of the SCFs' axes, can be interpreted as MHD kink waves (see Section 4 for further discussions).

By combining the observed velocity amplitude and phase speed of 2.4 and $9 \mathrm{~km} \mathrm{~s}^{-1}$, respectively, with a plasma mass density of $\rho_{i} \approx 10^{6} \mathrm{~kg} \mathrm{~m}^{3}$ (in the low chromosphere; at height $\approx 700 \mathrm{~km}$ from the visible surface) from a realistic radiative MHD simulation (Carlsson et al. 2016), we roughly estimate the energy flux of the kink waves in the SCFs to be $E_{k} \approx 15 \mathrm{~kW} \mathrm{~m}{ }^{2}$. We took $\rho_{i} / \rho_{e}=1$. Depending on whether the magnetic field is larger in the fibrils or outside them, this ratio will be less than or larger than unity. The energy flux for transverse waves will be smaller or larger depending on the ratio of densities between inside and outside the fibrils, but should, for reasonable assumptions of density contrast, not differ by more than a factor of three from the value deduced here. We approximated the filling factor of the SCFs to be $\approx 0.3$. This was determined from clean images where solar background, noise, and non-interesting brightenings were 
removed from the observed data (see Section 3.1). The ratio of the area filled by the SCFs to the total area (averaged over the entire time series of images) was taken as the filling factor. This would, however, be a lower limit for the filling factor, since many fibrils were excluded during the several steps of image restoration.

Also, we assume that the (magnetized) gas between the fibrils is not oscillating, which is unlikely, as the shaking fibril should also excite oscillations in that gas. The assumption of a kink wave requires that the density (and/or the magnetic field strength) inside the fibrils is different from that outside the fibrils. In the lower chromosphere, it is unclear to what extent such differences occur, or if the fibrils are mainly visible because of, e.g., a horizontal temperature inhomogeneity in the lower chromosphere leading to an inhomogeneous optical depth scale where the $\mathrm{Ca} \mathrm{II} \mathrm{H}_{2}$ peaks are formed.

\section{A.4 Discussion and Conclusions}

We have presented, for the first time to our knowledge, observations of transverse oscillations in SCFs. Seeing-free observations by the SUFI instrument onboard the SUNRISE $1 \mathrm{~m}$ solar telescope in a narrowband $\mathrm{Ca}$ II $\mathrm{H}$ filter have provided us with an excellent long time series of images sampling the lower solar chromosphere. The observed area was part of active region AR11768. The strongly enhanced Ca II H brightness in the ROI (see Figure 1) indicates a significant enhancement of the $\mathrm{H}_{2 V} / \mathrm{H}_{2 R}$ emission in the core of Ca II H (due to the enhanced temperatures in the presence of strong magnetic fields Solanki et al. 1991; Beck et al. 2013) so that the observed radiation contains significant contributions from the lower chromosphere (Jafarzadeh et al. 2017).

Our analysis of the SUNRISE/SUFI Ca II H data revealed that the transverse oscillations are ubiquitous in the lower solar chromosphere. To characterize the oscillations reliably, we limited our study to a relatively small number of isolated SCFs not affected by, e.g., the superposition of several SCFs and/or with other brightenings. Most SCFs are visible only for a short time at a stretch, are highly dynamic, and display a high number density in the studied area.

We found a wide range of periods, transverse displacements, velocity amplitudes, and phase speeds (when possible) for the oscillations/waves along the SCFs (see Figure A.9), with median values of $83 \mathrm{~s}, 33 \mathrm{~km}, 2.4 \mathrm{~km} \mathrm{~s}^{-1}$, and $9 \mathrm{~km} \mathrm{~s}^{-1}$, respectively (see Table A.1 for all statistical values).

The SCFs are found to share some physical characteristics with, e.g., type II spicules observed in the upper chromosphere according to Gafeira et al. (2017b). Table A.2 summarizes properties of transverse oscillations (and waves) for various fibrillar structures observed in different chromospheric passbands reported in the literature, along with our results for the SCFs (see Jess et al. 2015, for a detailed overview). 


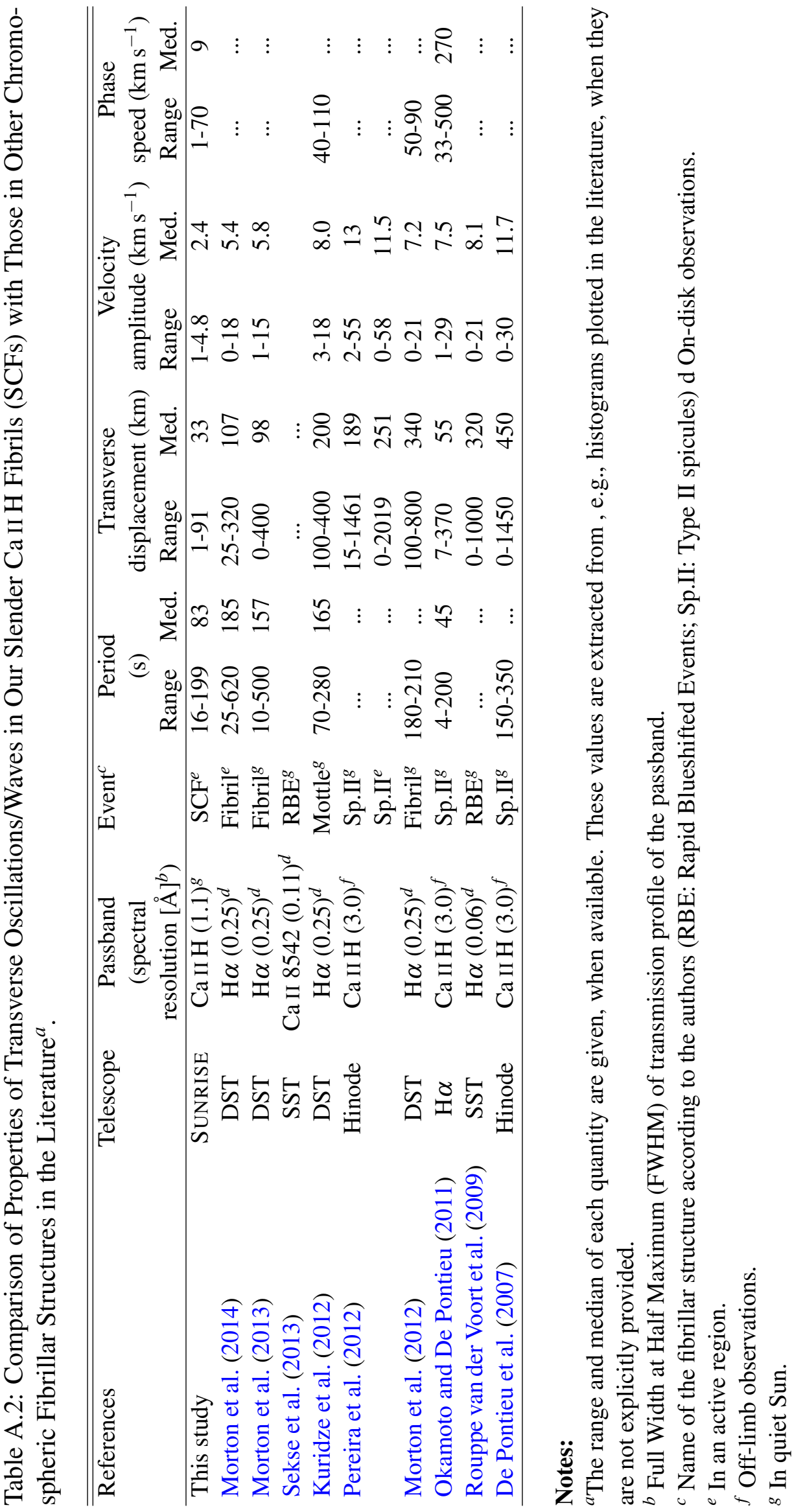


In addition to the ranges of different parameters of transverse oscillations in various chromospheric structures, we have also provided the median values of their distributions in Table A.2, when available. When not explicitly given, they were estimated from plotted distributions. We do not use mean values of the parameters, since they can be susceptible to the influence of outliers. The latter becomes particularly important in the case of fibrillar structures because their precise detection is often a challenge (or they could be mixed with other features of similar appearance). We stress that most of the other studies investigated waves on fibrillar structures located higher in the solar atmosphere (the off-limb studies even much higher), which might explain some of the differences seen in Table A.2 and discussed below.

The SCFs are found to have transverse oscillation with shorter periods than their counterparts in the higher chromospheric layers (with the median period of the former being shorter by a factor of $\approx 2$ than the latter). The median period of type II spicules reported by Okamoto and De Pontieu (2011) is, however, smaller than that of the SCFs (and other chromospheric fibrillar events, including type II spicules from the other studies). The authors explained their very short periods as a result of their method of analysis.

Transverse displacement, velocity amplitude and phase speed of the SCFs studied here are significantly smaller than those of the upper chromospheric elongated features (i.e., $\mathrm{H} \alpha$ fibrils, type II spicules, mottles and $\mathrm{H} \alpha / \mathrm{Ca}$ II $8542 \AA$ A RBEs). Transverse displacements of the relatively short-period type II spicules of Okamoto and De Pontieu (2011) are, however, comparable to (but slightly larger than) those we found for the SCFs (that is also likely to be an influence of their detection method). One reason for this discrepancy, even with structures observed by Hinode in the same spectral line is the fact that many of the other observations in $\mathrm{Ca}$ II $\mathrm{H}$ were off-limb, whereas we are observing near solar disk center. This makes the SUFI observations mainly sensitive to much lower levels in the solar atmosphere, where wave parameters, such as wave amplitudes, are expected to be lower due to the higher gas density, assuming the total energy flux in the waves to be the same at both layers. However, the higher spatial resolution of the SUFI data also allows smaller wave amplitudes to be detected.

The structures listed in Table A. 2 have been observed in various spectral lines in some cases off-limb so that they sample different heights in the solar chromosphere (Rutten 2007; Leenaarts et al. 2009, 2012). In the vigorously dynamic Sun even individual lines may obtain a contribution from a large range of heights (Rutten 2007). Hence, the lines used in the various observations may be affected by different local plasma properties, leading to subtle variations in the determined wave parameters (Jess and Verth 2015).

Hence, the chromospheric fibrillar structures sampled by the different observations can be of intrinsically different types (Rutten 2007), with more upright fibrils (potentially) channeling energy to the corona and more slanted ones transferring energy mainly within different parts of the chromosphere. The energies are likely generated in the photosphere as a result of the interactions between plasma and the magnetic field. Note that different characteristics of these lines with respect to opacity causes limb observations to represent much greater atmospheric heights compared to those on the disk. This is particularly the case for observations of type II spicules in $\mathrm{Ca}$ II $\mathrm{H}$ off the limb. In that observing geometry, the observed features lie in an upper chromospheric layer compared to on-disk observations of, e.g., SCFs, using a similar, or even narrower bandpass. These events may, however, share some apparent and/or physical characteristics (e.g., both types of 
underlying fibrils are observed as short-lived, dynamic, thin and bright elongated features in the Ca II H passband). Jafarzadeh et al. (2017a) showed that the SUNRISE/SUFI SCFs map the magnetic fields in the low solar chromosphere.

The observed transverse oscillations in the SCFs can clearly be described as waves propagating along the axes of the SCFs (when phase relationship between different locations along the fibril could be studied). In principle, both bulk Alfvén and kink waves can lead to transverse displacements. While some authors have interpreted transverse oscillations in chromospheric (and/or coronal) fibrillar structures, such as type II spicules, as Alfvénic waves (e.g., de Pontieu et al. 2007; Tomczyk et al. 2007; McIntosh et al. 2011), others have debated such interpretations and have explained them as a result of MHD kink waves (e.g., Van Doorsselaere et al. 2008, 2014; Goossens et al. 2013). In any case, they are Alfvénic, i.e., largely incompressible transverse oscillations propagating along the field lines, whose phase speed differs from the Alfvén speed only due to the inhomogeneity of the atmosphere (see, e.g., Priest 2014, for a detailed description of the terminology used here). The exact wave mode likely to be excited depends on the physical conditions in the SCFs and their surroundings, which are still largely unexplored. The bulk (shear) Alfvén waves only exist in a homogeneous medium (Nakariakov and Verwichte 2005; Goossens et al. 2013), that is likely not the case in the solar atmosphere.

The SCFs are possibly a manifestation of plasma jets along horizontal flux tubes, thus, according to the MHD wave theory, the kink mode is the most appropriate interpretation of their swaying motions (Kukhianidze et al. 2006; Erdélyi et al. 2007; Zaqarashvili et al. 2007). Note, however, that the fact that brightenings along the fibrils start more often inside the bulk of the fibrils than at one of their edges speaks against the interpretation of the fibrils as plasma jets. The incoherent oscillations among different SCFs disputes the option of global (bulk) Alfvén waves (Zaqarashvili and Erdélyi 2009, in addition to the assumption of the non-uniform medium), although it is unclear how large the coherence length must be to qualify as an Alfvén wave. A closer inspection would be necessary to confirm whether such incoherency is also consistent between neighboring SCFs. Note that incompressible, torsional Alfvén waves cannot displace flux tubes back and forth, hence they are unlikely to be responsible for the observed oscillations (Ploner and Solanki 1999; Erdélyi and Fedun 2007; Van Doorsselaere et al. 2008; Jess et al. 2009). The MHD kink waves can be triggered by granular buffeting of footpoints of magnetic flux tubes at the base of the photosphere (e.g., Spruit 1981; Choudhuri et al. 1993; Hasan and van Ballegooijen 2008) or by small-scale magnetic reconnection (He et al. 2009). The waves could also be generated as a result of wave coupling at plasma- $\beta=1$ regions (e.g., Bogdan et al. 2003; Roberts 2004).

Excursions in the upper chromospheric fibrillar structures (such as mottles, spicules, and $\mathrm{H} \alpha$ dynamic fibrils) have been suggested to be produced by chromospheric shock waves (De Pontieu et al. 2004; Hansteen et al. 2006). The chromospheric shocks are produced with different strengths and periods that consequently result in a wide range of observed properties of transverse oscillations (Hansteen et al. 2006). This mechanism could also be relevant for the origin of swaying motions in the SCFs, as shocks are also present in the lower chromosphere (e.g., Wedemeyer et al. 2004).

We note that the ROI, within which the SCFs were studied, was located outside pores, but in their immediate vicinity (see Figure A.1). Therefore, some of the waves in our SCFs, particularly close to the left and bottom edges of the ROI, could be excited 
within the pores, or could at least be influenced by pore waves (e.g., Morton et al. 2012; Khomenko and Collados 2015).

The observed high-frequency kink waves were estimated to carry an energy flux of order $15 \mathrm{~kW} \mathrm{~m}^{-2}$, which is comparable with what is needed to heat the (lower) solar chromosphere (Withbroe and Noyes 1977; Vernazza et al. 1981). Our observations cannot, however, clarify whether this energy is dissipated in the sampled atmospheric layers. The apparently almost horizontal orientation of the majority of the SCFs studied here, many of which connect back to the solar surface as low-lying loops (Jafarzadeh et al. 2017, see), suggest that the waves propagating along them may not reach the upper atmosphere. Rather, they play a role in heating their local surroundings, if they release their energy at these layers. Jafarzadeh et al. (2017) showed with the help of the magnetostatic solution of Wiegelmann et al. (2017) that the shorter SCFs map fields that are more vertical. A number of the transverse waves we have identified are found along these short fibrils. These waves are likely capable of traveling upwards into the upper atmosphere and may even reach to the corona, contributing to the heating there.

To determine which mechanism can precisely explain the nature of bright and highly dynamic SCFs, analysis of high-resolution observations in a narrower $\mathrm{Ca}$ II H passband (e.g., from the upcoming SST/CHROMIS instrument) as well as realistic MHD simulations of this line will be necessary. 



\section{Publications}

\section{Refereed publications}

- R. Gafeira, A. Lagg, S. K. Solanki, S. Jafarzadeh, M. van Noort, P. Barthol, J. Blanco Rodríguez, J. C. del Toro Iniesta, A. Gandorfer, L. Gizon, J. Hirzberger, M. Knölker, D. Orozco Suárez, T. L. Riethmüller, and W. Schmidt, published in ApJS, 229, 2017, DOI 10.3847/1538-4365/229/1/6

- R. Gafeira,S. Jafarzadeh,S. K. Solanki, A. Lagg, M. van Noort, P. Barthol, J. Blanco Rodríguez, J. C. del Toro Iniesta, A. Gandorfer, L. Gizon, J. Hirzberger, M. Knölker, D. Orozco Suárez, T. L. Riethmüller, and W. Schmidt, published in ApJS, 229, 2017, DOI 10.3847/1538-4365/229/1/7

- S. Jafarzadeh, S. K. Solanki, R. Gafeira, M. van Noort, P. Barthol, J. Blanco Rodríguez, J. C. del Toro Iniesta, A. Gandorfer, L. Gizon, J. Hirzberger, M. Knölker, D. Orozco Suárez, T. L. Riethmüller, and W. Schmidt published in ApJS, 229, 2017, DOI 10.3847/1538-4365/229/1/9. 



\section{Acknowledgements}

My first thanks are going to my supervisors Sami Solanki and Andreas Lagg. Thanks to Sami, for all his guidance, interesting discussions and support. Big thanks to Andreas for all his help and patience during the progress of my work, and infinite help in the process of writing the papers and thesis. Your help and our end of afternoon beneficial, efficient, extensive, refreshing meetings were essential to me to be able to finish my thesis. Thanks to Laurent for the valuable output and carefully reading my thesis.

Obrigado aos meus pais, Francisco e Graça e ao meu irmão Tiago e aos meus avos estarem sempre presentes e por serem a melhor família que alguém pode ter.

Thanks for all the good moments, and for the support and help to overpass the difficult times to Filipa, Francisco Iglesias, David, Ivan, Alessandro, Michel, Azaymi, Iulia, Suzana, Isabel, and Pedro Lacerda that I have the luck to meet during this journey that I have the privilege to call friends.

Thanks to the ones who made my stay in Göttingen much nicer. Borys, Sabrina, Sihane, Marta, Gonçalo, Hans-Peter, Iker, Benjamin, Cátia, Belisa, Rita, Francisco, Luis, and so many others. Thanks to my old friends that even far away they were always present for me, Sara, Joana, Carina, Ricardo Vieira, Fabio, Tiago, Teresa, Peixinho, Sr. Claudino, Dr. Adriana, Dr. JoÃčo Fernandes.

Thanks to Shahin for all his help during the thesis and support during my visit to Institute of Theoretical Astrophysics in Oslo. To my officemates Mariangela, Patricio, Fabian, Chi Ju, and Farhad. To all SLAM members, in particular to Michiel, for the help and availability to always answer to my question.

Thanks to the International Max Planck Research School (IMPRS) for Solar System Science at the University of Göttingen for the financial support and to the school coordinator Sonja Schuh for all the help.

This thesis is dedicated to my parents and my brother. 



\title{
Curriculum vitae
}

\author{
Ricardo Jorge Maranhas Gafeira
}

Born in August 1987 in Guarda, Portugal

Portuguese

email:gafeira@gmail.com

\section{Education}

- 2014-today, PhD. Student, Max Planck Institute for Solar System Research and University of Göttingen, Göttingen, Germany

- 2009-2011, M. Sc. in Astrophysics and Space Instrumentation, Faculty of Sciences and Technology of University of Coimbra, Portugal

- 2005-2009, B. Sc. in Physics with a Space Sciences Minor, Faculty of Sciences and Technology of University of Coimbra, Portugal

\section{Selected oral presentations}

- Small scale chromospheric fibrils observed by Sunrise II, R. Gafeira, A. Lagg, M. van Noort, S. K. Solanki, 4th SOLARNET meeting "The physics of the Sun from the interior to the outer atmosphere", 20-23 June of 2016, Arrecife, Lanzarote,Spain

- Small scale chromospheric fibrils observed by Sunrise II, R. Gafeira, A. Lagg, M. van Noort, S. K. Solanki, IRIS-6: The chromosphere, 16-20 January of 2016, University of Stockholm,Stockholm,Sweden

- Temporal variations in small scale chromospheric fibrils observed by Sunrise II, R. Gafeira, A. Lagg, M. van Noort, S. K. Solanki, Ground based Solar Observations in the Space Instrumentation Era, 05-09 October of 2015, University of Coimbra, Coimbra, Portugal

- Variation rate of sunspot area, R. Gafeira, A.Pais, C. Fonte, J. Fernandes,XXII National meeting of Astronomy and Astrophysics, 23-25 September of 2012, University of Porto, Porto, Portugal 


\section{Poster communication}

- Temporal variations in small scale chromospheric fibrils observed by Sunrise, R. Gafeira, A. Lagg, M. van Noort, S. K. Solanki, Annual Meeting of the Astronomische Gesellschaft, 22-26 September of 2014, University of Bamberg, Bamberg, Germany

- Variation rate of sunspot area, R. Gafeira, A.Pais, C. Fonte, J. Fernandes, European Week of Astronomy and Space Science, 1-6 July, Pontificia Università Lateranense, Rome, Italy

- Solar filaments : Characteristics and Evolution , R. Gafeira, J. Fernandes, J. Aboudarham, XXI National meeting of Astronomy and Astrophysics 7-10 September of 2011, University of Coimbra, Coimbra, Portugal

\section{Book Chapters}

- Claudio Paulo, Joana Latas, Leonor Cabral, Lina Canas, Lucio Carvalho, Luis Cardoso, Manuel Penhor, Paula Furtado, Paulo Jorge Lourenco, Ricardo Gafeira, Rita Guerra, Rosa Doran, "Do Planeta Terra ao Espaco - Sugestoes de tarefas experimentais para dentro e fora da sala de aula", ISBN: 978-989-20-5053-9

\section{Publications}

- R. Gafeira, A. Lagg, S. K. Solanki, S. Jafarzadeh, M. van Noort, P. Barthol, J. Blanco Rodríguez, J. C. del Toro Iniesta, A. Gandorfer, L. Gizon, J. Hirzberger, M. Knölker, D. Orozco Suárez, T. L. Riethmüller, and W. Schmidt. Morphological Properties of Slender Ca II H Fibrils Observed by Sunrise II. The Astrophysical Journal Supplement Series, 229:6, March 2017

- R. Gafeira, A. Lagg, S. K. Solanki, S. Jafarzadeh, M. van Noort, P. Barthol, J. Blanco Rodríguez, J. C. del Toro Iniesta, A. Gandorfer, L. Gizon, J. Hirzberger, M. Knölker, D. Orozco Suárez, T. L. Riethmüller, and W. Schmidt. Morphological Properties of Slender Ca II H Fibrils Observed by Sunrise II. The Astrophysical Journal Supplement Series, 229:6, March 2017

- R. Gafeira, S. Jafarzadeh, S. K. Solanki, A. Lagg, M. van Noort, P. Barthol, J. Blanco Rodríguez, J. C. del Toro Iniesta, A. Gandorfer, L. Gizon, J. Hirzberger, M. Knölker, D. Orozco Suárez, T. L. Riethmüller, and W. Schmidt. Oscillations on Width and Intensity of Slender Ca II H Fibrils from Sunrise/SuFI. The Astrophysical Journal Supplement Series, 229:7, March 2017

- S. Jafarzadeh, S. K. Solanki, R. Gafeira, M. van Noort, P. Barthol, J. Blanco Rodríguez, J. C. del Toro Iniesta, A. Gandorfer, L. Gizon, J. Hirzberger, M. Knölker, D. Orozco Suárez, T. L. Riethmüller, and W. Schmidt. Transverse Oscillations in Slender Ca II H Fibrils Observed with Sunrise/SuFI. The Astrophysical Journal Supplement Series, 229:9, March 2017. 
- S. Carvalho, P. Pina, T. Barata, R. Gafeira, and A. Garcia. Ground-based Observations of Sunspots from the Observatory of Coimbra: Evaluation of Different Automated Approaches to Analyse its Datasets. In I. Dorotovic, C. E. Fischer, and M. Temmer, editors, Coimbra Solar Physics Meeting: Ground-based Solar Observations in the Space Instrumentation Era, volume 504 of Astronomical Society of the Pacific Conference Series, page 125, April 2016

- R Gafeira, CC Fonte, MA Pais, and J Fernandes. Temporal evolution of sunspot areas and estimation of related plasma flows. Solar Physics, 289(5):1531-1542, 2014

- Gafeira, R., Patacas, C., and Fernandes, J. Mass-luminosity relation for fgk main sequence stars: metallicity and age contributions. Astrophysics and Space Science, 341:405-410, 2012

- Peixinho, N., Delsanti, A., Guilbert-Lepoutre, A., Gafeira, R., and Lacerda, P. The bimodal colors of centaurs and small kuiper belt objects. A\&A, 546:A86, 2012 